\title{
Quantum Knots and Lattices, or a Blueprint for Quantum Systems that Do Rope Tricks
}

\author{
Samuel J. Lomonaco and Louis H. Kauffman
}

\begin{abstract}
Within the framework of the cubic honeycomb (cubic tessellation) of Euclidean 3-space, we define a quantum system whose states, called quantum knots, represent a closed knotted piece of rope, i.e., represent the particular spatial configuration of a knot tied in a rope in 3-space. This quantum system, called a quantum knot system, is physically implementable in the same sense as Shor's quantum factoring algorithm is implementable.

To define a quantum knot system, we replace the standard three Reidemeister knot moves with an equivalent set of three moves, called respectively wiggle, wag, and tug, so named because they mimic how a dog might wag its tail. We argue that these moves are in fact more "physics friendly" than the Reidemeister moves because, unlike the Reidemeister moves, they respect the differential geometry of 3-space, and moreover they can be transformed into infinitesimal moves.

These three moves wiggle, wag, and tug generate a unitary group, called the lattice ambient group, which acts on the state space of the quantum system. The lattice ambient group represents all possible ways of moving a rope around in 3-space without cutting the rope, and without letting the rope pass through itself.

We then investigate those quantum observables of the quantum knot system which are knot invariants. We also study Hamiltonians associated with the generators of the lattice ambient group. We conclude with a list of open questions.
\end{abstract}

\section{Contents}

1. Introduction 210

2. Part 0. The quest for a more "physics-friendly" set of knot moves 211

3. How does a dog wag its tail? 211

4. Clues from mechanical engineering 212

5. A translation of mechanical engineering into knot theory 216

6. Part 1: Lattice Knots 218

2010 Mathematics Subject Classification. Primary 81P68, 57M25, 81P15, 81P40, 81P45, 68Q12, 57M27; Secondary 20C35.

Key words and phrases. Quantum Knots, Knots, Knot Theory, Quantum Computation, Quantum Algorithms, Quantum Entanglement, Knot Invariants, Quantum Measurement, Schroedinger's Equation, Hamiltomian, Calculus of Variation, Variational Derivatives.

(C)2010 American Mathematical Society 
7. Lattice knots 218

8. Basic terminology and conventions 219

9. Lattice Knot Moves: Wiggle, Wag, and Tug 224

10. The ambient groups $\Lambda_{\ell}$ and $\widetilde{\Lambda}_{\ell} \quad 232$

11. Conditional auto-homeomorphism representations of $\Lambda_{\ell} \quad 236$

12. The refinement injection and the conjectured refinement morphism 242

13. Lattice knot type 243

14. The preferred vertex (PV) approximation for knots 243

15. Wiggle, Wag, and Tug variational derivatives, and knot invariants 246

16. Infinitesimal Wiggles, Wags, and Tugs, differential forms, and integrals 249

17. n-bounded lattice knots 249

18. Part 2. Quantum Knots 251

19. The Definition of a Quantum Knot 252

20. Quantum knot type 254

21. Hamiltonians of the generators of the ambient group $\Lambda$. 255

22. Quantum observables as invariants of quantum knots 257

23. Conclusion: Open questions and future directions 261

24. Appendix A: A quick review of knot theory 265

25. Appendix B: A Rosetta Stone for notation 267

26. Appendix C: The refinement morphism conjecture 268

27. Appendix D: Oriented quantum knots 273

28. Appendix E: Quantum graphs 274

$\begin{array}{ll}\text { References } & 274\end{array}$

\section{Introduction}

Throughout this paper, the term "knot" means either a knot or a link. For those unfamiliar with knot theory, we refer them to a quick overview of the subject given in appendix $\mathrm{A}$.

This paper is a sequel to the research program on quantum knots begun and defined in [25]. This sequel is motivated by the difficulties encountered by the first author in applying knot theory to physics while writing the paper [26] on classical electromagnetic knots.

The key difficulty encountered in writing [26] is that physics "lives" in geometric space and, on the other hand, knot theory "lives" in topological space. As a consequence, in knot theory the inherent geometric structure of 3-space is often ignored, or simply discarded. If one's objective is to solve the central problem of knot theory, i.e., the placement problem, then it is a sound strategy frequently to ignore the unneeded non-pertinent geometric structure of 3-space. However, if one's objective is to use knot theory as a tool for investigating problems in physics, then this may not be the best strategy.

Case in point is the set of the Reidemeister moves. These moves have become one of the major corner stones of knot theory. They are two dimensional moves which ignore much of the geometry that is naturally a part of geometric 3-space. 
They do so by focusing on the planar projections of knots. For example, the Reidemeister moves inherently depend on the concept of a knot crossing. However, knots do not have crossings! After all, a knot crossing is simply a "figment" of one's chosen projection.

What is needed for applications to physics is another set of moves that is more sensitive to the inherent differential geometry of 3-space. For that reason (among others), we will introduce as a possible alternative to the three Reidemeister moves, three moves called wiggle, wag, and tug.

\section{Part 0. The quest for a more "physics-friendly" set of knot moves}

However, before we can define the three moves, wiggle, wag, and tug, we first need to gain a better understanding of how a dog wags it tail.

\section{How does a dog wag its tail?}

The first author's best friend Tazi certainly knew how to wag her tail.

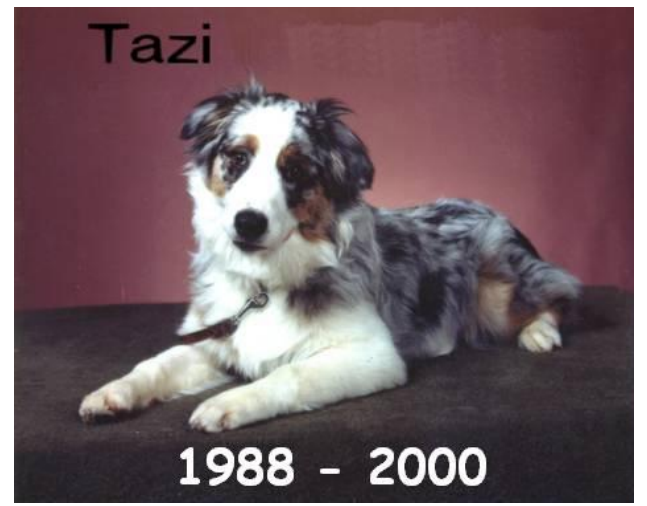

The first author's best friend Tazi knew the answer.

She would wiggle her tail, much as a creature would squirm on a flat planar surface, such as for example:

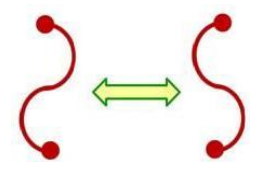

She would wag her tail in a twisting corkscrew motion, such as for example:

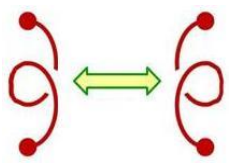

Her tail would also stretch or contract when an impolite child would tug on it, such as for example: 


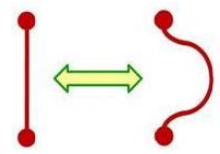

Yes, when Tazi moved her tail, she naturally understood how a curve can move in 3-space. She had a keen understanding of the differential geometry of curves. She instinctively understood that each point of a (sufficiently well behaved) curve in 3-space naturally has associated with it a 3 -frame, called the Frenet frame ${ }^{1}$, consisting of the unit tangent vector $T$, the unit normal vector $N$, and the unit binormal vector $B$. She instinctively understood that

- A curve instantaneously bends by rotating about its binormal $B$, as measured by its curvature $\kappa$,

- A curve instantaneously twists by rotating about its normal $N$, as measured by it torsion $\tau$, and

- A curve instantaneously stretches or contracts along its tangent $T$.

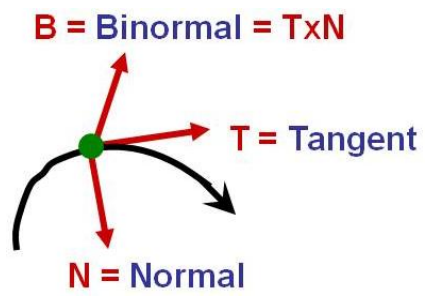

The Frenet Frame.

Key Intuitive Idea: A sufficiently well-behaved curve in 3-space has at each point three infinitesimal degrees of freedom.

Tazi understood the key intuitive idea that a curve in 3-space has at each point three local (i.e., infinitesimal) degrees of freedom. Can we use this intuition to create a usable well-defined set of moves which can form a basis for knot theory, much as the Reidemeister moves have filled that role?

\section{Clues from mechanical engineering}

Question: Can we transform this intuition into a mathematically rigorous definition? In particular, can we transform the following intuitive moves into well-defined infinitesimal moves?

\footnotetext{
${ }^{1}$ For readers unfamiliar with differential geometry, please refer to, for example, $[\mathbf{9}, \mathbf{3 6}, \mathbf{4 9}]$.
} 

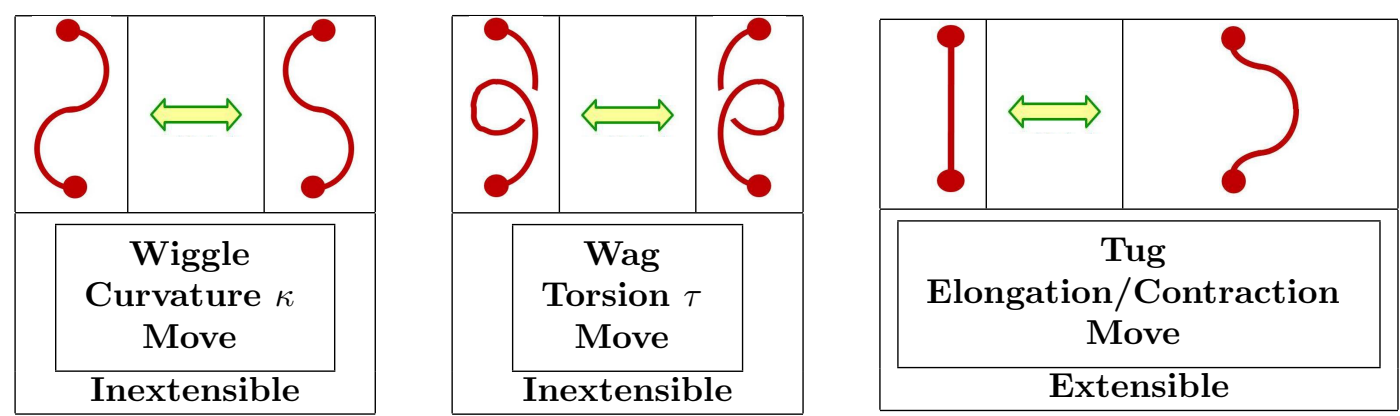

There are clues from mechanical engineering that suggest a possible approach to creating a mathematically rigorous definition.

In mechanical engineering, a linkage is a sequence of inextensible bars (i.e., rods) connected by joints

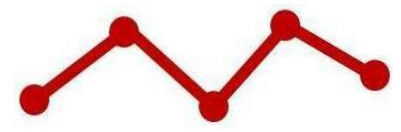

\section{A mechanical linkage.}

We will have need of the following three kinds of mechanical joints, planar (a.k.a. revolute, pin, or hinge), spherical (a.k.a., ball or socket), and prismatic (a.k.a. slider), which are illustrated below:

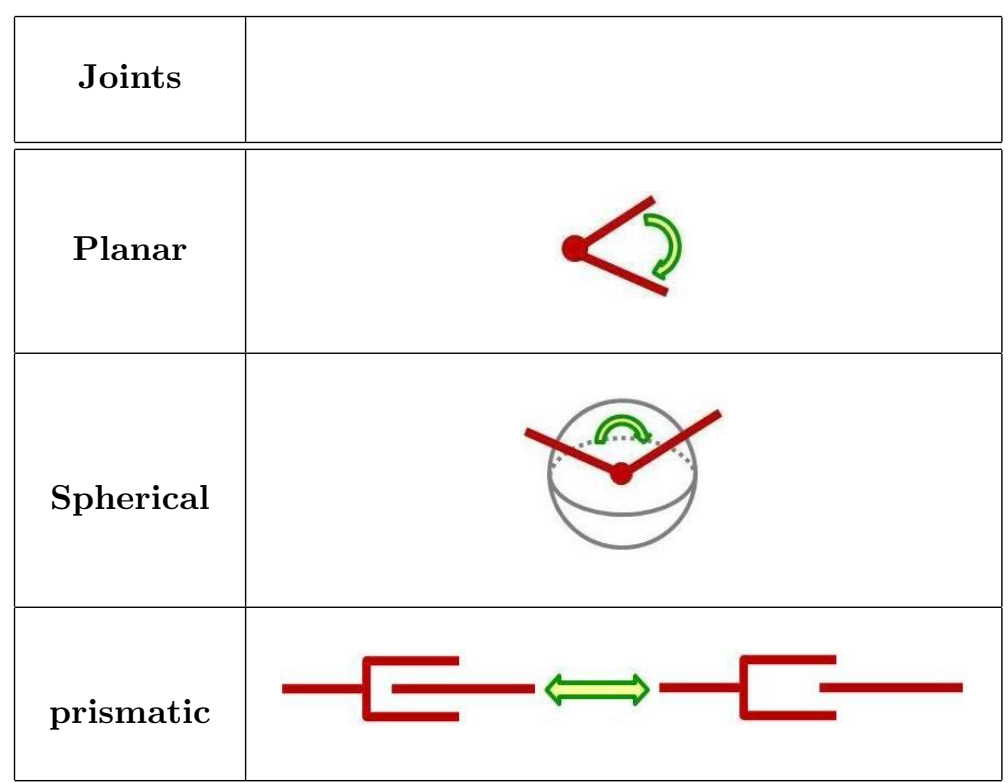

In mechanical engineering, a mechanism is a linkage with one degree of freedom. We will consider the following three mechanisms:

- The 4-Bar Linkage: The 4-bar linkage, illustrated below, has exactly one degree of freedom. 


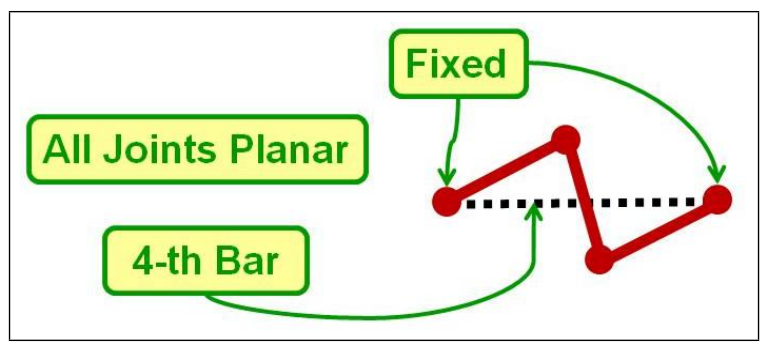

All joints in this linkage are planar. Since the leftmost and rightmost joints are fixed (stationary), the missing fourth bar is effectively the dotted line shown in the figure. If the leftmost and the rightmost joints are connected to other linkages, then movement of the 4-bar linkage does not effect any bars of the larger composite linkage other than the above three red bars. In other words, the 4-bar linkage is a local move on a larger linkage.

In particular, the 4-bar linkage gives an illustration of a local curvature move, taking place in a plane. We will call this local move a wiggle.

- The 3-Bar Linkage: The 3-bar linkage, illustrated below, has one degree of freedom.

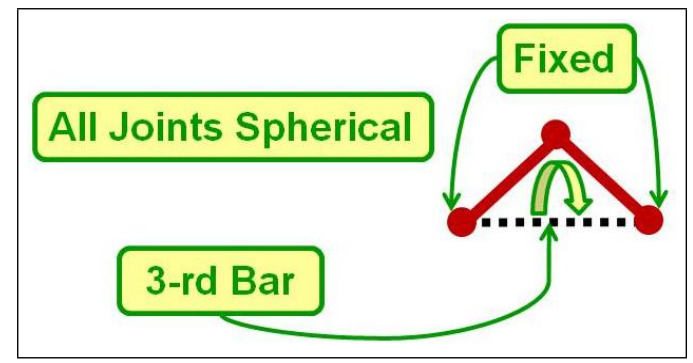

All joints in this linkage are spherical. Since its outermost joints are fixed (stationary), the missing third bar is effectively the dotted line shown in the figure. If the outermost joints are connected to other linkages, then movement of the 3-bar linkage does not effect any bars of the larger composite linkage other than the above two red bars. In other words, the 3 -bar linkage is a local move on a larger linkage.

This is a local torsion move, locally twisting a portion of the linkage into a new plane. We call it a wag.

- The 4-Bar Slider: The 4-bar slider, illustrated below, has one degree of freedom. 


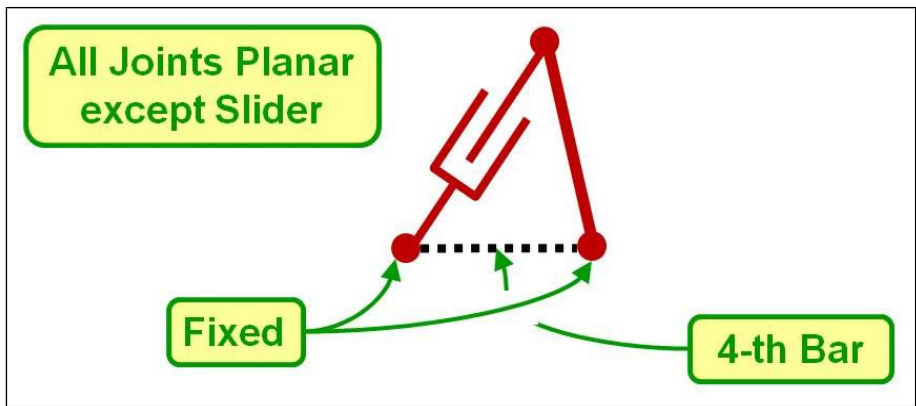

All joints in this linkage are planar except for the prismatic joint. Since the outermost joints are fixed (stationary), the missing fourth bar is effectively the dotted line shown in the figure. If the outermost joints are connected to other linkages, then movement of the 4-bar slider does not effect any bars of the larger composite linkage other than the above three red bars. Thus, the 4-bar slider can be thought of as a local move on a larger linkage.

This is a local expansion/contraction move, taking place in a fixed plane. We will call it a tug.

Before closing this section, we should mention three striking examples of linkages. The first is the Tangle [51], invented by Richard E. Zawitz, and shown in the figure given below:

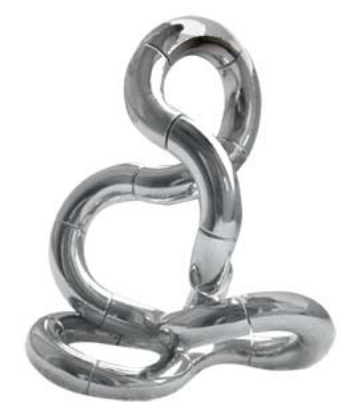

\section{Zawitz's Tangle ${ }^{\circledR}$ moves only by wagging.}

The Tangle is a linkage with only one local degree of freedom. It moves only by wagging.

The second and third linkages are the Bendandle ${ }^{\mathrm{TM}}$ and the Universal Bendangle $^{\mathrm{TM}}$, invented by Samuel J. Lomonaco (patents pending), and shown respectively in the two figures given below: 


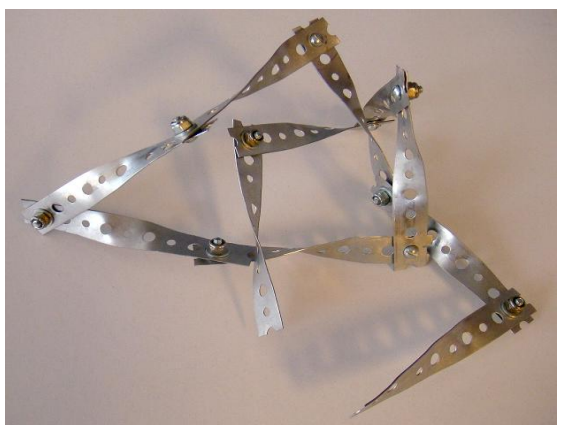

Lomonaco's Bendangle ${ }^{\mathrm{TM}}$ moves only by wiggling. (Patent pending)

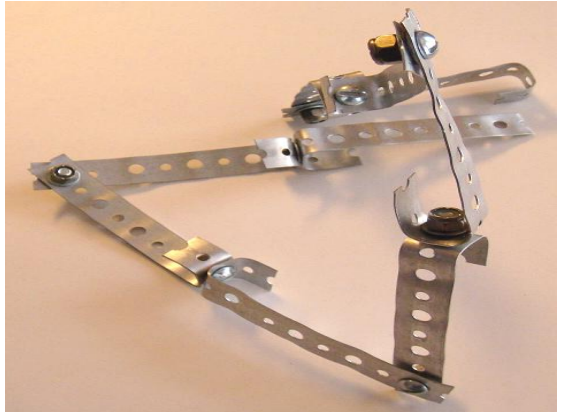

Lomonaco's Universal Bendangle $^{\text {TM }}$ moves only by wiggling and wagging. (Patent pending.)

The Bendangle also has only one local degree of freedom, but in this case, moves only by bending. The Universal Bendangle, as its name suggests, has two local degrees of freedom, moving only by bending and twisting.

In some sense, these three examples further support the key intuition that curves in 3 -space have in some sense three local degrees of freedom.

\section{A translation of mechanical engineering into knot theory}

Let us now translate mechanical engineering into knot theory.

Definition 5.1. Two piecewise linear (PL) knots $K_{1}$ and $K_{2}$ are said to be of the same knot type, written

$$
K_{1} \sim K_{2}
$$

provided there exist finite subdivisions $K_{1}^{\prime}$ and $K_{2}^{\prime}$ of $K_{1}$ and $K_{2}$ respectively such that one can be transformed into the other by a finite sequence of the following three local moves:

1) A tug:

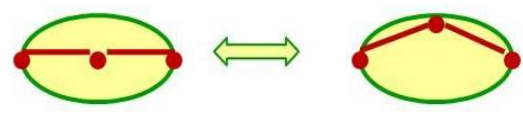

2) A wiggle:

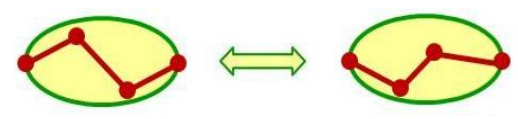


3) A wag:

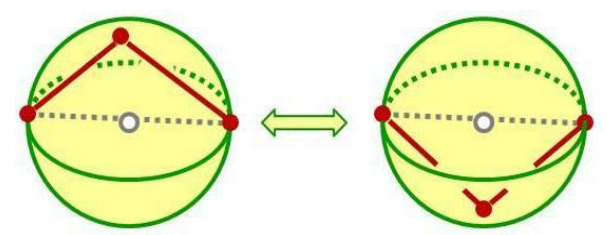

Using the methods found in Reidemeister's proof of the completeness of the Reidemeister moves, we have:

THEOREM 5.2. Wiggles and wags can be expressed as finite sequences of tugs.

REMARK 5.3. In fact, Reidemeister's fundamental move, i.e., his triangle move, is essentially a tug. (See $[\mathbf{4 2}, \mathbf{4 3}]$.)

So it would appear that we have accomplished nothing!

But on the contrary, we have indeed accomplished something after all. For we are now in a position to alter knot theory in such a way as to incorporate more of the geometry of 3 -space. The telltale clue is that wiggle and wag are inextensible moves, while tug is not. By an inextensible move, we mean one that does not locally change the length of a curve (and hence, preserves global length.)

Definition 5.4. Two piecewise linear (PL) knots $K_{1}$ and $K_{2}$ are said to be of the same inextensible knot type, written

$$
K_{1} \approx K_{2}
$$

provided there exist finite subdivisions $K_{1}^{\prime}$ and $K_{2}^{\prime}$ of $K_{1}$ and $K_{2}$ respectively such that one can be transformed into the other by applying a finite sequence of wiggles and wags.

Theorem 5.5. Two PL knots $K_{1}$ and $K_{2}$ are of the same knot type if and only if they have

1) The same inextensible knot type, i.e. $K_{1} \approx K_{2}$, and

2) The same length, i.e., $\left|K_{1}\right|=\left|K_{2}\right|$.

Thus, nothing from classical knot theory is lost with the above modified definition of knot type. But on the other hand, with this modified definition, we have succeeded in incorporating more of the geometry of 3 -space into knot theory! ${ }^{2}$

\footnotetext{
${ }^{2}$ For a more detailed justification of this definition, we refer the reader to Section 16 of this paper.
}

This is a free offprint provided to the author by the publisher. Copyright restrictions may apply. 


\section{Part 1: Lattice Knots}

Lest we forget, one of our objectives is to create a firm mathematical foundation for the intuition that sufficiently well-behaved curves in 3-space have three local (i.e., infinitesimal) degrees of freedom. We would like to answer the following question:

Question: Can we transform the following intuitive moves into well-defined infinitesimal moves?
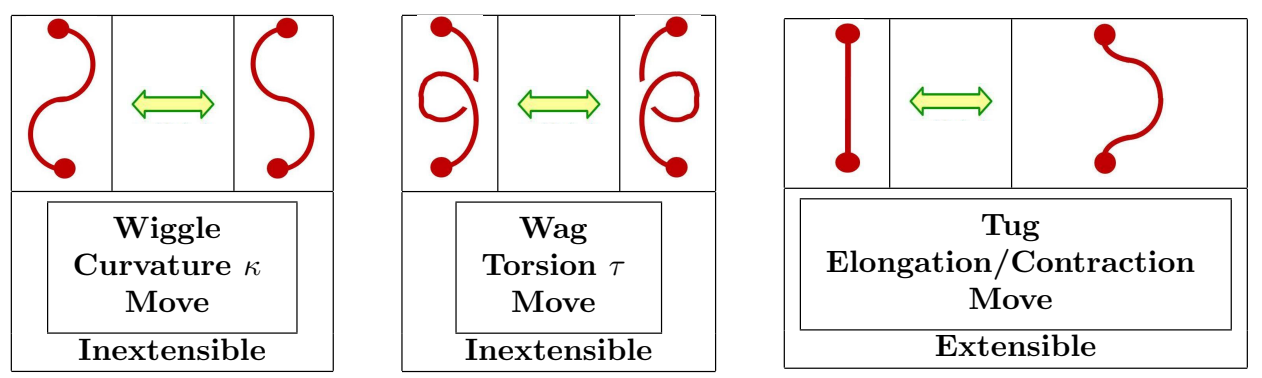

The easiest way to answer this question is to use a "scaffolding" for 3-space, i.e., the so called cubic honeycomb.

\section{Lattice knots}

For each non-negative integer $\ell$, let $\mathcal{L}_{\ell}$ denote the three dimensional lattice of points

$$
\mathcal{L}_{\ell}=\frac{1}{2^{\ell}} \mathbb{Z} \times \mathbb{Z} \times \mathbb{Z}=\left\{\left(\frac{m_{1}}{2^{\ell}}, \frac{m_{2}}{2^{\ell}}, \frac{m_{3}}{2^{\ell}}\right): m_{1}, m_{2}, m_{3} \in \mathbb{Z}\right\},
$$

lying in Euclidean 3 -space $\mathbb{R}^{3}$, where $\mathbb{Z}$ denotes the set of rational integers. This lattice determines a tiling of $\mathbb{R}^{3}$ by cubes of edge $2^{\ell}$, called the cubic honeycomb (a.k.a., the cubic tesselation) of order $\ell$.

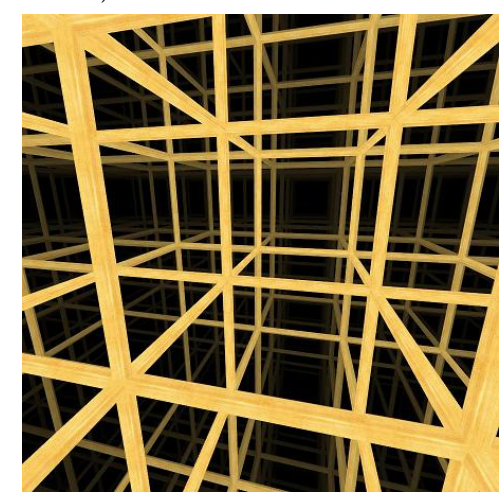

Cubic honeycomb of 3-space. [Figure taken from Wikipedia.]

We think of this honeycomb as a cell complex $\mathcal{C}_{\ell}$ for $\mathbb{R}^{3}$ consisting of $\mathbf{0}$-cells, 1cells, 2-cells, and 3-cells called respectively vertices, edges, faces, and cubes. 


\begin{tabular}{|llll|}
\hline Vertices & Edges & Faces & Cubes \\
& & & \\
\hline $\boldsymbol{a} \in L_{\ell}$ & $\boldsymbol{E}$ & $\boldsymbol{F}$ & \\
\hline
\end{tabular}

All cells of positive dimension are assumed to be open cells. Moreover, let $\mathcal{C}_{\ell}^{j}$ denote the $j$-skeleton of the cell complex $\mathcal{C}_{\ell}$ for $j=0,1,2,3$.

Definition 7.1. A lattice graph $G$ (of order $\ell$ ) is a finite subset of edges (together with their respective vertices) of the cubic honeycomb $\mathcal{C}_{\ell}$. A lattice knot $K$ (of order $\ell$ ) is a 2-valent lattice graph of order $\ell$. Moreover, let $\mathbb{G}^{(\ell)}$ and $\mathbb{K}^{(\ell)}$ respectively denote the set of all lattice graphs (of order $\ell$.) and the set of all lattice knots (of order $\ell$ ).

Reminder: Throughout this paper, the term "knot" will refer to both knots and links.

Two examples of lattice knots are illustrated in the figure below:

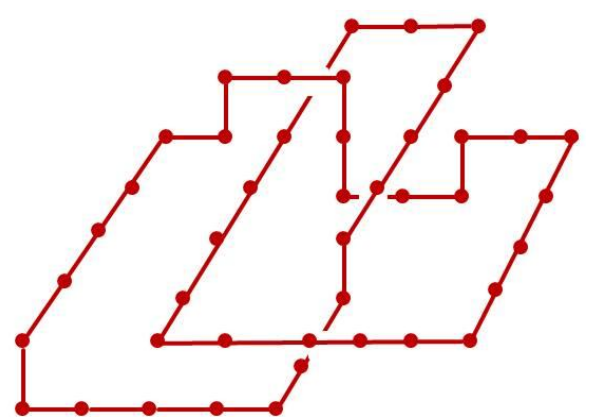

A lattice trefoil knot.

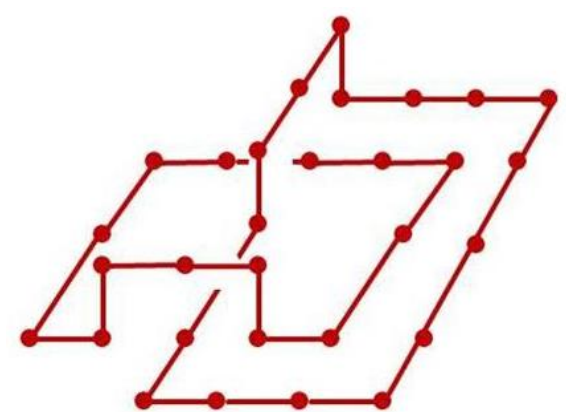

A lattice Hopf link.

\section{Basic terminology and conventions}

Before we can proceed further, we will need to create an infrastructure and nomenclature in which to work.

REMARK 8.1. The reader may find it convenient to quickly skim through this section, and later to refer back to it as needed. 
We define an orientation of Euclidean 3 -space $\mathbb{R}^{3}$ by selecting a right handed frame

$$
e=\left\{e_{1}=\left(\begin{array}{l}
1 \\
0 \\
0
\end{array}\right), e_{2}=\left(\begin{array}{l}
0 \\
0 \\
1
\end{array}\right), e_{3}=\left(\begin{array}{l}
0 \\
0 \\
1
\end{array}\right)\right\}
$$

at the origin, and by parallel transporting it to each vertex $a$ of the honeycomb $\mathcal{C}_{\ell}$. We will refer to this frame as the preferred frame.

Definition 8.2. A vertex $a$ of a cube $B$ is called the preferred vertex of cube $B$ if $B$ lies in the first octant of the preferred frame at $a$. Since $B$ is uniquely determined by its preferred vertex, we use the following notation:

$$
B=B^{(\ell)}(a) .
$$

The preferred edges and the preferred faces of the cube $B^{(\ell)}(a)$ are respectively the edges and faces of $B^{(\ell)}(a)$ that have $a$ as a vertex. We let

$$
E_{p}^{(\ell)}(a) \text { and } F_{p}^{(\ell)}(a)
$$

denote respectively the preferred edge parallel to the frame vector $e_{p}$ and the preferred face perpendicular to the frame vector $e_{p}$. The preferred edges of $F_{p}^{(\ell)}(a)$ are the edges of $F_{p}^{(\ell)}(a)$ that are preferred edges of the cube $B^{(\ell)}(a)$. Finally, $a$ is called the preferred vertex of the edge $E_{p}^{(\ell)}(a)$ and of the face $F_{p}^{(\ell)}(a)$

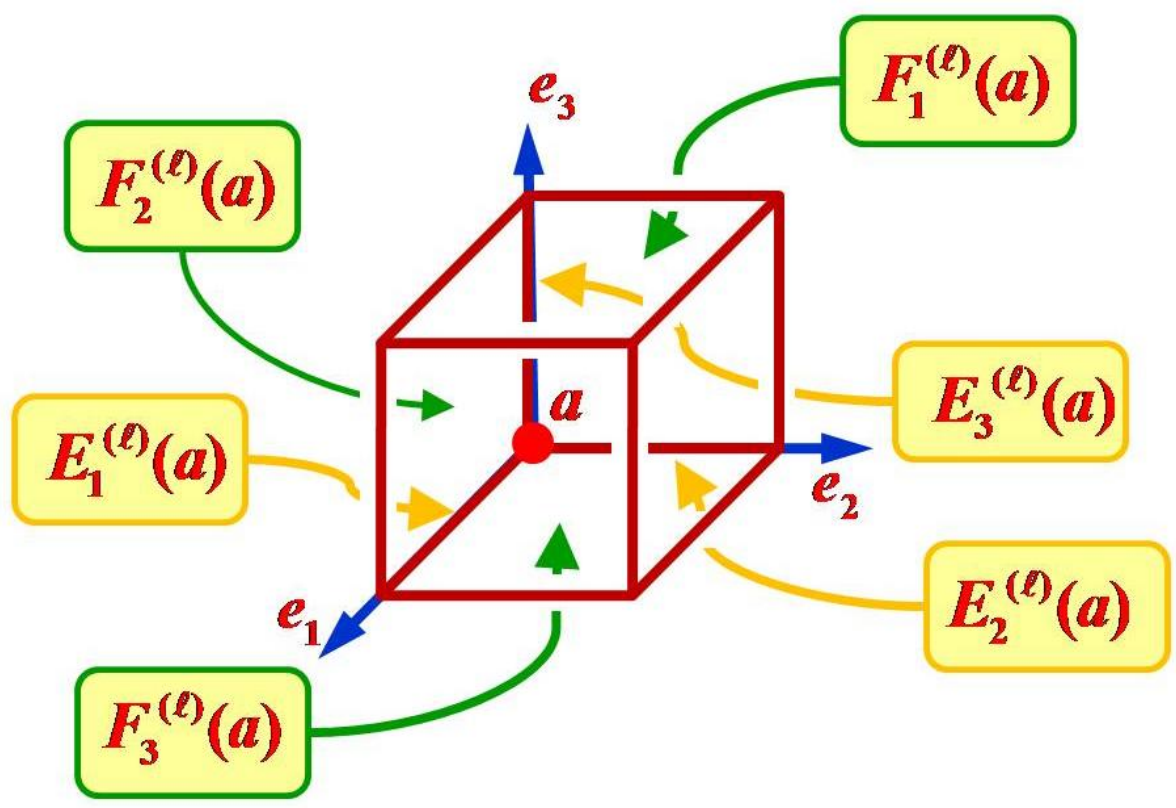

The preferred vertex, edges, and faces of the cube $B^{(\ell)}(a)$.

We will use the following drawing conventions: 
First drawing convention for cubes: Each cube $B^{(\ell)}(a)$, when drawn in isolation, is drawn with edges parallel to its preferred frame, and with its preferred vertex in the back bottom left hand corner.

First drawing convention for faces: Each face $F_{p}^{(\ell)}(a)$, when drawn in isolation, is always drawn with its preferred vertex a in the upper left hand corner, and with the frame vector $e_{p}(a)$ pointing out of the page. (Please refer to the figure below.)

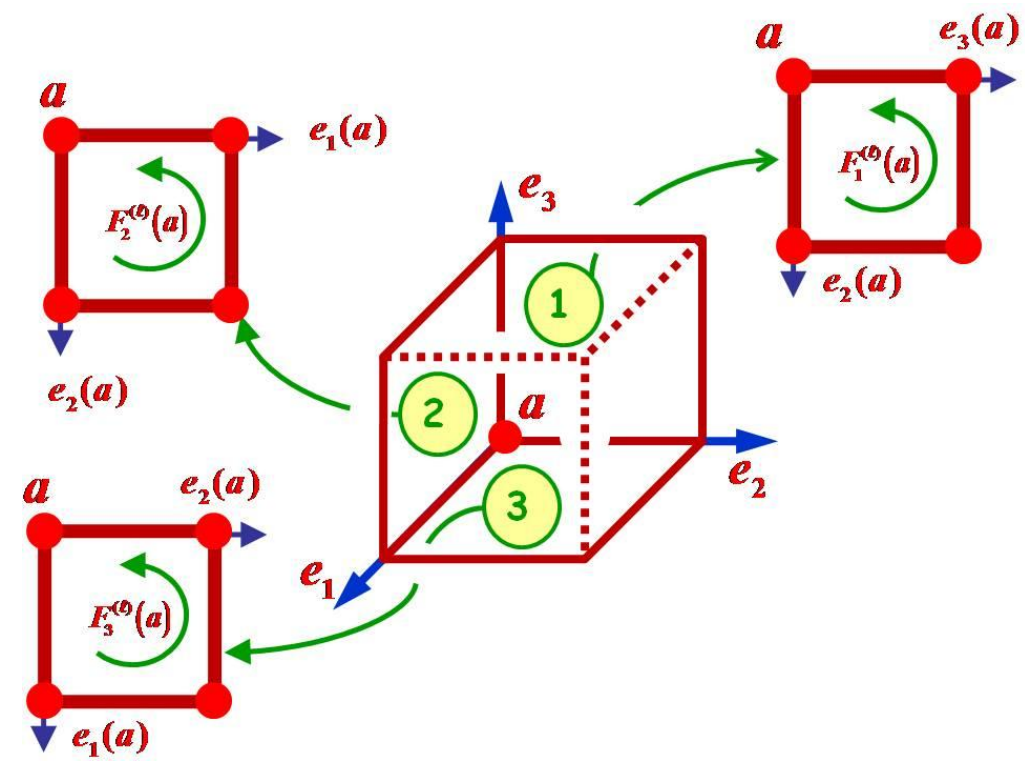

Drawing conventions for faces.

Second drawing convention for faces and cubes: We will also make use of the left and right permutations $\lfloor$ and $\rceil$ defined by

$$
\begin{aligned}
\lfloor:\{1,2,3\} & \longrightarrow\{1,2,3\} \\
1 & \longmapsto 2 \\
2 & \longmapsto 3 \\
3 & \longmapsto 1
\end{aligned}
$$

$$
\begin{aligned}
7:\{1,2,3\} & \longrightarrow\{1,2,3\} \\
1 & \longmapsto 3 \\
2 & \longmapsto 1 \\
3 & \longmapsto 2
\end{aligned}
$$

These permutations have been defined so that

$$
e_{p}=e_{\lfloor p} \times e_{p\rceil}, \quad e_{\lfloor p}=e_{p\rceil} \times e_{p}, \text { and } \quad e_{p\rceil}=e_{p} \times e_{\lfloor p}
$$

where ' $x$ ' denotes the right handed vector cross product. With the left and right permutations, the first drawing conventions for faces and cubes can now be more generally illustrated as shown below: 


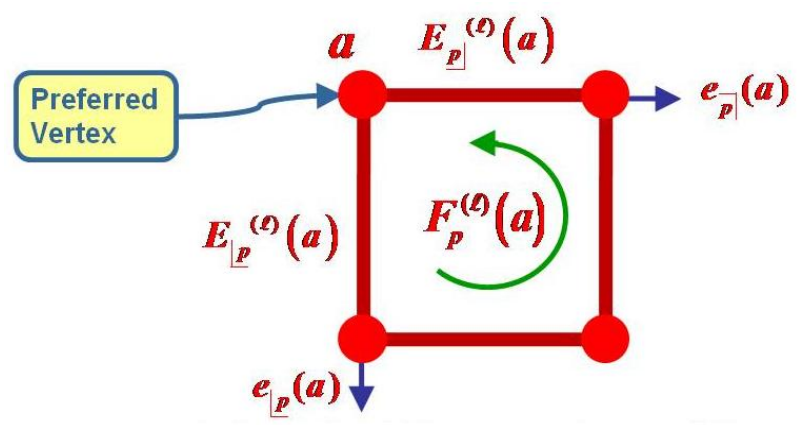

Face drawing conventions using the left and right permutations "L" and "ך". The frame vector $e_{p}(a)$ points out of the page

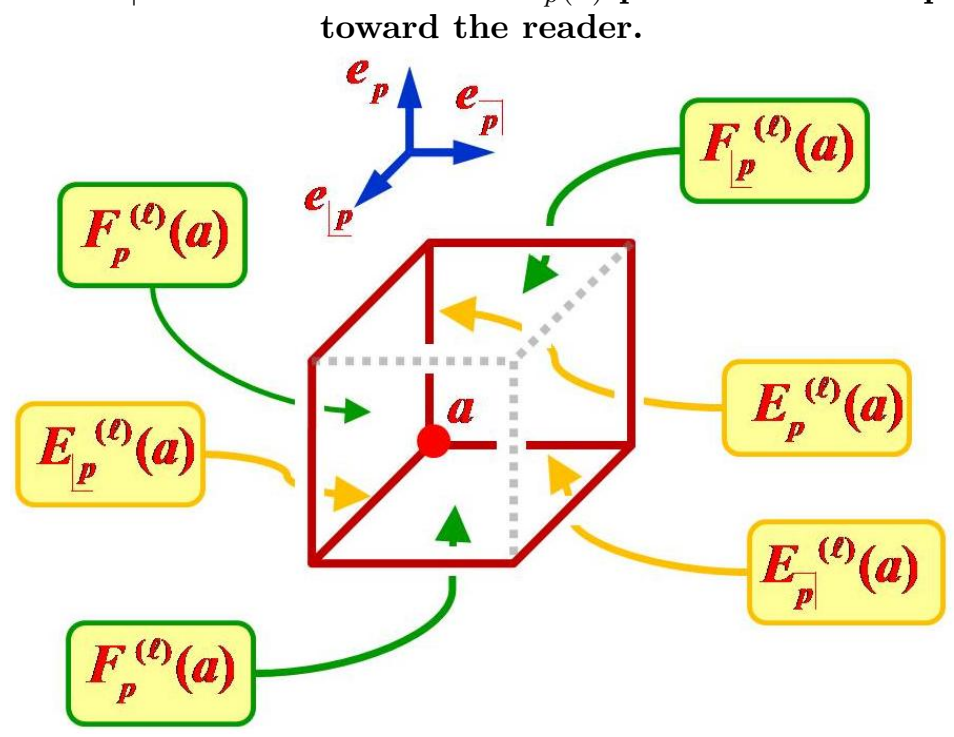

Cube drawing conventions using the left and right permutations $\lfloor$ and $\rceil$. $E$ :

We will use the following color coding scheme for the vertices $a$ and edges

\begin{tabular}{||c|c|c|}
\hline Solid Red & "Hollow" Gray & Solid Gray \\
\hline \hline & & \\
\hline Part of the lattice knot & Not part of the lattice knot & $\begin{array}{c}\text { Indeterminate, may } \\
\text { or may not be part } \\
\text { of the lattice knot. }\end{array}$ \\
\hline
\end{tabular}




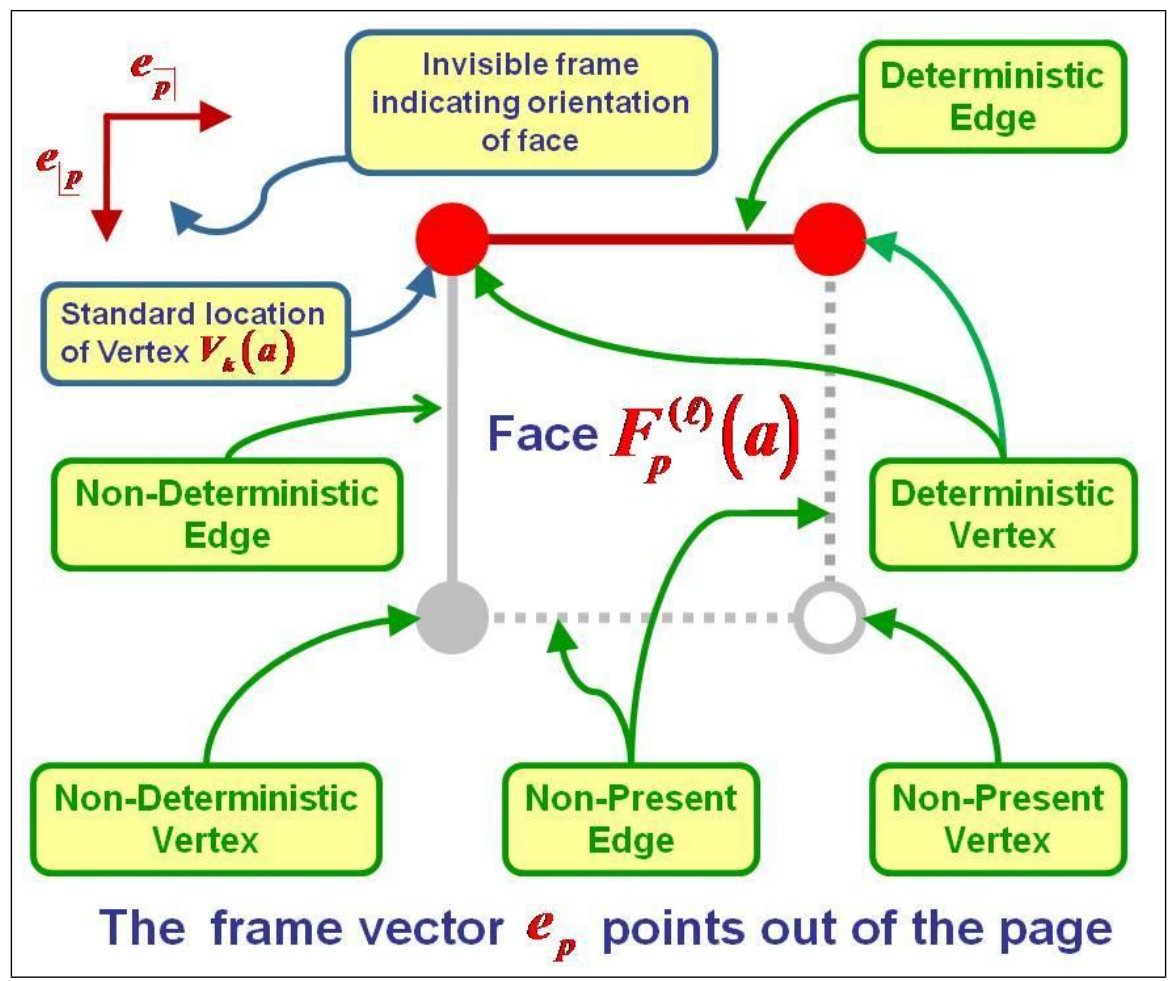

An illustration of the color coding scheme..

Finally, we will have need of the following definition:

Definition 8.3. For each integer $p=1,2,3$, we define the lattice translation map from the lattice $\mathcal{L}_{\ell}$ into itself as:

$$
\begin{aligned}
\top_{p}: \mathcal{L}_{\ell} & \longrightarrow \mathcal{L}_{\ell} \\
a & \longmapsto a+2^{-\ell} e_{p}
\end{aligned}
$$

where $e_{p}$ denotes the $p$-th unit length vector of the preferred frame. Moreover, we will often use the following more compact notation

$$
\top_{p} a=a^{: p} .
$$

For example, $a^{: 1^{2} \overline{2}^{3} 3}$ denotes

$$
a^{: 1^{2} \overline{2}^{3} 3}=\top_{1}^{2} \top_{2}^{-3} \top_{3} a=a+2 \cdot 2^{-\ell} e_{1}-3 \cdot 2^{-\ell} e_{2}+2^{-\ell} e_{3}
$$

REMARK 8.4. Throughout this paper, we have made an effort to devise a mathematical notation that is intuitive as well as non-cumbersome. We hope the reader will find that this is the case. 


\section{Lattice Knot Moves: Wiggle, Wag, and Tug}

Using the graphical conventions prescribed in the previous section, we now define, for each non-negative integer $\ell$, three lattice knot moves $L_{1}^{(\ell)}, L_{2}^{(\ell)}$, and $L_{3}^{(\ell)}$, called respectively tug, wiggle, and wag. Each lattice move is a bijection from the set of lattice knots $\mathbb{K}^{(\ell)}$ onto itself, i.e., a permutation of $\mathbb{K}^{(\ell)}$.

While reading this section, the reader may find it helpful to refer to notational summaries found in Appendix B.

9.1. Definition of the move tug. The first move, called a tug, and denoted by

$$
L_{1}^{(\ell)}(a, p, q)
$$

is defined for each of the four edges of each preferred face $F_{p}^{(\ell)}(a)$ of each cube $B^{(\ell)}(a)$ in the cell complex $\mathcal{C}_{\ell}$. As indicated in the figure given below, we index the four edges of a preferred face $F_{p}^{(\ell)}(a)$, beginning with the preferred edge $E_{\lfloor p}^{(\ell)}(a)$, with the integers $q=0,1,2,3$ (also respectively by the symbols $q=\mid]$, 드, 디 , $\square)$, using the counterclockwise orientation induced on the face $F_{p}^{(\ell)}(a)$ by the preferred frame $e$.

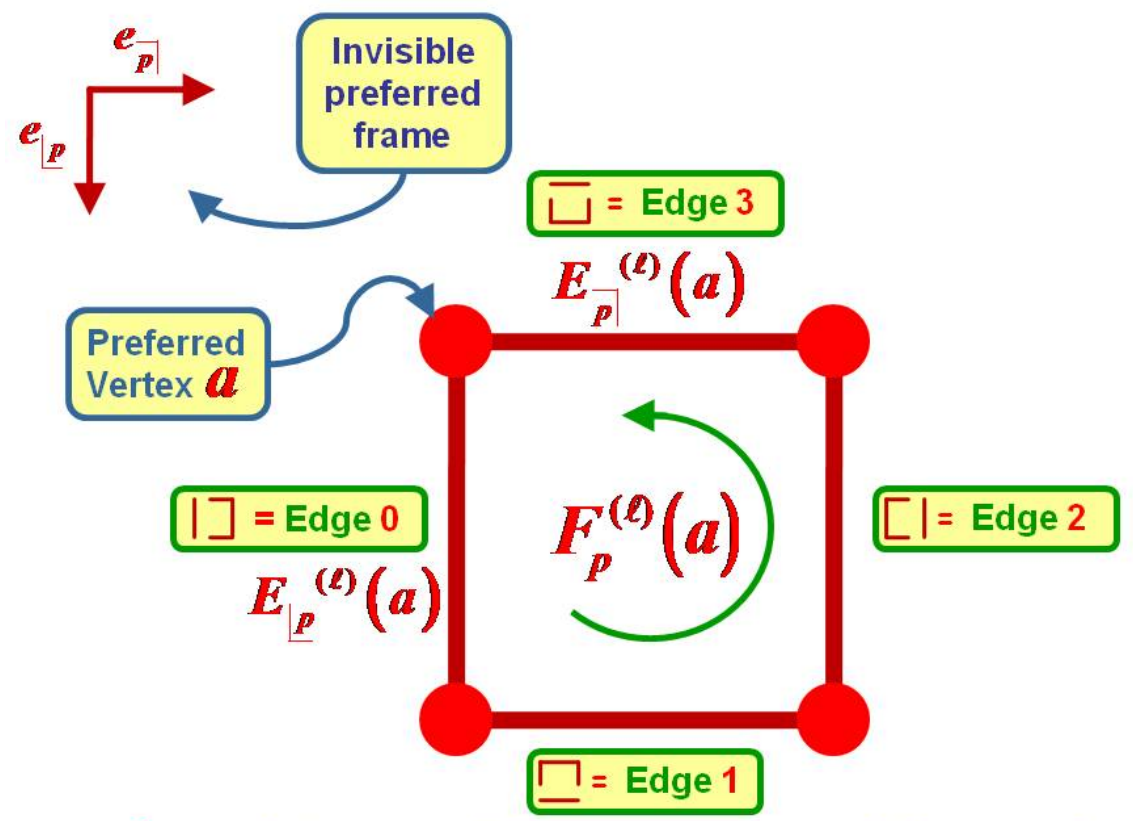

$\boldsymbol{e}_{p}$ points out of the page toward the reader

Edge ordering conventions for tug move $L_{1}^{(\ell)}(a, p, q)$, for edges $q=0,1,2,3$, which are also respectively denoted by $\mid]^{(\ell)}, \underline{\square}^{(\ell)},\left[l^{(\ell)}\right.$, and $\square^{(\ell)}$. 
The tug $L_{1}^{(\ell)}(a, p, q)$ associated with the edge $q=0$ (also denoted by $\left.q=\mid\right]$ ) of the preferred face $F_{p}^{(\ell)}(a)$ of the cube $B^{(\ell)}(a)$ will be denoted in anyone of the following three ways

$$
\left.L_{1}^{(\ell)}(a, p, \mid]\right)=L_{1}^{(\ell)}(a, p, 0)=\mid \beth^{(\ell)}(a, p) .
$$

The remaining tugs $L_{1}^{(\ell)}(a, p, q)$, for $q=1,2,3$ (also indicated respectively by $q=$ 므, $[I, \bar{\square})$, are denoted in like manner.

Definition 9.1. We define the tug, written $L_{1}^{(\ell)}(a, p, 0)$ (also denoted by $\mid \beth^{(\ell)}(a, p)$ and $\left.L_{1}^{(\ell)}(a, p, \mid \sqsupset)\right)$, associated with the 0 -th edge of the $p$-th preferred face $F_{p}^{(\ell)}(a)$ of the cube $B_{\ell}(a)$ as the move

$$
\begin{aligned}
& \mid]^{(\ell)}(a, p)(K)=
\end{aligned}
$$

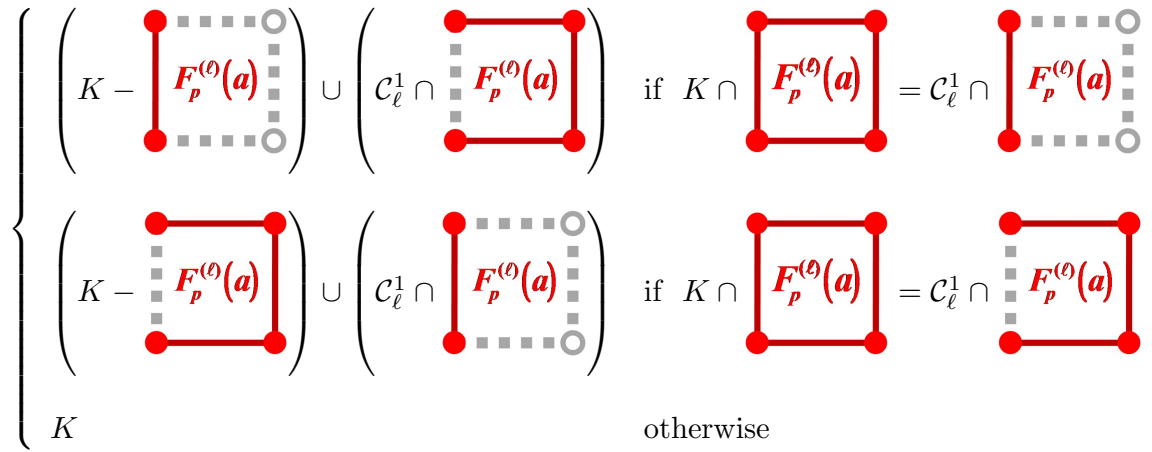

where $\boldsymbol{F}_{p}^{(\ell)}(\boldsymbol{a})=\boldsymbol{F}_{p}^{(\ell)}(\boldsymbol{a})$, and $\boldsymbol{F}_{p}^{(\ell)}(\boldsymbol{a})$ denote the 2-subcomplexes of the cell complex $\mathcal{C}_{\ell}$, as defined by the graphical conventions found in the previous section.

This tug, $\left.\left.L_{1}^{(\ell)}(a, p, 0)=L_{1}^{(\ell)}(a, p, \mid]\right)=\mid\right\rfloor^{(\ell)}(a, p)$ is more succinctly illustrated in the figure given below:
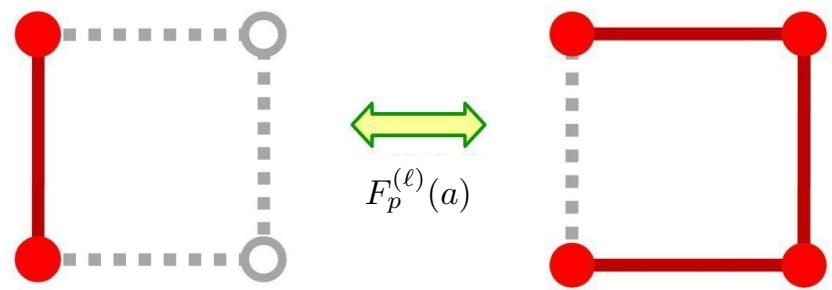

Lattice knot move $\left.\left.L_{1}^{(\ell)}(a, p, 0)=L_{1}^{(\ell)}(a, p, \mid]\right)=\mid\right\rfloor^{(\ell)}(a, p)$, called a tug. 
The remaining three tugs,

$$
\left\{\begin{array}{l}
L_{1}^{(\ell)}(a, p, 1)=L_{1}^{(\ell)}(a, p, \underline{\square})=\underline{\square}^{(\ell)}(a, p), \\
L_{1}^{(\ell)}(a, p, 2)=L_{1}^{(\ell)}(a, p, \underline{\square})=\sqsubset l^{(\ell)}(a, p), \text { and } \\
L_{1}^{(\ell)}(a, p, 3)=L_{1}^{(\ell)}(a, p, \bar{\square})=\square^{(\ell)}(a, p)
\end{array}\right.
$$

are defined in like manner, and illustrated in the three figures given below

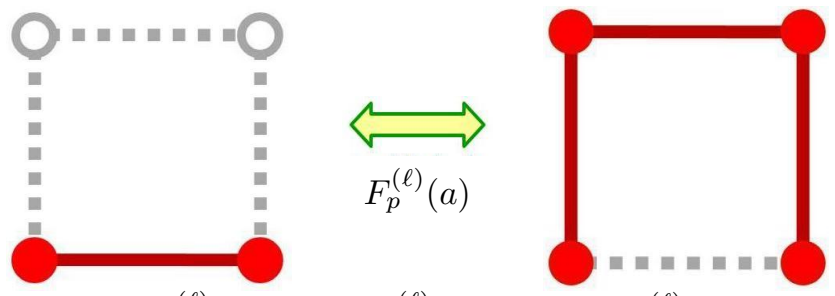

Lattice knot move $L_{1}^{(\ell)}(a, p, 1)=L_{1}^{(\ell)}(a, p, \underline{\square})=\underline{\square}^{(\ell)}(a, p)$, called a tug.

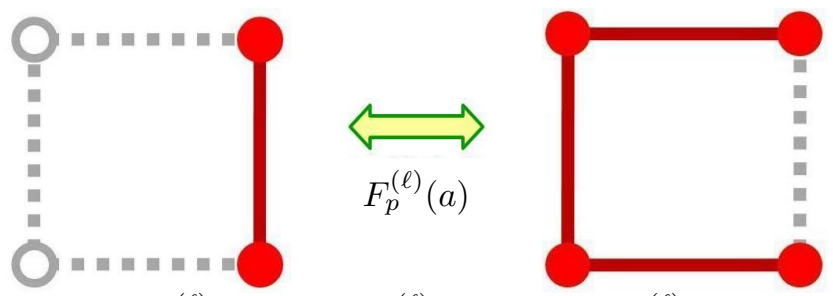

Lattice knot move $L_{1}^{(\ell)}(a, p, 2)=L_{1}^{(\ell)}\left(a, p,[\mid)=\left[l^{(\ell)}(a, p)\right.\right.$, called a tug.
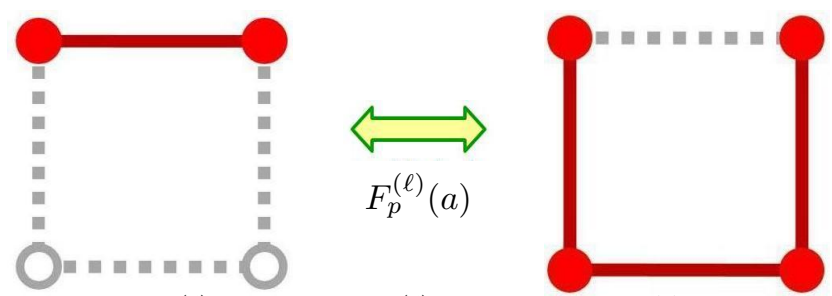

Lattice knot move $L_{1}^{(\ell)}(a, p, 3)=L_{1}^{(\ell)}(a, p, \square)=\square^{(\ell)}(a, p)$, called a tug.

Terminology: The designated edge of a tug move will be frequently called the tug's extendable edge.

REMARK 9.2. For each cube $B_{\ell}(a)$, there are 12 tug moves, i.e., 4 for each of the 3 preferred faces. 
9.2. Definition of the move wiggle. The second move, called a wiggle, and denoted by

$$
L_{2}^{(\ell)}(a, p, q)
$$

is defined for each of the two diagonals $q=$ '/' and $q=$ ' $\backslash$ ' of each preferred face $F_{p}^{(\ell)}(a)$ of each cube $B^{(\ell)}(a)$ in the cell complex $\mathcal{C}_{\ell}$.

For reasons that will soon become apparent, we will denote the diagonal $q=$ '/' by either the symbol

$$
q=\lceil
$$

or by either one of the integers

$$
q=0 \quad \text { or } \quad q=2 .
$$

Thus, the wiggle $L_{2}^{(\ell)}(a, p, q)$ with respect to diagonal $q=$ '/' of the preferred face $F_{p}^{(\ell)}(a)$ of the cube $B^{(\ell)}(a)$ will be denoted in anyone of the following three ways

$$
L_{2}^{(\ell)}(a, p,\ulcorner\lrcorner)=L_{2}^{(\ell)}(a, p, 0)=L_{2}^{(\ell)}(a, p, 2),
$$

or simply by

$$
\Gamma^{(\ell)}(a, p) \text {. }
$$

In like manner, we will denote the diagonal $q=$ '/' by either the symbol

$$
q=\llcorner
$$

or by either one of the integers

$$
q=1 \quad \text { or } \quad q=3 .
$$

Thus, the wiggle $L_{2}^{(\ell)}(a, p, q)$ with respect to diagonal $q=$ ' $\backslash$ ' of the preferred face $F_{p}^{(\ell)}(a)$ of the cube $B^{(\ell)}(a)$ will be denoted in anyone of the following three ways

$$
L_{2}^{(\ell)}\left(a, p,\llcorner)=L_{2}^{(\ell)}(a, p, 0)=L_{2}^{(\ell)}(a, p, 2),\right.
$$

or simply by

$$
\square^{(\ell)}(a, p)
$$

Definition 9.3. The wiggle associated with the diagonal \lceil\rfloor of the $p$-th preferred face $F_{p}^{(\ell)}(a)$ of the cube $B_{\ell}(a)$ on, written

$$
L_{2}^{(\ell)}(a, p,\ulcorner\rfloor)=L_{2}^{(\ell)}(a, p, 0)=L_{2}^{(\ell)}(a, p, 2)=\Gamma^{(\ell)}(a, p),
$$

is defined by 


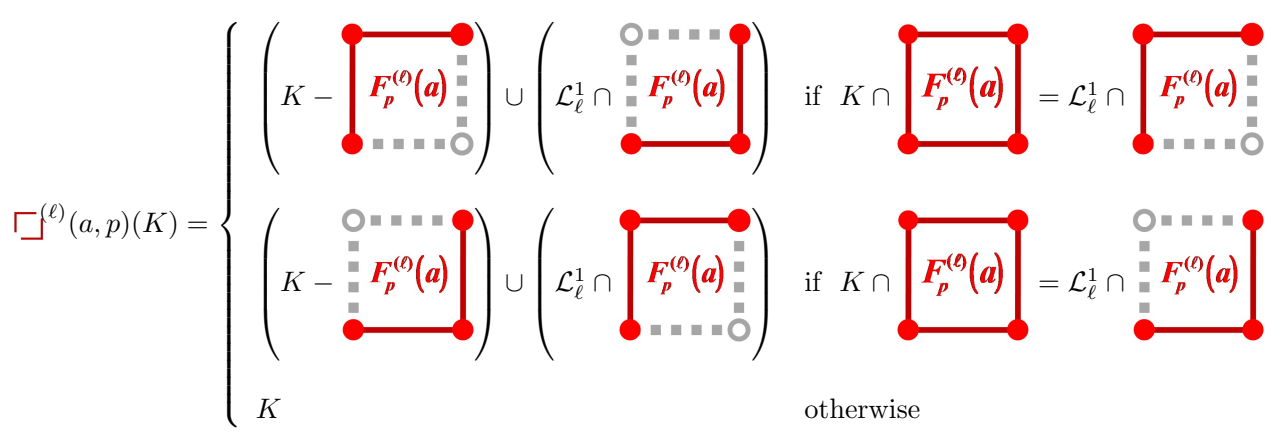

It is more succinctly illustrated in the figure given below:

\section{DEFINITION 9.4.}
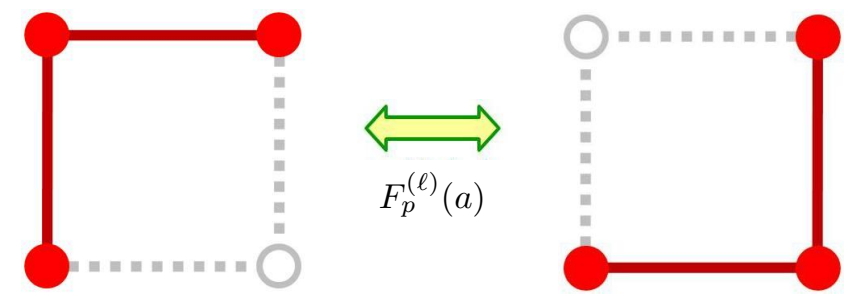

$$
L_{2}^{(\ell)}(a, p,\ulcorner\rfloor)=L_{2}^{(\ell)}(a, p, 0)=L_{2}^{(\ell)}(a, p, 2)=\left\ulcorner\square^{(\ell)}(a, p)\right.
$$

The lattice knot move, called a wiggle.

The remaining wiggle, $L_{2}^{(\ell)}(a, p, \square)$ is defined in like manner as

$$
\left\llcorner^{(\ell)}(a, p)(K)=\left\{\begin{array}{l}
\left(K-F_{p}^{(\ell)}(a)=\left(\mathcal{L}_{\ell}^{1} \cap F_{p}^{(\ell)}(a)\right]\right. \\
\left(\begin{array}{c}
K-F_{p}^{(\ell)}(a) \\
0
\end{array}\right) \cup\left(\mathcal{L}_{\ell}^{1} \cap F_{p}^{(\ell)}(a)\right. \\
K
\end{array}\right.\right.
$$

It is illustrated more succinctly in the figure given below 


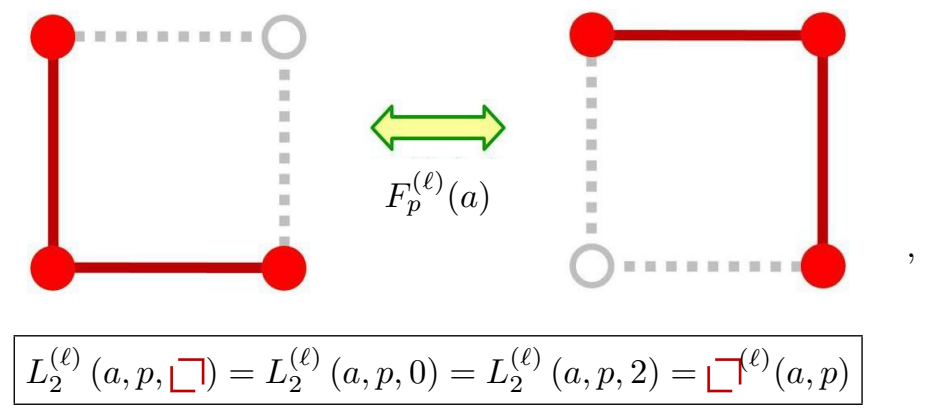

The lattice knot move, called a wiggle.

Remark 9.5. For each cube $B^{(\ell)}(a)$, there are 6 wiggle moves, 2 for each of the 3 preferred faces.

9.3. Definition of the move wag. The third move, called a wag, and denoted by

$$
L_{3}^{(\ell)}(a, p, q)
$$

is defined for each of the four perpendicular edges of a preferred face $F_{p}^{(\ell)}(a)$ of a cube $B^{(\ell)}(a)$ in the cell complex $\mathcal{C}_{\ell}$. As indicated in the figure given below, we index the four edges of the cube $B^{(\ell)}(a)$, which are perpendicular to a preferred face $F_{p}^{(\ell)}(a)$, beginning with the preferred edge $E_{p}^{(\ell)}(a)$ perpendicular to $F_{p}^{(\ell)}(a)$ at $a$, with the integers $0,1,2,3$ (or respectively with the symbols $\square, \square, \square, \square$ ), using the counterclockwise orientation induced on the face $F_{p}^{(\ell)}(a)$ by the preferred frame $e$. The chosen perpendicular edge will be called the hinge of the wag. 


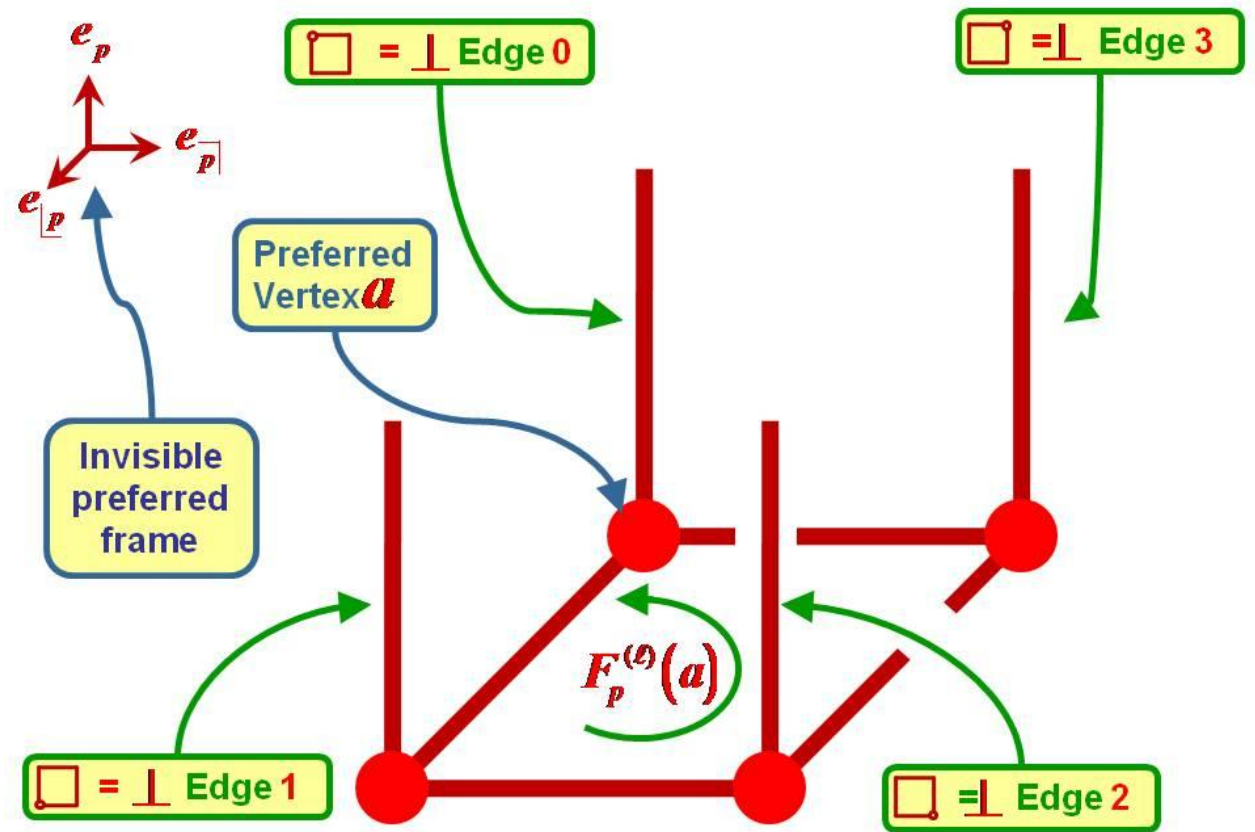

Edge ordering conventions for the $L_{3}^{(\ell)}(a, p, q)$ wag move, where $q=0,1,2,3$ (or respectively by $q=\square, \square, \square, \square$ ).

We display the figure below as a cryptic reminder for the reader of the notational conventions for the preferred edges and preferred faces of the cube $B^{(\ell)}(a)$ which are defined in a previous section of this paper:

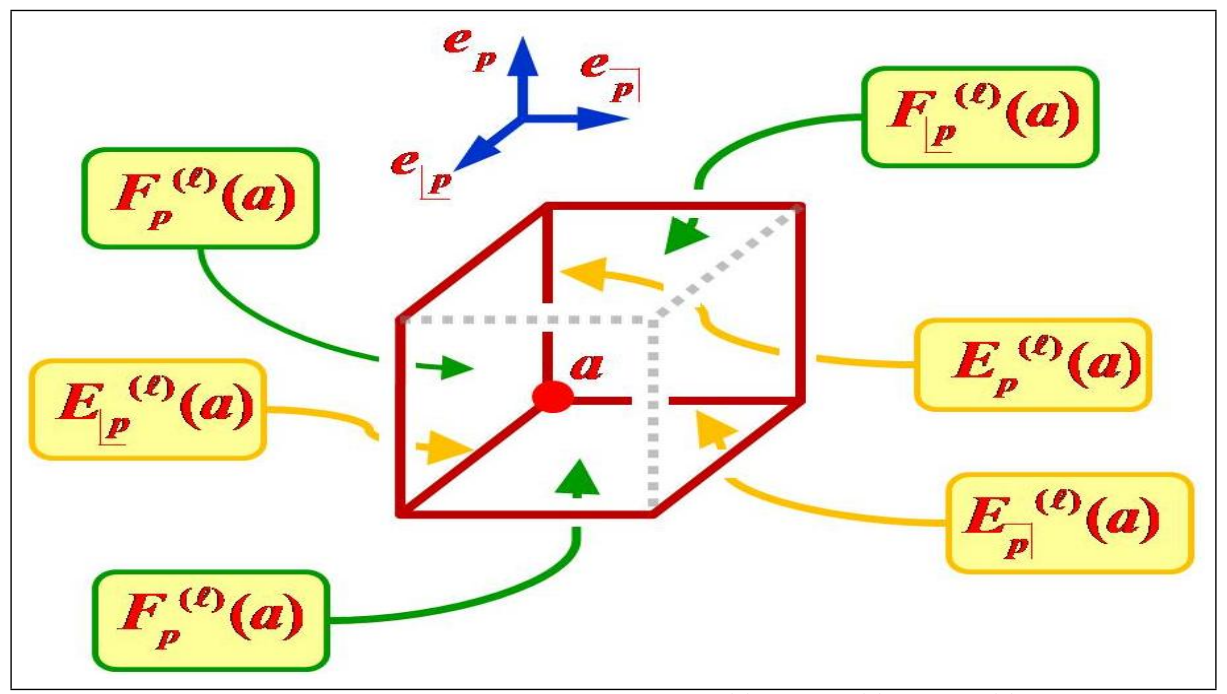

Preferred vertex $a$, preferred edges $E_{p}^{(\ell)}(a), E_{\lfloor p}^{(\ell)}(a), E_{p\rceil}^{(\ell)}(a)$, and preferred faces $F_{p}^{(\ell)}(a), F_{\lfloor p}^{(\ell)}(a), F_{p\rceil}^{(\ell)}(a)$ of cube $B^{(\ell)}(a)$. 
The wag $L_{3}^{(\ell)}(a, p, q)$ associated with the hinge $q=0$ (also denoted by $q=\emptyset$ ) of the preferred face $F_{p}^{(\ell)}(a)$ of the cube $B^{(\ell)}(a)$ will be denoted in any one of the following three ways

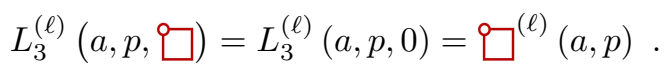

The remaining three tugs $L_{3}^{(\ell)}(a, p, q)$, for $q=1,2,3$ (also indicated respectively by $q=\square, \square, \square$ ), are denoted in like manner.

Definition 9.6. We define the wag, written $L_{3}^{(\ell)}(a, p, 0)[$ also denoted by $L_{3}^{(\ell)}(a, p, \bigcirc)$ and $\left.\square^{(\ell)}(a, p)\right]$, associated with the 0 -th perpendicular edge (called the 0 -th hinge) to the preferred face $F_{p}^{(\ell)}(a)$ of the cube $B^{(\ell)}(a)$ as the move

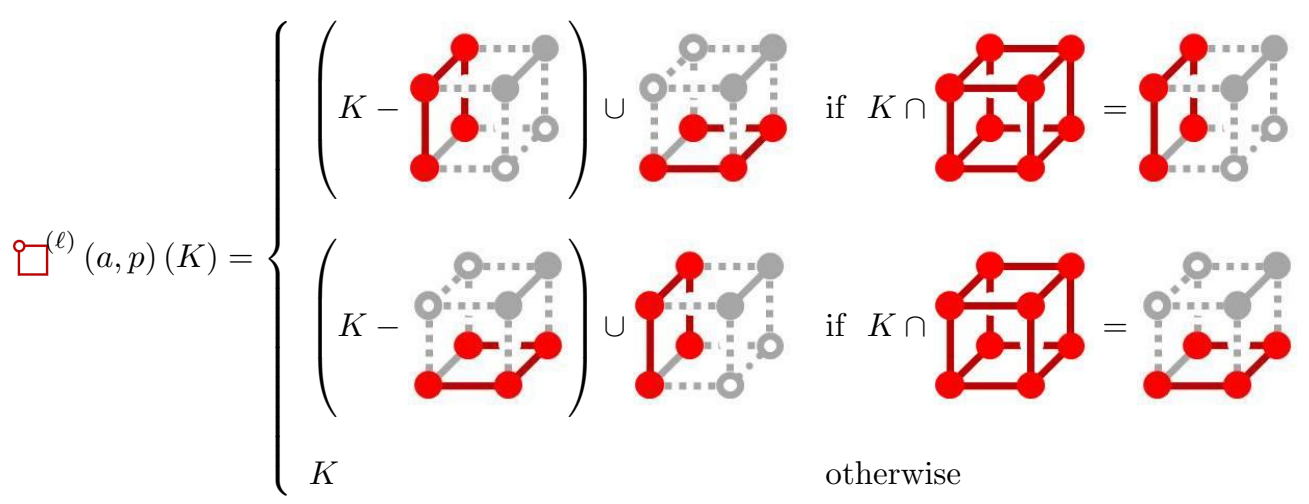

where, in each of the above graphics, the preferred face $F_{p}^{(\ell)}(a)$ is assumed to be the back face, and where
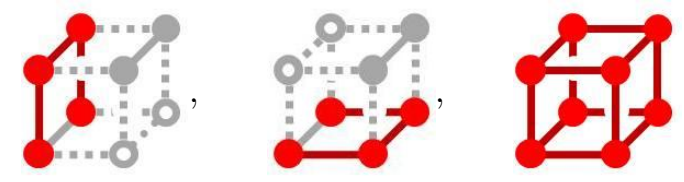

denote subcomplexes of the 1-skeleton of the boundary of the cube $B^{(\ell)}(a)$, as defined by the notational conventions found in the previous section, and where we have drawn the preferred face $F_{p}^{(\ell)}(a)$ as the back face in each of the above drawings.

This wag move $L_{3}^{(\ell)}(a, p, 0)=L_{3}^{(\ell)}(a, p, \square)=\square^{(\ell)}(a, p)$ is illustrated more succinctly in the figure given below 


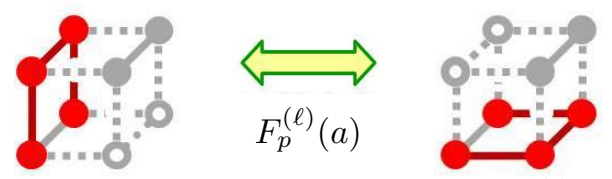

Lattice knot move $L_{3}^{(\ell)}(a, p, 0)=L_{3}^{(\ell)}(a, p, \emptyset)=\square^{(\ell)}(a, p)$, called a wag.

The other three tugs for face $F_{p}^{(\ell)}(a)$ are given below:
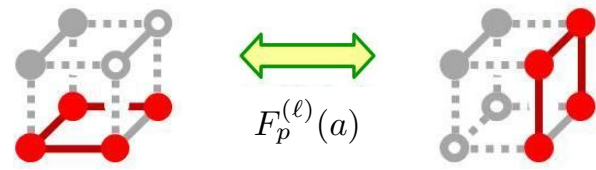

Lattice knot move $L_{3}^{(\ell)}(a, p, 1)=L_{3}^{(\ell)}(a, p, \square)=\square^{(\ell)}(a, p)$, called a wag.
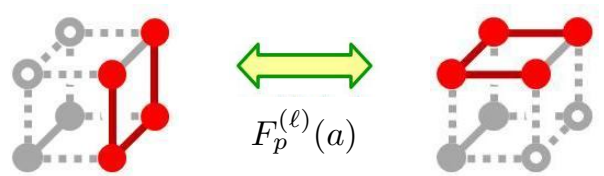

Lattice knot move $L_{3}^{(\ell)}(a, p, 2)=L_{3}^{(\ell)}(a, p, \square)=\square_{0}^{(\ell)}(a, p)$, called a wag.
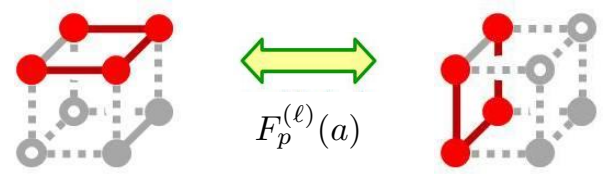

Lattice knot move $L_{3}^{(\ell)}(a, p, 3)=L_{3}^{(\ell)}(a, p, \square)=\square^{(\ell)}(a, p)$, called a wag.

Remark 9.7. For each cube $B^{(\ell)}(a)$, there are 12 wag moves, i.e., 4 for each of the 3 preferred faces.

9.4. Historical perspective. We should mention that the lattice moves tug and wiggle were first used toward the end of the ninetieth century by Dehn and Heegard. For more information, we refer the reader to $[\mathbf{8}]$ and $[\mathbf{4 0}]$.

\section{The ambient groups $\Lambda_{\ell}$ and $\widetilde{\Lambda}_{\ell}$}

The following proposition is an almost immediate consequence of the definitions of lattice knot moves given in the previous section.

Proposition 1. For each non-negative integer $\ell$, each lattice knot move $L_{m}^{(\ell)}(a, p, q)$ is a permutation of the set of all lattice knots $\mathbb{K}^{(\ell)}$ of order $\ell$. In fact, each is a permutation which is the product of disjoint transpositions. 
Proof. Let $L_{1}^{(\ell)}(a, p, q)$ be an arbitrary tug move, and let

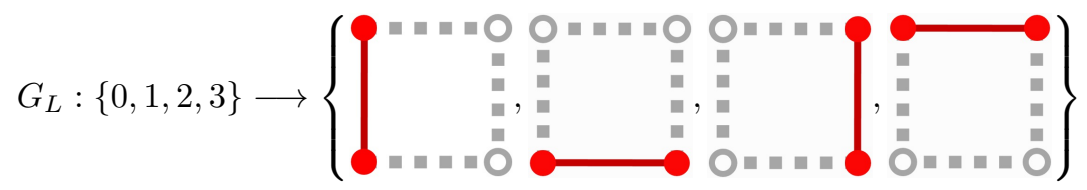

and

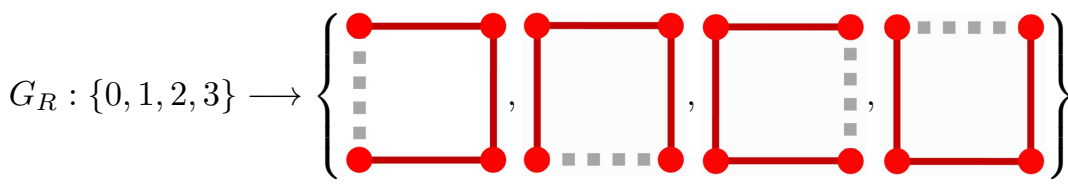

be the functions, from the set of integers $\{0,1,2,3\}$ into the above indicated set of symbols, defined by
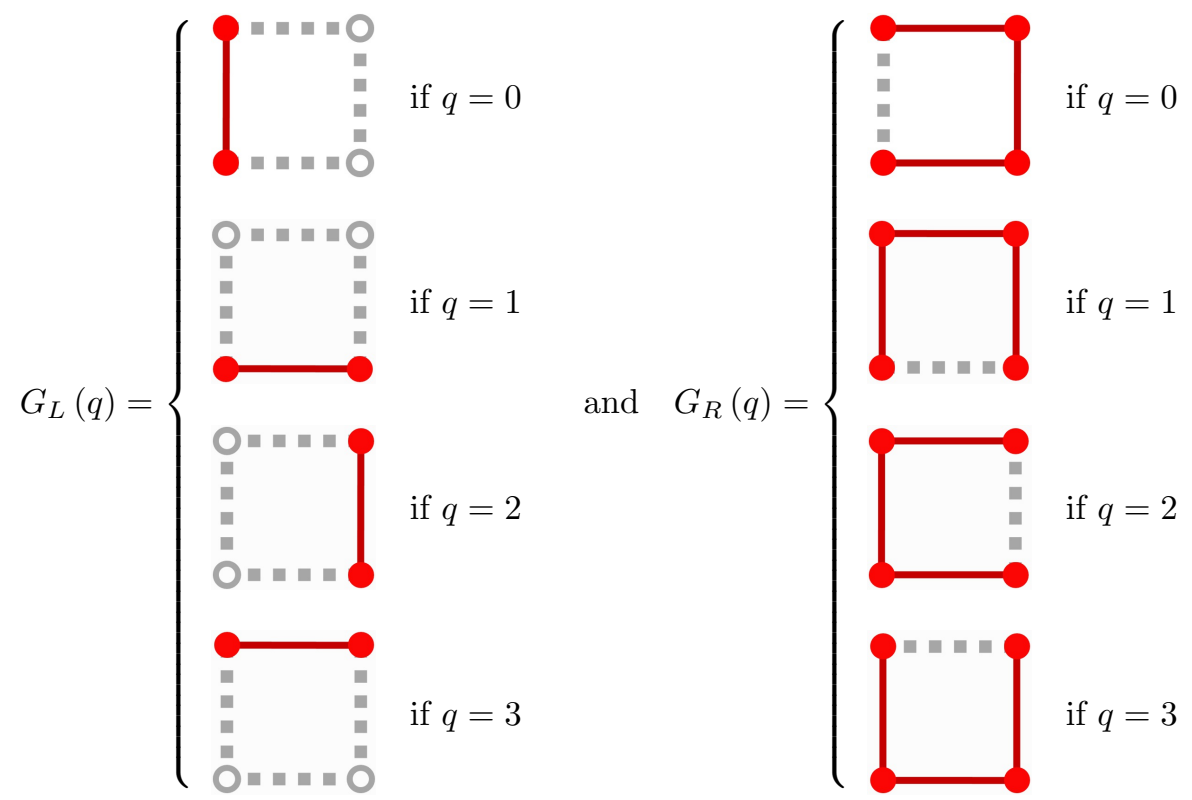

Then the definition of the tug move, which has been given in a previous section of this paper, can more succinctly be written as

$$
L_{1}^{(\ell)}(a, p, q)=G_{L}(q) \underset{F_{p}(a)}{\longleftrightarrow} G_{R}(q)
$$



by

Now let $\mathbb{K}_{L}^{(\ell)}(q)$ and $\mathbb{K}_{R}^{(\ell)}(q)$ be sets of order $\ell$ lattice knots respectively defined

$$
\begin{aligned}
& \mathbb{K}_{L}^{(\ell)}(q)=\left\{K \in \mathbb{K}^{(\ell)}: K \cap \boldsymbol{F}_{\boldsymbol{p}}^{(\ell)}(\boldsymbol{a})=G_{L}(q)\right\}, \text { and } \\
& \mathbb{K}_{R}^{(\ell)}(q)=\left\{K \in \mathbb{K}^{(\ell)}: K \cap \boldsymbol{F}_{\boldsymbol{p}}^{(\ell)}(\boldsymbol{a})=G_{R}(q)\right\} .
\end{aligned}
$$

Finally, let $\mathbb{K}_{R}^{(\ell)}(q)$ be the set of order $\ell$ lattice graphs defined by

$$
\mathbb{K}_{*}^{(\ell)}(q)=\mathbb{K}_{L}^{(\ell)}(q)-G_{L}(q)=\mathbb{K}_{R}^{(\ell)}(q)-G_{R}(q) .
$$

Then it immediately follows that $L_{1}^{(\ell)}(a, p, q)$ is the permutation

$$
L_{1}^{(\ell)}\left(a, p, q=\prod_{\alpha \in \mathbb{K}_{*}^{(\ell)}(q)}\left(\alpha \cup G_{L}(q), \alpha \cup G_{R}(q)\right),\right.
$$

where $\left(\alpha \cup G_{L}(q), \alpha \cup G_{R}(q)\right)$ is the transposition that interchanges the lattice knots $\alpha \cup G_{L}(q)$ and $\alpha \cup G_{R}(q)$.

For the remaining moves, i.e., for wiggles and wags, the proof is similar.

Since we have shown that tug, wiggle, and wag are permutations of the set of lattice knots, we can now give the following definition:

DEFINITION 10.1. For each non-negative integer $\ell$, we define the (lattice) ambient group

$\Lambda_{\ell}$

as the group of all permutations of the set $\mathbb{K}^{(\ell)}$ of lattice knots of order $\ell$ generated by the lattice knot moves tug, wiggle, and wag. Moreover, we define the inextensible (lattice) ambient group

$$
\widetilde{\Lambda}_{\ell}
$$

as the group of all permutations of the set $\mathbb{K}^{(\ell)}$ of lattice knots of order $\ell$ generated by the lattice knot moves wiggle and wag.

THEOREM 10.2. As abstract groups, all ambient groups $\Lambda_{\ell}$ are isomorphic, i.e., $\Lambda_{\ell} \simeq \Lambda_{\ell+1}$, for $\ell \geq 0$. More specifically,

$$
L_{m}^{(\ell)}(a, p, q) \longmapsto L_{m}^{(\ell)}\left(\frac{a}{2}, p, q\right)
$$

uniquely defines an isomorphism from $\Lambda_{\ell}$ onto $\Lambda_{\ell+1}$, for $\ell \geq 0$. The same is true for all inextensible ambient groups $\widetilde{\Lambda}_{\ell}$, i.e., $\widetilde{\Lambda}_{\ell} \simeq \widetilde{\Lambda}_{\ell+1}$, for $\ell \geq 0$. 
REMARK 10.3. Thus, as an abstract group, the ambient groups do not "see" the metric structure of Euclidean 3 -space $\mathbb{R}^{3}$. However, as we will later see, the metric structure can be found in the sequences of actions $\Lambda_{\ell} \times \mathbb{K}^{(\ell)} \longrightarrow \mathbb{K}^{(\ell)}$ and $\widetilde{\Lambda}_{\ell} \times \mathbb{K}^{(\ell)} \longrightarrow \mathbb{K}^{(\ell)}$.

At first, one might think that each wag can simply be written as a product of tugs. Surprisingly, the following theorem states that this is only conditionally true.

LEMmA 10.4. Let a be a vertex in the lattice $\mathcal{L}_{\ell}$. Then

$$
\square^{(\ell)}(a, 1)(K)=\left(\varpi^{(\ell)}(a, 2) \mid \Xi^{(\ell)}(a, 3) \varpi^{(\ell)}(a, 2)\right)(K)
$$

if and only if

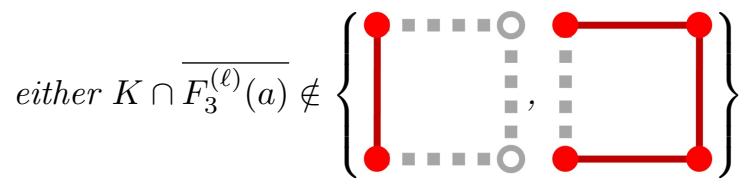

$$
\begin{aligned}
& \text { or } K \cap \overline{F_{2}^{(\ell)}(a)} \in\left\{\begin{array}{ll} 
& \\
0 &
\end{array}\right\}, \text { or both }
\end{aligned}
$$

and

if and only if

$$
\square^{(\ell)}(a, 1)(K)=\left(\left|\Xi^{(\ell)}(a, 3) \varpi^{(\ell)}(a, 2)\right| \Xi^{(\ell)}(a, 3)\right)(K)
$$

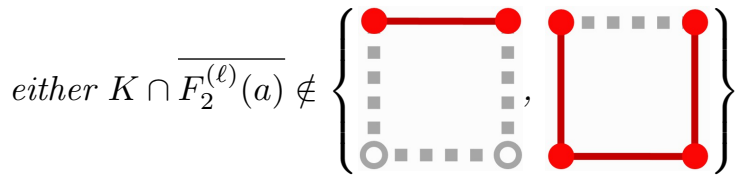

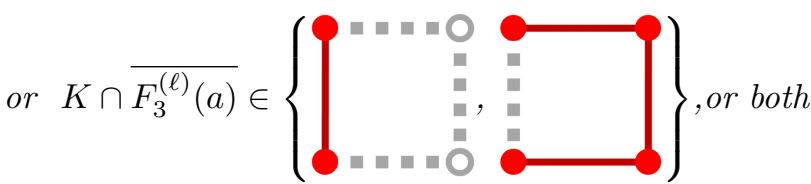

Similar statements can be made for the remaining 11 tugs.

COROLlary 1. For every wag $L_{3}^{(\ell)}(a, p, q)$ of order $\ell$ and for every lattice knot $K \in \mathbb{K}^{(\ell)}$, there is a finite sequence of tugs that transform $K$ into $L_{3}^{(\ell)}(a, p, q)(K)$. In this sense, every wag can be written as a finite product of tugs.

ExAMPLE 10.5. The following is an example of a lattice knot $K$ where

$$
\begin{aligned}
& L_{3}(a, 1, \square) K \neq L_{1}(a, 3, \mid \sqsupset) L_{1}(a, 2, \square) L_{1}(a, 3, \mid \sqsupset) K \\
& L_{3}(a, 1, \square) K=L_{1}(a, 2, \square) L_{1}(a, 3, \mid \sqsupset) L_{1}(a, 2,-\square) K
\end{aligned}
$$




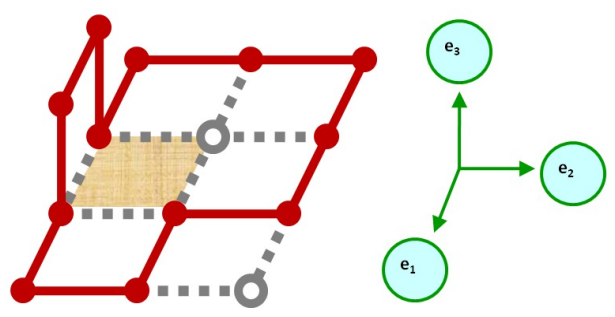

REMARK 10.6. The alert reader may also ask if there is an analogous lemma and corollary for wiggles. Unfortunately, this is not true because, unlike the more general wiggle move found in Section 5 of this paper, the lattice wiggle is a wiggle confined to a move only in a cubic lattice.

\section{Conditional auto-homeomorphism representations of $\Lambda_{\ell}$}

\section{Question. What is the intuitive meaning of the ambient group?}

We begin a search for an answer to this question by noting that the moves wiggle, wag, and tug are conditional symbolic moves, as are the Reidemeister moves. For example, the tug

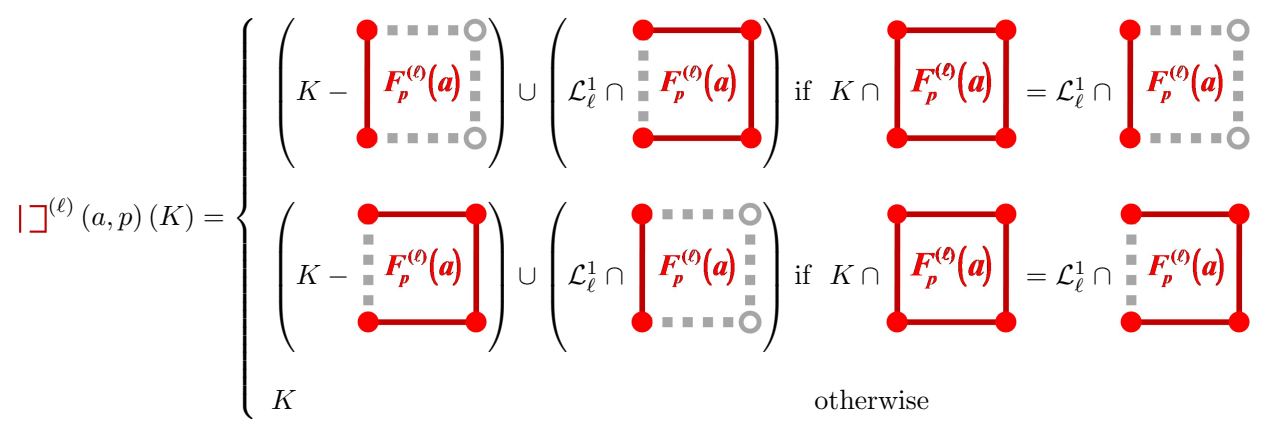

is a symbolic move based on a complete set of three mutually independent conditions.

Each such move is a symbolic representation of a conditional authentic move, i.e., a conditional orientation preserving (OP) auto-homeomorphism of $\mathbb{R}^{3}$. Moreover, each involved OP auto-homeomorphism

$$
h: \mathbb{R}^{3} \longrightarrow \mathbb{R}^{3}
$$

is local if there exists an closed 3-ball $D$ such that $h$ is the identity homeomorphism $i d$ on the complement of int $(D)$, i.e., such that

$$
\left.h\right|_{\mathbb{R}^{3}-i n t(D)}=i d: \mathbb{R}^{3}-\operatorname{int}(D) \longrightarrow \mathbb{R}^{3}-i n t(D),
$$

where $\operatorname{int}(D)$ denotes the interior of the closed 3-ball $D$. 
To complete the answer to our question, we will need the following definition.

DEFINITION 11.1. Let $L A H_{O P}\left(\mathbb{R}^{3}\right)$ be the group of orientation preserving (OP) local auto-homeomorphisms of 3-space $\mathbb{R}^{3}$. A conditional authentic move $\Phi$ for a family $\mathcal{F}$ of knots in 3 -space $\mathbb{R}^{3}$ is a map

$$
\begin{aligned}
\Phi: \mathcal{F} & \longrightarrow A H_{O P}\left(\mathbb{R}^{3}\right) \\
K & \longmapsto\left(\Phi_{K}: \mathbb{R}^{3} \longrightarrow \mathbb{R}^{3}\right)
\end{aligned}
$$

such that

$$
\Phi_{K}(K) \in \mathcal{F}
$$

for all $K \in \mathcal{F}$.

REMARK 11.2. It readily follows that the elements of the ambient groups $\Lambda_{\ell}$ and $\widetilde{\Lambda}_{\ell}$ are all conditional authentic moves for the family $\mathbb{K}^{(\ell)}$ of lattice knots of order $\ell$.

Definition 11.3. Let $L A H_{O P}\left(\mathbb{R}^{3}\right)^{\mathcal{F}}$ denote the space of all conditional OP local auto-homeomorphisms for a family of knots $\mathcal{F}$, together with the binary operation

$$
\begin{array}{ccc}
A H_{O P}\left(\mathbb{R}^{3}\right)^{\mathcal{F}} \times A H_{O P}\left(\mathbb{R}^{3}\right)^{\mathcal{K}} & & A H_{O P}\left(\mathbb{R}^{3}\right)^{\mathcal{F}} \\
\left(\Phi^{\prime}, \Phi\right) & \longmapsto & \Phi^{\prime} \cdot \Phi
\end{array}
$$

defined by

$$
\left(\Phi^{\prime} \cdot \Phi\right)=\Phi_{\Phi_{K}(K)}^{\prime} \circ \Phi_{K}
$$

where ' $\mathrm{O}$ ' denotes the composition of functions. This is readily seen to be a well defined binary operation on $L A H_{O P}\left(\mathbb{R}^{3}\right)^{\mathcal{F}}$.

REMARK 11.4. We should remind the reader that a knot $K$ is an imbedding $K: \bigsqcup_{j=1}^{s} S^{1} \longrightarrow \mathbb{R}^{3}$ of a disjoint union of finitely many circles into 3-space $\mathbb{R}^{3}$. Hence, $\Phi_{K}(K)$ is the imbedding $\Phi_{K} \circ K: \bigsqcup_{j=1}^{s} S^{1} \longrightarrow \mathbb{R}^{3}$, where 'o' denotes the composition of functions.

Proposition 2. The space $L A H_{O P}\left(\mathbb{R}^{3}\right)^{\mathcal{F}}$ together with the above binary operation '"' is a monoid.

Proof. Let $\Phi, \Phi^{\prime}, \Phi^{\prime \prime}$ be three arbitrary conditional authentic moves. Then $\left.\left(\Phi \cdot\left(\Phi^{\prime} \cdot \Phi^{\prime \prime}\right)\right)_{K}=\Phi_{\left(\Phi^{\prime} \cdot \Phi^{\prime \prime}\right)_{K}(K)} \circ\left(\Phi^{\prime} \cdot \Phi^{\prime \prime}\right)_{K}=\Phi_{\left(\Phi_{\Phi_{K}^{\prime \prime}(K)}^{\prime} \circ \Phi_{K}^{\prime \prime}\right)(K}\right) \circ \Phi_{\Phi_{K}^{\prime \prime}(K)}^{\prime} \circ \Phi_{K}^{\prime \prime}$

On the other hand,

$$
\left(\left(\Phi \cdot \Phi^{\prime}\right) \cdot \Phi^{\prime \prime}\right)_{K}=\left(\Phi \cdot \Phi^{\prime}\right)_{\Phi_{K}^{\prime \prime}(K)} \circ \Phi_{K}^{\prime \prime}=\Phi_{\left(\Phi_{\Phi_{K}^{\prime \prime}(K)}^{\prime} \circ \Phi_{K}^{\prime \prime}\right)(K)} \circ \Phi_{\Phi_{K}^{\prime \prime}(K)}^{\prime} \circ \Phi_{K}^{\prime \prime}
$$

Hence, ' $'$ ' is associative.

Let $i d: \mathbb{R}^{3} \longrightarrow \mathbb{R}^{3}$ be the identity homeomorphism. Then it easily follows that

$$
K \longmapsto i d: \mathbb{R}^{3} \longrightarrow \mathbb{R}^{3}
$$


is a conditional OP auto-homeomorphism which is an identity with respect to the binary operation ' $'$ '.

We will now construct a faithful representation

$$
\Gamma: \Lambda_{\ell} \longrightarrow L A H_{O P}\left(\mathbb{R}^{3}\right)^{\mathbb{K}^{(\ell)}}
$$

of the ambient group $\Lambda_{\ell}$ onto a subgroup of the monoid $\left(L A H_{O P}\left(\mathbb{R}^{3}\right)^{\mathbb{K}^{(\ell)}}, \cdot\right)$ by mapping each of the generators of $\Lambda_{\ell}$ onto a conditional OP local auto-homeomorpism of $\mathbb{R}^{3}$.

To define this representation, we need to construct for each of the generators $L_{m}^{(\ell)}(a, p, q)$ of $\Lambda_{\ell}$ a conditional OP local auto-homeomorphism $\Phi_{m, q}^{(\ell)}(a, p): \mathbb{R}^{3} \longrightarrow$ $\mathbb{R}^{3}$ such that

$L_{m}^{(\ell)}(a, p, q) K_{1}=L_{m}^{(\ell)}(a, p, q) K_{2} \quad$ if and only if $\quad \Phi_{m, q}^{(\ell)}(a, p) K_{1}=\Phi_{m, q}^{(\ell)}(a, p) K_{2}$

We now do so for the generators $L_{1}^{(\ell)}(a, 1,0), L_{2}^{(\ell)}(a, 1,0), L_{3}^{(\ell)}(a, 1,0)$. The construction is similar for the remaining generators.

11.1. Construction for tugs. For the tug $\left.L_{1}^{(\ell)}(a, 1,0)=\mid\right\rfloor^{(\ell)}(a, 1)$, we construct a conditional OP auto-homeomorphism $\Phi_{1,0}^{(\ell)}(a, 1): \mathbb{R}^{3} \longrightarrow \mathbb{R}^{3}$ as follows:

Let $\epsilon$ be a sufficiently small positive real number, let $c=\left(a+a^{: 23}\right) / 2$ be the center of the face $F_{1}^{(\ell)}(a)$, and let $D_{\epsilon}$ be the closed 3-cell bounded by the sphere

$$
\left|x-c-\epsilon e_{3}\right|^{2}=\left|a-c-\epsilon e_{3}\right|^{2}
$$

where $x=\left(x_{1}, x_{2}, x_{3}\right)$. Then

$$
\overline{F_{1}^{(\ell)}(a)} \subset D_{\epsilon} \quad \text { and } \quad \partial F_{1}^{(\ell)}(a) \cap \partial D_{\epsilon}=\left\{a, a^{: 2}\right\}
$$

where $\bar{C}$ and $\partial C$ respectively denote the closure and the boundary of a cell $C$. Now let $h: D_{\epsilon} \longrightarrow D_{\epsilon}$ be an OP auto-homeomorpism of the 3-cell $D_{\epsilon}$ such that

$$
h\left(\overline{E_{2}^{(\ell)}(a)}\right)=\overline{E_{3}^{(\ell)}(a)} \cup \overline{E_{2}^{(\ell)}\left(a^{: 3}\right)} \cup \overline{E_{3}^{(\ell)}\left(a^{: 2}\right)} \quad \text { and }\left.\quad h\right|_{\partial D_{\epsilon}}=\left.i d\right|_{\partial D_{\epsilon}}
$$

where $i d$ is the identity auto-homeomorphism of $\mathbb{R}^{3}$. 


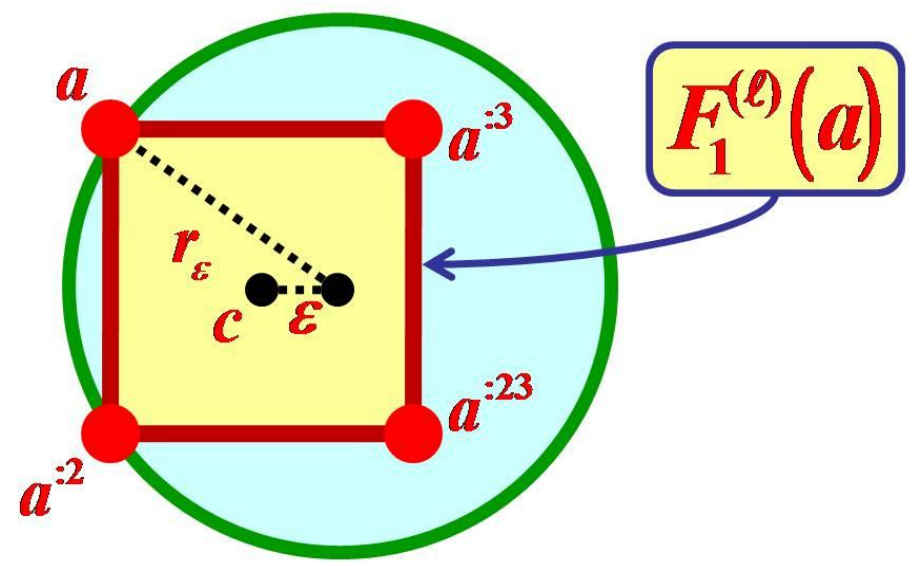

Cross Section of the 3-cell $D_{\varepsilon}$, where $r_{\varepsilon}=\left|a-c-\epsilon e_{3}\right|$.

Then we define $\Phi_{1,0}^{(\ell)}(a, 1)$ as

$\Phi_{1,0}^{(\ell)}(a, 1)_{K}= \begin{cases}h & \text { if } K \cap F_{1}^{(\ell)}(a)=\bar{E}_{2}^{(\ell)}(a) \text { and } x \in D_{\epsilon} \\ h^{-1} & \text { if } K \cap F_{1}^{(\ell)}(a)=\overline{E_{3}^{(\ell)}(a)} \cup \overline{E_{2}^{(\ell)}\left(a^{: 3}\right)} \cup \overline{E_{3}^{(\ell)}\left(a^{: 2}\right)} \text { and } x \in D_{\epsilon} \\ i d & \text { otherwise }\end{cases}$

11.2. Construction for wiggles. For the wiggle $L_{2}^{(\ell)}(a, 1,0)=\Gamma^{(\ell)}(a, 1)$, we construct a conditional OP auto-homeomorphism $\Phi_{2,0}^{(\ell)}(a, 1): \mathbb{R}^{3} \longrightarrow \mathbb{R}^{3}$ as follows:

Let $\epsilon$ be a sufficiently small positive real number, and let $D_{\epsilon}$ be the closed 3-cell bounded by the ellipsoid with axes

$\operatorname{Seg}\left(a^{: 2}, a^{: 3}\right), \quad \operatorname{Seg}\left(c-\sqrt{2} \cdot 2^{-\ell-1} e_{1}, c+\sqrt{2} \cdot 2^{-\ell-1} e_{1}\right), \operatorname{Seg}\left(a-\epsilon\left(e_{2}+e_{3}\right), a^{: 23}+\epsilon\left(e_{2}+e_{3}\right)\right)$

where $c=\left(a^{: 2}+a^{: 3}\right) / 2$ is the center of the face $F_{1}^{(\ell)}(a)$, and where $\operatorname{Seg}\left(b, b^{\prime}\right)$ denotes the line segment connecting points $b$ and $b^{\prime}$. Then

$$
\overline{F_{1}^{(\ell)}(a)} \subset D_{\epsilon} \quad \text { and } \quad \partial F_{1}^{(\ell)}(a) \cap \partial D_{\epsilon}=\left\{a^{: 2}, a^{: 3}\right\},
$$

where $\bar{C}$ and $\partial C$ respectively denote the closure and the boundary of a cell $C$. 


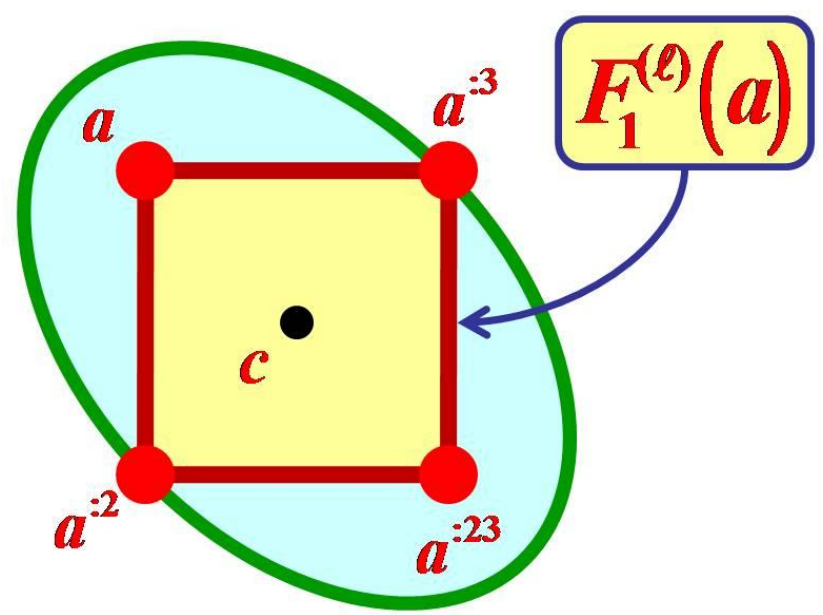

Cross section of 3-cell $D_{\varepsilon}$.

Now let $h: D_{\epsilon} \longrightarrow D_{\epsilon}$ be an OP auto-homeomorpism of the 3-cell $D_{\epsilon}$ such that

$$
h\left(\overline{E_{2}^{(\ell)}(a)} \cup \overline{E_{3}^{(\ell)}(a)}\right)=\overline{E_{2}^{(\ell)}\left(a^{: 3}\right)} \cup \overline{E_{3}^{(\ell)}\left(a^{: 2}\right)} \quad \text { and }\left.\quad h\right|_{\partial D_{\epsilon}}=\left.i d\right|_{\partial D_{\epsilon}},
$$

where $i d$ is the identity auto-homeomorphism of $\mathbb{R}^{3}$.

Then we define $\Phi_{2,0}^{(\ell)}(a, 1)$ as

$$
\Phi_{2,0}^{(\ell)}(a, 1)_{K}= \begin{cases}h & \text { if } K \cap F_{1}^{(\ell)}(a)=\overline{E_{2}^{(\ell)}(a)} \cup \overline{E_{3}^{(\ell)}(a)} \text { and } x \in D_{\epsilon} \\ h^{-1} & \text { if } K \cap F_{1}^{(\ell)}(a)=\overline{E_{2}^{(\ell)}\left(a^{: 3}\right)} \cup \overline{E_{3}^{(\ell)}\left(a^{: 2}\right)} \text { and } x \in D_{\epsilon} \\ i d & \text { otherwise }\end{cases}
$$

11.3. Construction for wags. For the wag $L_{3}^{(\ell)}(a, 1,0)=\bigcup^{(\ell)}(a, 1)$, we construct a conditional OP auto-homeomorphism $\Phi_{3,0}^{(\ell)}(a, 1): \mathbb{R}^{3} \longrightarrow \mathbb{R}^{3}$ as follows:

We wish to construct a closed 3-cell $D_{\epsilon}$ such that

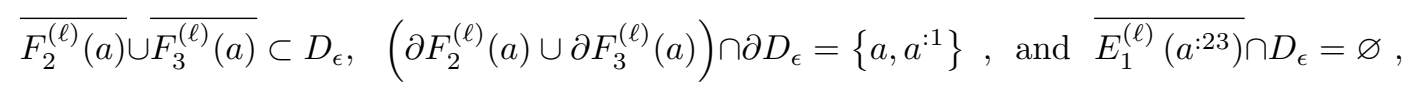

where $\bar{C}$ and $\partial C$ respectively denote the closure and the boundary of a cell $C$. We do so as follows:

Let $\epsilon$ be a sufficiently small positive real number, let $c$ be the center of the cube $B^{(\ell)}(a)$, and let $D_{\epsilon}^{\prime}$ be the closed 3 -cell bounded by the sphere with center

and of radius

$$
c+\epsilon\left(\frac{e_{2}+e_{3}}{\sqrt{2}}\right)
$$

$$
r=\left|a-c-\epsilon\left(\frac{e_{2}+e_{3}}{\sqrt{2}}\right)\right|
$$


Let $M$ be the plane passing through the point

$$
c+\frac{e_{2}+e_{3}}{3 \sqrt{2}}
$$

with normal $\left(e_{2}+e_{3}\right) / \sqrt{2}$. Then $\mathbb{R}^{3}-M$ consists of two disjoint open components $\mathbb{R}_{+}^{3}$ and $\mathbb{R}_{-}^{3}$, where $\mathbb{R}_{+}^{3}$ is that component into which the normal $\left(e_{2}+e_{3}\right) / \sqrt{2}$ points. Let $D_{\epsilon}$ be the closed 3-cell

$$
D_{\epsilon}=D_{\epsilon}^{\prime}-\mathbb{R}_{-}^{3} .
$$

Now let $h: D_{\epsilon} \longrightarrow D_{\epsilon}$ be an OP auto-homeomorpism of the 3-cell $D_{\epsilon}$ such that

$h\left(\overline{E_{3}^{(\ell)}(a)} \cup \overline{E_{1}^{(\ell)}\left(a^{: 3}\right)} \cup \overline{E_{3}^{(\ell)}\left(a^{: 1}\right)}\right)=\overline{E_{2}^{(\ell)}(a)} \cup \overline{E_{1}^{(\ell)}\left(a^{: 2}\right)} \cup \overline{E_{2}^{(\ell)}\left(a^{: 1}\right)} \quad$ and $\left.\quad h\right|_{\partial D_{\epsilon}}=\left.i d\right|_{\partial D_{\epsilon}}$,

where $i d$ is the identity auto-homeomorphism of $\mathbb{R}^{3}$.

We can now define $\Phi_{3,0}^{(\ell)}(a, 1)$ as

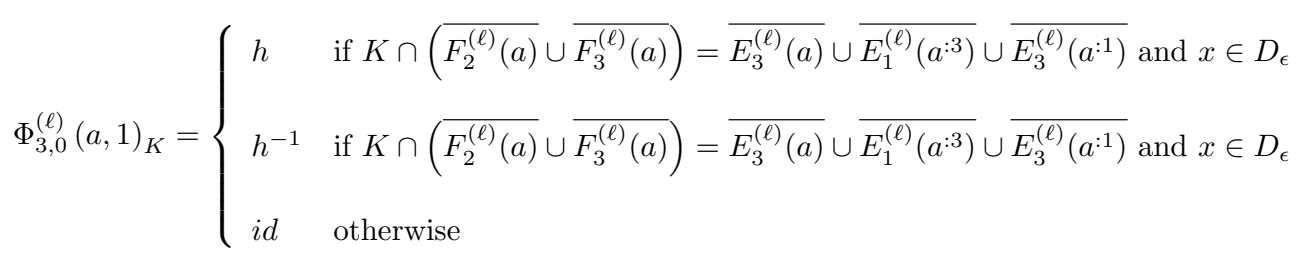

We leave it as an exercise for the reader to verify that the above constructed elements $\Phi_{m, q}^{(\ell)}(a, p)$ of $L A H_{O P}\left(\mathbb{R}^{3}\right)^{\mathbb{K}^{(\ell)}}$ are invertible in the monoid $L A H_{O P}\left(\mathbb{R}^{3}\right)^{\mathbb{K}^{(\ell)}}$. Hence we have:

Proposition 3. A faithful representation

$$
\Gamma: \Lambda_{\ell} \longrightarrow L A H_{O P}\left(\mathbb{R}^{3}\right)^{\mathbb{K}^{(\ell)}}
$$

of the ambient group $\Lambda_{\ell}$ onto a subgroup of the monoid $\left(L A H_{O P}\left(\mathbb{R}^{3}\right)^{\mathbb{K}^{(\ell)}}, \cdot\right)$ is uniquely determined by

$$
\Gamma: L_{m}^{(\ell)}(a, p, q) \longmapsto \Phi_{m, q}^{(\ell)}(a, p)
$$

REMARK 11.5. Please note that the above construction of the faithful representation $\Gamma$ is far from unique. 


\section{The refinement injection and the conjectured refinement morphism}

Definition 12.1. We define the refinement injection $Я: \mathbb{K}^{(\ell)} \longrightarrow \mathbb{K}^{(\ell+1)}$ from the set of lattice knots $\mathbb{K}^{(\ell)}$ of order $\ell$ to the set $\mathbb{K}^{(\ell+1)}$ of lattice knots of order $\ell+1$ as

$$
\begin{aligned}
\text { Я : } \mathbb{K}^{(\ell)} & \longrightarrow \mathbb{K}^{(\ell+1)} \\
K & \longmapsto \bigcup_{a \in \mathcal{L}_{\ell}} \bigcup_{p=1}^{3} \bigcup_{E_{p}^{(\ell)}(a) \in K}\left\{\overline{E_{p}^{(\ell+1)}}(a), \overline{E_{p}^{(\ell+1)}}\left(a^{: p}\right)\right\}
\end{aligned}
$$

where $\overline{E_{p}^{(\ell+1)}}$ denotes the closure of the open edge $E_{p}^{(\ell+1)}$.
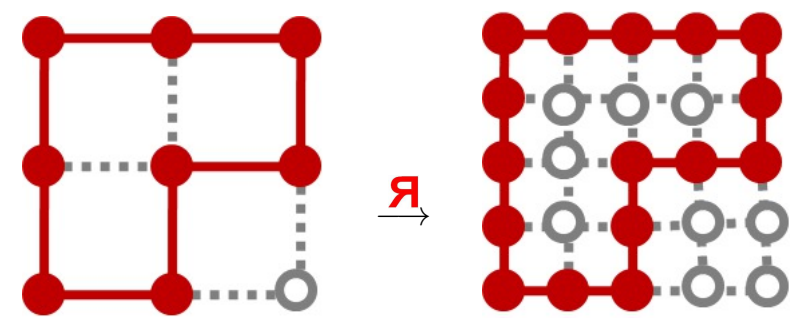

An example of the refinement injection 9 .

We would also like to construct a refinement map

$$
\text { я : } \Lambda_{\ell} \longrightarrow \Lambda_{\ell+1}
$$

that

(a) Is a group monomorphism, and

(b) Preserves the actions of the ambient groups $\Lambda_{\ell}$ and $\Lambda_{\ell+1}$ on the sets $\mathbb{K}^{(\ell)}$ and $\mathbb{K}^{(\ell+1)}$, respectively. In other words, we would like the following diagram to be commutative:

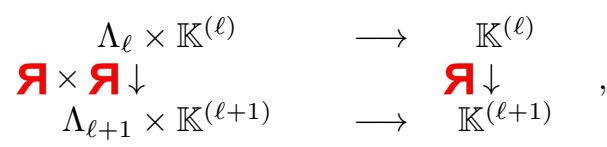

Appendix $\mathrm{C}$ gives a suggested construction for such a refinement map Я : $\Lambda_{\ell} \longrightarrow \Lambda_{\ell+1}$. It also gives a rationale for the following conjectures:

Conjecture 1A. The Appendix $C$ construction produces a well-defined map 9 : $\Lambda_{\ell} \longrightarrow \Lambda_{\ell+1}$.

Conjecture 1B. The Appendix $C$ construction produces an injection Я : $\Lambda_{\ell} \longrightarrow$ $\Lambda_{\ell+1}$.

Conjecture 1C. The Appendix $C$ construction produces a monomorphism Я : $\Lambda_{\ell} \longrightarrow \Lambda_{\ell+1}$ that respects the action of the ambient groups $\Lambda_{\ell}$ and $\Lambda_{\ell+1}$ on the sets $n$ the sets $\mathbb{K}^{(\ell)}$ and $\mathbb{K}^{(\ell+1)}$. 


\section{Lattice knot type}

We are now finally at a point where we can define lattice knot type.

Definition 13.1. Two lattice knots $K_{1}$ and $K_{2}$ of order $\ell$ are said to be of the same knot $\ell$-type, written

$$
K_{1} \underset{\ell}{\sim} K_{2}
$$

provided there exists an element $g$ of the lattice ambient group $\Lambda_{\ell}$ that transforms one into the other. They are said to be of the same knot type, written

$$
K_{1} \sim K_{2}
$$

provided there exists a non-negative integer $m$ such that

$$
\boldsymbol{Я}^{m} K_{1} \underset{\ell+m}{\sim} \boldsymbol{Я ~}^{m} K_{2}
$$

In like manner, we can define inextensible knot $\ell$-type and inextensible knot type as follows:

Definition 13.2. Two lattice knots $K_{1}$ and $K_{2}$ of order $\ell$ are said to be of the same inextensible knot $\ell$-type, written

$$
K_{1} \underset{\ell}{\approx} K_{2}
$$

provided there exists an element $g$ of the inextensible lattice ambient group $\widetilde{\Lambda}_{\ell}$ that transforms one into the other. They are said to be of the same inextensible knot type, written

$$
K_{1} \approx K_{2}
$$

provided there exists a non-negative integer $m$ such that

$$
\boldsymbol{Я}^{m} K_{1} \underset{\ell+m}{\approx} \boldsymbol{Я}^{m} K_{2}
$$

Because the move tug is part of the definition of knot $\ell$-type, but not part of the definition of inextensible knot $\ell$-type, we have the following proposition:

\section{The preferred vertex (PV) approximation for knots}

Definition 14.1. A knot $x$ in Euclidean 3 -space $\mathbb{R}^{3}$ is said to be finitely piecewise smooth (FPS) provided the knot $x$ consists of finitely many smooth $\left(C^{\infty}\right)$ segments $x_{1}, x_{2}, \ldots, x_{r}$ with no two segments tangent to each other at their endpoints $^{3}$. Such a knot $x$ will be called an FPS knot. Obviously, every lattice knot is an FPS knot.

\footnotetext{
${ }^{3}$ We should also mention that we really only need for the curve $x$ to be piecewise $C^{3}$.
} 
In this section, we will create, for each non-negative integer $\ell$, a procedure for finding an $\ell$-th order lattice graph $P V^{(\ell)}(x)$, called the $\ell$-th preferred vertex approximation, that approximates an arbitrary FPS knot $x$ in $\mathbb{R}^{3}$.

We begin by noting that, for each non-negative integer $\ell$, the set

$$
\left\{B^{(\ell)}\left(a_{\beta}\right) \cup\left(\bigcup_{p=1}^{3} F_{p}^{(\ell)}\left(a_{\beta}\right)\right) \cup\left(\bigcup_{p=1}^{3} E_{p}^{(\ell)}\left(a_{\beta}\right)\right) \cup\{a\}=\overline{B^{(\ell)}\left(a_{\beta}\right)}-\bigcup_{p=1}^{3} \overline{F_{p}^{(\ell)}\left(a_{\beta}^{: p}\right)} \quad: \quad a \in \mathcal{L}_{\ell}\right\}
$$

of half closed cubes is a partition of 3 -space $\mathbb{R}^{3}$. (We have used $\bar{C}$ to denote the closure of a cell $C$.) So we can create a map of space $\mathbb{R}^{3}$ into the lattice $\mathcal{L}_{\ell}$ simply by mapping each point of a half closed cube to the preferred vertex of that half closed cube ${ }^{4}$ :

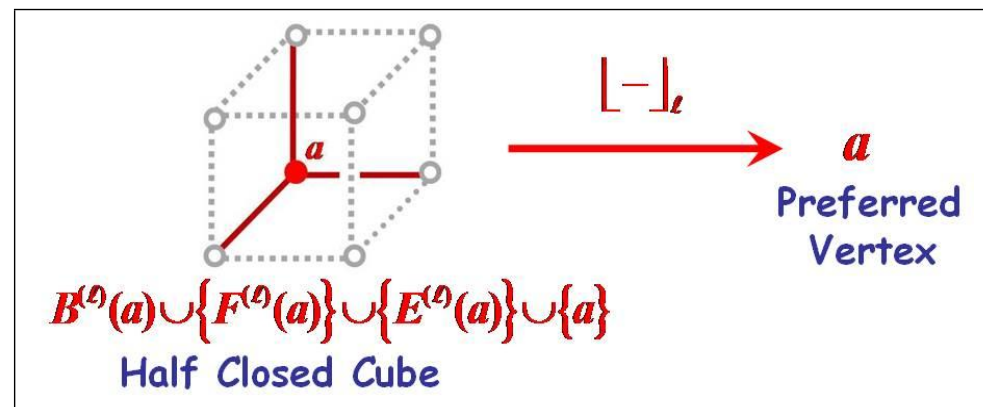

\section{The preferred vertex map which maps each half closed cube to its preferred vertex.}

Definition 14.2. We define the $\ell$-th preferred vertex map $\lfloor-\rfloor_{\ell}: \mathbb{R}^{3} \longrightarrow \mathcal{L}_{\ell}$ from Euclidean 3 -space $\mathbb{R}^{3}$ to the lattice $\mathcal{L}_{\ell}$ as

$$
\begin{aligned}
& \lfloor-\rfloor_{\ell}: \quad \mathbb{R}^{3} \quad \longrightarrow \quad \mathcal{L}_{\ell} \\
& x=\left(x_{1}, x_{2}, x_{3}\right) \longmapsto\lfloor x\rfloor_{\ell}=2^{-\ell}\left\lfloor 2^{\ell} x\right\rfloor=\left(2^{-\ell}\left\lfloor 2^{\ell} x_{1}\right\rfloor, 2^{-\ell}\left\lfloor 2^{\ell} x_{2}\right\rfloor, 2^{-\ell}\left\lfloor 2^{\ell} x_{3}\right\rfloor\right)
\end{aligned}
$$

where $\lfloor-\rfloor$ denotes the floor function.

REMARK 14.3. It immediately follows that the inverse image under $\lfloor-\rfloor_{\ell}$ of each vertex $a$ in the lattice $\mathcal{L}_{\ell}$ is the half closed cube $\overline{B^{(\ell)}}(a)-\bigcup_{p=1}^{3} \overline{F_{p}^{(\ell)}}\left(a^{: p}\right)$.

REMARK 14.4. Let $r$ be a real number. Then $\lfloor r\rfloor_{\ell}$ is the rational number resulting from deleting, in the binary expansion of $r$, all bits to the right of the $\ell$-th bit after the decimal point. For example, $\left\lfloor(1101.10110100 \ldots)_{2}\right\rfloor_{5}=(1101.10110)_{2}$, where $(-)_{2}$ denotes the binary expansion of a real number.

\footnotetext{
${ }^{4}$ We remind the reader that cells $E_{p}^{(\ell)}\left(a_{\beta}\right), F_{p}^{(\ell)}\left(a_{\beta}\right), B^{(\ell)}\left(a_{\beta}\right)$ are open cells, unless stated otherwise.
} 
Let $\ell$ be an arbitrarily chosen non-negative integer. Let $x$ be an arbitrary FPS knot in $\mathbb{R}^{3}$, and let $x_{1}, x_{2}, \ldots, x_{r}$ denote the simple closed FPS curves which are the components of $x$. (Thus each component $x_{\beta}$ consists of finitely many piecewise smooth segments.)

For each component $x_{\beta}$, choose any one of the two possible orientations. Also select an arbitrary point $P_{0, \beta}$ of $x_{\beta}$. Starting at the point $P_{0, \beta}$, traverse the curve $x_{\beta}$ in the direction of its orientation all the way around from $P_{0, \beta}$ back to $P_{0, \beta}$. As the simple closed curve is traversed, the preferred vertex map $[-\rfloor_{\ell}$ will produce a finite sequence of lattice points

$$
a_{\beta}^{(0)}, a_{\beta}^{(1)}, a_{\beta}^{(2)}, \ldots, a_{\beta}^{\left(u_{\beta}\right)}=a_{\beta}^{(0)}
$$

For each pair of two consecutive vertices $\left(a_{\beta}^{(j)}, a_{\beta}^{(j+1)}\right)$ of this sequence, select a shortest path from vertex $a_{\beta}^{(j)}$ to vertex $a_{\beta}^{(j+1)}$ that lies in the 1 -skeleton $\mathcal{C}_{\ell}^{1}$ of the cell complex $\mathcal{C}_{\ell}$, and let $S_{j, \beta}$ denote the set of edges and vertices in the selected shortest path. An $\ell$-th order preferred vertex approximation of the knot component $x_{\beta}$, written $P V^{(\ell)}\left(x_{\beta}\right)$, is defined as the lattice graph

$$
P V^{(\ell)}\left(x_{\beta}\right)=\bigcup_{j=0}^{u_{\beta}-1} S_{j, \beta}
$$

Finally, an $\ell$-th order preferred vertex approximation of the link $x$, written $P V^{(\ell)}(x)$, is defined as the lattice graph

$$
P V^{(\ell)}(x)=\bigcup_{\beta=0}^{r} P V^{(\ell)}\left(x_{\beta}\right)
$$

The following lemma is a consequence of the observation that, since the knot $x$ is piecewise smooth and compact, its curvature and torsion are bounded.

LEMmA 14.5. Let $x$ be a FPS knot in Euclidean 3-space $\mathbb{R}^{3}$. Then there exists a non-negative integer $\ell_{0}^{\prime}=\ell_{0}^{\prime}(x)$ such that, for $\ell \geq \ell_{0}^{\prime}$, every $\ell$-th order preferred vertex approximation $P V^{(\ell)}(x)$ of $x$ is a lattice knot of order $\ell$. In other words, for sufficiently large $\ell, P V^{(\ell)}(x)$ of $x$ is an $\ell$-th order lattice knot.

COROLLARY 2. Let $x$ be a FPS knot in Euclidean 3-space $\mathbb{R}^{3}$, and let $\epsilon$ be an arbitrary positive real number. Then there exists a non-negative integer $\ell_{0}=\ell_{0}(x, \epsilon)$ such that, for $\ell \geq \ell_{0}$, every $\ell$-th order preferred vertex approximation $P V^{(\ell)}(x)$ of $x$ is such that

1) $P V^{(\ell)}(x)$ is a lattice knot of order $\ell$,

2) $P V^{(\ell)}(x)$ lies inside the open tubular neighborhood of $x$ of radius $\epsilon$, and

3) $P V^{(\ell)}(x)$ is of the same knot type as the knot $x$. 
REMARK 14.6. The key feature of the preferred vertex approximation is that there exists a non-negative integer $\ell_{0}$ such that $P V^{(\ell)}(x)$ is of the same knot knot type as the knot $x$. The reader should note, however, that the length of $P V^{(\ell)}(x)$ is is the same for all $\ell \geq 0$. Hence, the $\operatorname{limit}_{\ell \rightarrow \infty} P \lim ^{(\ell)}(x)$, if it exists, is not necessarily the knot $x$. An illustration of this is the unit circle

$$
\left\{x \in \mathbb{R}^{3}: x_{1}^{2}+x_{2}^{2}=1, x_{3}=0\right\} .
$$

While the circle $x$ is of total length $2 \pi$, the total length of its preferred vertex approximation $P V^{(\ell)}(x)$ is 8 for all $\ell \geq 0$. Thus, for sufficiently large $\ell$, the preferred vertex approximation $P V^{(\ell)}(x)$ can be thought of as a "wrinkled up" version of the original knot $x$.

\section{Wiggle, Wag, and Tug variational derivatives, and knot invariants}

In this section, we will define wiggle, wag, and tug variational derivatives, and discuss how they are connected to knot invariants. We begin by defining a domain for these variational derivatives. We will be making extensive use of the calculus of variation. For readers unfamiliar with this subject, we refer them, for example, to $[\mathbf{1 4}]$.

DEFINITION 15.1. Let $\mathfrak{K}$ be the topological space ${ }^{5}$ of all finitely piecewise smooth (FPS) knots $^{6}$ with the compact-open topology. A subspace $\mathfrak{K}^{\prime}$ of $\mathfrak{K}$ is said to be FPS-complete subspace of $\mathfrak{K}$ provided for every knot $x^{\prime}$ in $\mathfrak{K}^{\prime}$ and for every knot $x$ in $\mathfrak{K}, x^{\prime}$ is of the same knot type as $x$ (written $x^{\prime} \sim x$ ) implies that $x$ also lies in $\mathfrak{K}^{\prime}$.

REMARK 15.2. We should mention that the set of all lattice knots

$$
\mathbb{K}=\bigcup_{\ell=0}^{\infty} \mathbb{K}^{(\ell)} \subset \mathfrak{K}
$$

is a proper subset of the set $\mathfrak{K}$ of FPS knots, but is by no means an FPS-complete subspace of $\mathfrak{K}$.

ExAMPLE 15.3. An example of a FPS-complete subspace $\mathfrak{K}^{\prime}$ of $\mathfrak{K}$ would be the topological space $\mathcal{L} \mathcal{I} \mathcal{N} \mathcal{K}_{2}$ of all FPS knots which are two component links, i.e.,

$$
\mathcal{L} \mathcal{I N} \mathcal{K}_{2}=\{x \in \mathfrak{K}: x \text { is a two component link }\}
$$

DEFinition 15.4. A real valued map $F: \mathfrak{K}^{\prime} \longrightarrow \mathbb{R}$ on a FPS-complete subspace $\mathfrak{K}^{\prime}$ of $\mathfrak{K}$ in will be called a knot functional.

\footnotetext{
${ }^{5}$ Actualy, this space has much more mathematical structure. But to use this additional structure would take us far beyond the scope of this paper.

${ }^{6}$ We remind the reader that in this paper "knot" means either a knot or a link.
} 
EXAMPLE 15.5. Let $x=\sqcup_{\beta=1}^{r} K_{\beta}$ be an $r$-component knot in $\mathfrak{K}$, i.e., $x$ is the disjoint union of $r$ simple closed FPS curves $x_{1}, x_{2}, \ldots, x_{r}$. For each component $x_{\beta}$, let $x_{\beta}=x_{\beta}\left(s_{\beta}\right)=\left(x_{\beta, 1}, x_{\beta, 2}, x_{\beta, 3}\right)$ be a parameterization by arclength $s_{\beta}$, $0 \leq s_{\beta} \leq L_{\beta}$, where $L_{\beta}$ denotes the length of $x_{\beta}$. Then the following is an example of a knot functional which is an invariant of inextensible knot type.

$$
\begin{aligned}
\text { Length: } \mathfrak{K} & \longrightarrow \mathbb{R} \\
x & \longmapsto \sum_{\beta=1}^{r} \oint \sqrt{\left(\frac{d x_{\beta, 1}}{d s_{\beta}}\right)^{2}+\left(\frac{d x_{\beta, 2}}{d s_{\beta}}\right)^{2}+\left(\frac{d x_{\beta, 3}}{d s_{\beta}}\right)^{2}} d s_{\beta}
\end{aligned}
$$

EXAMPLE 15.6. An example of a knot functional, which is an invariant of knot type, is the magnitude of the Gauss integral. Let $x$ be an arbitrary knot in $\mathcal{L} \mathcal{I N} \mathcal{K}_{\mathbf{2}}$, and let $\xi$ and $\zeta$ denote its two components. Then the magnitude

$$
\operatorname{Link}[x]=\frac{1}{4 \pi}\left|\oint_{\xi} \oint_{\zeta} \frac{(\zeta-\xi) \times d \zeta}{|\zeta-\xi|^{3}} \cdot d \xi\right|
$$

of the Gauss integral of $x$ is an invariant of inextensible knot type, where $\xi$ and $\zeta$ denote its two components ${ }^{7}$.

We will need the following elements of the ambient group $\Lambda_{\ell}$ :

- $1{ }^{(\ell)}(a, p)=\prod_{q=0}^{3} L_{1}^{(\ell)}(a, p, q)$, called a total tug on the face $F_{p}^{(\ell)}(a), p=$ $1,2,3$

- $2^{(\ell)}(a, p)=\prod_{q=0}^{1} L_{2}^{(\ell)}(a, p, q)$, called a total wiggle on the face $F_{p}^{(\ell)}(a)$, $p=1,2,3$

- $3^{(\ell)}(a, p)=\prod_{q=0}^{3} L_{3}^{(\ell)}(a, p, q)$, called a total wag on the face $F_{p}^{(\ell)}(a), p=$ $1,2,3$

REMARK 15.7. The reader should note that all the elements in each of the above products commute with one another. Moreover, at most one element in each product can be different from the identity transformation when the total move is applied to a specific lattice knot $K$. For example, in the product for the total tug $1^{(\ell)}(a, p)$, the tugs $L_{1}^{(\ell)}(a, p, 0), L_{1}^{(\ell)}(a, p, 1), L_{1}^{(\ell)}(a, p, 2), L_{1}^{(\ell)}(a, p, 3)$ all commute with one another, and moreover, when they are applied to a specific lattice knot, at most one of these tugs is different from the identity 1.

We are now in a position to define wiggle, wag, and tug variational derivatives.

\footnotetext{
${ }^{7}$ We use the absolute value of the Gauss integral since only unoriented knots and links are discussed in this paper. Everything in this paper can easily be extended to oriented knots. With that generalization, there is no longer a need to take the magnitude of the Gauss integral.
} 
DEFINITION 15.8. Let $\mathfrak{K}^{\prime}$ be a FPS-complete subspace of the space $\mathfrak{K}$ of all FPS knots, and let $x$ be a FPS knot in $\mathfrak{K}^{\prime}$. Moreover, let

$$
F: \mathfrak{K}^{\prime} \longrightarrow \mathbb{R}
$$

be a knot functional. At each point $a \in x$, we define the tug, wiggle, and wag variational derivatives respectively as follows:

- $\frac{\delta F[x]}{\delta[1(a, p)}=\lim _{\ell \rightarrow \infty} \frac{F\left[{ }^{\left.(\ell]^{(\ell)}(a, p) P V^{(\ell)}(x)\right]-F\left[P V^{(\ell)}(x)\right]}\right.}{\left(^{-\ell}\right)^{2}}$, whenever the limit ex- $\frac{\delta F[x]}{\delta[2[a, p)}=\lim _{\ell \rightarrow \infty} \frac{F\left[[2]^{(\ell)}(a, p) P V^{(\ell)}(x)\right]-F\left[P V^{(\ell)}(x)\right]}{\left(2^{-\ell}\right)^{2}}$, whenever the limit ex- $\frac{\delta F[x]}{\delta[3(a, p)}=\lim _{\ell \rightarrow \infty} \frac{F\left[[]^{(\ell)}(a, p) P V^{(\ell)}(x)\right]-F\left[P V^{(\ell)}(x)\right]}{\left(^{-\ell}\right)^{2}}$, whenever the limit exfor $p=1,2,3$, where $\left(2^{-\ell}\right)^{2}$ is the area of the face $F_{p}^{(\ell)}(a)$. Moreover, we define the tug, wiggle, and wag variational gradients as

$$
\frac{\delta F[K]}{\delta m(a)}=\left(\frac{\delta F[K]}{\delta[m(a, 1)}, \frac{\delta F[K]}{\delta[m(a, 2)}, \frac{\delta F[K]}{\delta[m(a, 3)}\right) .
$$

ConjeCture 1. A functional $F: \mathfrak{K}^{\prime} \longrightarrow \mathbb{R}$ is a knot invariant if all its tug, wiggle, and wag variational gradients exist and are equal to $(0,0,0)$. The functional $F$ is an inextensible knot invariant if all its wiggle, and wag variational gradients exist and are equal to $(0,0,0)$.

We leave the following two exercises for the reader:

Exercise 1. Show that for all knots $K$ in $\mathbb{K}^{(\infty)}=\bigcup_{\ell=0}^{\infty} \mathbb{K}^{(\ell)}$, the wiggle and wag variational gradients of the functional Length $[K]$ vanish at each vertex of $K$.

Exercise 2. Show that for all knots $K$ in $\mathbb{K}^{(\infty)} \cap \mathcal{L I N \mathcal { N }}{ }_{\mathbf{2}}$, the tug, wiggle, and wag variational gradients of the functional Link $[K]$ vanish at each vertex of $K$. 


\section{Infinitesimal Wiggles, Wags, and Tugs, differential forms, and integrals}

There are many consequences to the research developments discussed in the previous section of this paper.

For example, the approach found in the previous section lead to the construction of infinitesimal wiggles, wags, and tugs, such as for example the infinitesimal wiggle

$$
\left\ulcorner(x)^{\frac{\partial}{\partial x_{1}} \otimes \frac{\partial}{\partial x_{2}}}=\lim _{\ell \rightarrow \infty}\lceil\rfloor^{(\ell)}\left(\lfloor x\rfloor_{\ell}, 3\right) .\right.
$$

Moreover, there is also the corresponding differential form

$$
\text { Г }(x)^{d x_{1} d x_{2}},
$$

and its multiplicative integrals, such as for example,

$$
\int_{x_{1}=0 . .1} \int_{x_{2}=0 . .1}^{\rho} \Gamma_{(}\left(x_{1}, x_{2}, 0\right)^{d x_{1} d x_{2}}
$$

where, for example,

$$
\left.(\lrcorner^{(0)}((0,0,0), 3)\right)(K)=\left(\int_{x_{1}=0 . .1}^{\rho} \int_{x_{2}=0 . .1}^{\rho} \Gamma\left(x_{1}, x_{2}, 0\right)^{d x_{1} d x_{2}}\right)(K),
$$

for all 0 -th order lattice knots $K$ such that

$$
K \cap F_{3}^{(0)}((0,0,0))=\underset{ }{0} \text { or }
$$

It is because of these developments (which were motivated by $[\mathbf{2 6}]$ ) that we have suggested that the moves wiggle, wag, and tug are more "physics friendly" than the Reidemeister moves. Unfortunately, because of the scope of this current paper, this section is of necessity sketchy and abbreviated. Readers interested in a more in depth discussion of this material are referred to the upcoming paper [32].

\section{7. n-bounded lattice knots}

As a preliminary step to defining quantum knots and quantum knot systems, we will now put bounds on the mathematical constructs given in previous sections of this paper.

We define the $\mathbf{n}$-bounded lattice $\mathcal{L}_{\ell, n}$ as the sublattice of $\mathcal{L}_{\ell}$ given by

$$
\mathcal{L}_{\ell, n}=\left\{a \in \mathcal{L}_{\ell}: 0 \leq a_{j} \leq n, \text { for } j=1,2,3\right\} .
$$

Let $\mathcal{C}_{\ell, n}$ denote the corresponding $n$-bounded cell complex, and $\mathcal{C}_{\ell, n}^{j}$ its $n$ bounded $j$-skeleton. We also define a bounded lattice knot $K$ of order $(\ell, n)$ as a closed 2-valent subgraph of the $n$-bounded 1 -skeleton $\mathcal{C}_{\ell, n}^{1}$, and let $\mathbb{K}^{(\ell, n)}$ denote the set of all bounded lattice knots of order $(\ell, n)$. 
REMARK 17.1. For the sake of simplicity, we have defined $n$-bounded lattices that only lie in the first octant. From the perspective of this paper there is no need to extend the definition to all of $\mathbb{R}^{3}$. Such an extension would only lead to unnecessary additional complexity.

Definition 17.2. The bounded (lattice) ambient group $\Lambda_{\ell, n}$ (of order $(\ell, n))$ is the finite subgroup of the (lattice) ambient $\Lambda_{\ell}$ generated by all tugs $L_{1^{\prime}}^{(\ell)}(a, p, q)$ and wiggles $L_{2^{*}}^{(\ell)}(a, p, q)$ such that

$$
0 \leq a_{p} \leq n, \quad 0 \leq a_{\lfloor p} \leq n-1, \quad 0 \leq a_{p\rceil} \leq n-1
$$

and by all wags $L_{3^{*}}^{(\ell)}(a, p, q)$ such that $0 \leq a_{j} \leq n-1$, for $j=1,2,3$.

We will also have need of the lattice knot injection defined by

$$
\begin{aligned}
\iota: \mathbb{K}^{(\ell, n)} & \longrightarrow \mathbb{K}^{(\ell, n+1)} \\
K^{(\ell, n)} & \longmapsto K^{(\ell, n+1)},
\end{aligned}
$$

where $K^{(\ell, n+1)}=\iota\left(K^{(\ell, n)}\right)$ is the lattice knot of order $(\ell, n+1)$ consisting of all the edges in $K^{(\ell, n)}$. Moreover, we define in the obvious way the (lattice) knot group monomorphism

$$
\iota: \Lambda_{\ell, n} \longrightarrow \Lambda_{\ell, n+1}
$$

Definition 17.3. Let $\mathbb{K}^{[\ell]}$ denote the directed system of bounded lattice knots $\left\{\mathbb{K}^{(\ell, n)} \longrightarrow \mathbb{K}^{(\ell, n+1)}: n=1,2,3, \ldots\right\}$ and $\Lambda_{[\ell]}$ the directed system of bounded ambient groups $\left\{\Lambda_{\ell, n} \longrightarrow \Lambda_{\ell, n+1}: n=1,2,3, \ldots\right\}$. Finally,let $\left(\mathbb{K}^{[\ell]}, \Lambda_{[\ell]}\right)$ denote the directed graded system

$$
\left(\mathbb{K}^{[\ell]}, \Lambda_{[\ell]}\right)=\left(\mathbb{K}^{(\ell, 1)}, \Lambda_{\ell, 1}\right) \longrightarrow\left(\mathbb{K}^{(\ell, 2)}, \Lambda_{\ell, 2}\right) \longrightarrow \cdots \longrightarrow\left(\mathbb{K}^{(\ell, n)}, \Lambda_{\ell, n}\right) \longrightarrow \cdots
$$

Definition 17.4. Two lattice knots $K_{1}$ and $K_{2}$ of order $(\ell, n)$ are said to be of the same bounded knot $(\ell, n)$-type, written

$$
K_{1} \underset{\ell}{\stackrel{n}{\sim}} K_{2}
$$

provided there exists an element $g$ of the bounded ambient group $\Lambda_{\ell, n}$ that transforms one into the other. They are said to be of the same knot $(\ell, \infty)$-type, written

$$
K_{1} \underset{\ell}{\stackrel{\infty}{\ell}} K_{2}
$$

provided there exists a non-negative integer $n^{\prime}$ such that

$$
\iota^{n^{\prime}} K_{1} \underset{\ell}{\stackrel{n+n^{\prime}}{\sim}} \iota^{n^{\prime}} K_{2}
$$

There are of the same knot $(\infty, n)$-type, written

$$
K_{1} \underset{\infty}{\stackrel{n}{\sim}} K_{2}
$$

provided there exists a non-negative integer $\ell^{\prime}$ such that

$$
\boldsymbol{g}^{\ell^{\prime}} K_{1} \underset{\ell+\ell^{\prime}}{\stackrel{n}{\sim}} \boldsymbol{9}^{\ell^{\prime}} K_{2}
$$


It immediately follows that:

Proposition 4. $K_{1} \underset{\ell}{\stackrel{\infty}{\sim}} K_{2} \Longleftrightarrow K_{1} \underset{\infty}{\stackrel{n}{\sim}} K_{2} \Longleftrightarrow K_{1} \sim K_{2}$

In like manner, we can define inextensible knot $(\ell, n)$-type and inextensible knot type.

Definition 17.5. Two lattice knots $K_{1}$ and $K_{2}$ of order $(\ell, n)$ are said to be of the same inextensible knot $(\ell, n)$-type, written

$$
K_{1} \underset{\ell}{\stackrel{n}{\approx}} K_{2}
$$

provided there exists an element $g$ of the bounded inextensible ambient group $\widetilde{\Lambda}_{\ell, n}$ that transforms one into the other. They are said to be of the same inextensible knot $(\ell, \infty)$-type, written

$$
K_{1} \underset{\ell}{\stackrel{\infty}{\approx}} K_{2}
$$

provided there exists a non-negative integer $n^{\prime}$ such that

$$
\iota^{n^{\prime}} K_{1} \underset{\ell}{\stackrel{n+n^{\prime}}{\approx}} \iota^{n^{\prime}} K_{2}
$$

They are of the same inextensible knot $(\infty, n)$-type, written

$$
K_{1} \underset{\infty}{\stackrel{n}{\approx}} K_{2}
$$

provided there exists a non-negative integer $\ell^{\prime}$ such that

$$
\boldsymbol{9}^{\ell^{\prime}} K_{1} \underset{\ell+\ell^{\prime}}{\stackrel{n}{\approx}} \boldsymbol{9}^{\ell^{\prime}} K_{2}
$$

Proposition 5. $K_{1} \underset{\infty}{\stackrel{n}{\approx}} K_{2} \Longleftrightarrow K_{1} \approx K_{2}$. But $K_{1} \underset{\ell}{\approx} \approx K_{2} \nRightarrow K_{1} \approx K_{2}$.

\section{Part 2. Quantum Knots}

We are finally ready to define what is meant by a quantum knot system and a quantum knot. 


\section{The Definition of a Quantum Knot}

We will now create a Hilbert space by associating a qubit with each edge of the cell complex $\mathcal{C}_{\ell, n}$.

Let ' $<$ ' denote the lexicographic (lex) ordering of the lattice points $\mathcal{L}_{\ell, n}$ induced by the standard linear ordering of the rationals. Extend this ordering in the obvious way to a lex ordering of $\mathcal{L}_{\ell, n} \times\{1,2,3\}$, also denoted by ' $<$ '. Finally, define a linear ordering, again denoted by ' $<$ ' on the set $\mathcal{E}_{\ell, n}$ of edges of the cell complex $\mathcal{C}_{\ell, n}$ given by

$$
E_{p}(a)<E_{p^{\prime}}\left(a^{\prime}\right) \text { if and only if }(a, p)<\left(a^{\prime}, p^{\prime}\right) .
$$

Let $\mathcal{H}$ be the two dimensional Hilbert space (called the edge state space) with orthonormal basis

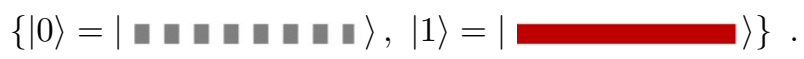

The Hilbert space $\mathcal{G}_{\ell, n}$ of lattice graphs of order $(\ell, n)$ is defined as the tensor product

$$
\mathcal{G}_{\ell, n}=\bigotimes_{E \in \mathcal{E}_{\ell, n}} \mathcal{H}
$$

where the tensor product is taken with respect to the above defined linear ordering ' $<$ '. Thus, as orthonormal basis for the Hilbert space $\mathcal{G}_{\ell, n}$, we have

$$
\left\{\underset{E \in \mathcal{E}_{\ell, n}}{\otimes}|c(E)\rangle: c \in \operatorname{Map}\left(\mathcal{E}_{\ell, n},\{0,1\}\right)\right\},
$$

where $\operatorname{Map}\left(\mathcal{E}_{\ell, n},\{0,1\}\right)$ is the set of all maps $c: \mathcal{E}_{\ell, n} \longrightarrow\{0,1\}$ from the set $\mathcal{E}_{\ell, n}$ of edges to the set $\{0,1\}$.

We identify in the obvious way each basis element

$$
\underset{E \in \mathcal{E}_{\ell, n}}{\otimes}|c(E)\rangle
$$

with a corresponding lattice graph $G$. Under this identification, the space $\mathcal{G}_{\ell, n}$ becomes the Hilbert space with orthonormal basis

$$
\left\{|G\rangle: G \text { a lattice graph in } \mathcal{L}_{\ell, n}\right\},
$$

called the standard basis. Finally, the Hilbert space $\mathcal{K}^{(\ell, n)}$ of lattice knots of order $(\ell, n)$ is defined as the sub-Hilbert space of $\mathcal{G}_{\ell, n}$ with orthonormal basis

$$
\left\{|K\rangle: K \in \mathbb{K}^{(\ell, n)}\right\}
$$

Our next step is to identify each element $g$ of the ambient group $\Lambda_{\ell, n}$ with the corresponding linear transformation defined by

$$
\begin{array}{ccc}
\mathcal{K}^{(\ell, n)} & \stackrel{g}{ } \mathcal{K}^{(\ell, n)} \\
|K\rangle & \longmapsto & |g K\rangle
\end{array}
$$

This is a unitary transformation, since each element $g$ simply permutes the basis elements of $\mathcal{K}^{(\ell, n)}$. In this way, the ambient group $\Lambda_{\ell, n}$ is identified with the discrete unitary subgroup (also denoted by $\Lambda_{\ell, n}$ ) of the group $U\left(\mathcal{K}^{(\ell, n)}\right)$, where $U\left(\mathcal{K}^{(\ell, n)}\right.$ ) 
denotes the group of all unitary transformations on the Hilbert space $\mathcal{K}^{(\ell, n)}$. We also call the unitary group $\Lambda_{\ell, n}$ the (lattice) ambient group of order $(\ell, n)$.

$|K\rangle$
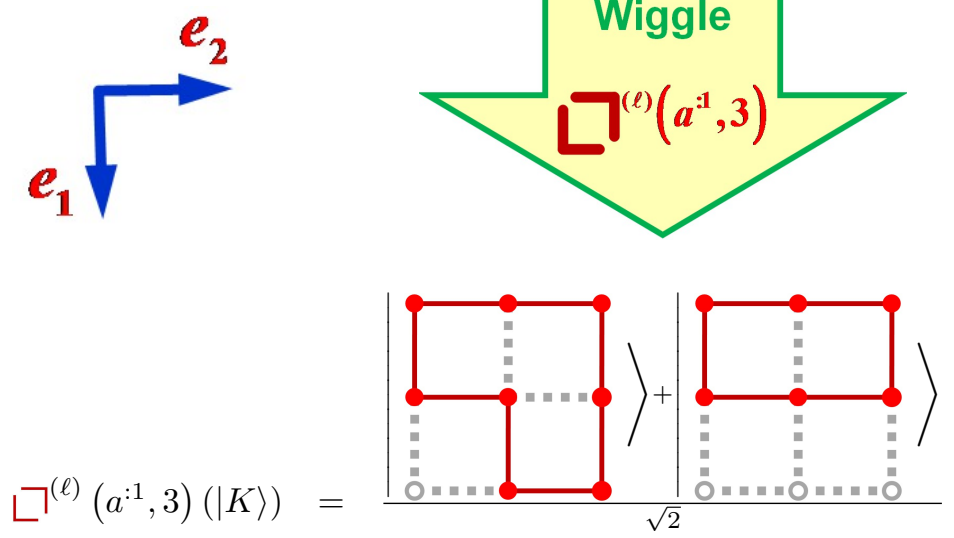

An example of the action of the ambient group $\Lambda_{\ell, n}$ on a quantum knot in $\mathcal{K}^{(\ell, n)}$.

We leave, as an exercise for the reader, the definition of the (lattice) inextensible ambient group $\widetilde{\Lambda}_{\ell, n}$ of order $(\ell, n)$, which is defined in like manner.

Finally, everything comes together with the following definition.

Definition 19.1. Let $\ell \geq 0$ and $n \geq 1$ be a integers. A quantum knot system $Q\left(\mathcal{K}^{(\ell, n)}, \Lambda_{\ell, n}\right)$ of order $(\ell, n)$ is a quantum system with the Hilbert space $\mathcal{K}^{(\ell, n)}$ of $(\ell, n)$-th order lattice knots as its state space, having the ambient group $\Lambda_{\ell, n}$ as an accessible unitary control group. The states of the quantum system $Q\left(\mathcal{K}^{(\ell, n)}, \Lambda_{\ell, n}\right)$ are called quantum knots of order $(\ell, n)$, and the elements of the ambient group $\Lambda_{\ell, n}$ are called unitary knot moves. Moreover, the quantum knot system $Q\left(\mathcal{K}^{(\ell, n)}, \Lambda_{\ell, n}\right)$ is a subsystem of the quantum knot system $Q\left(\mathcal{K}^{(\ell, n+1)}, \Lambda_{\ell, n+1}\right)$. Thus, the quantum knot systems $Q\left(\mathcal{K}^{(\ell, n)}, \Lambda_{\ell, n}\right)$ collectively become a nested sequence of quantum knot systems

$Q\left(\mathcal{K}^{(\ell)}, \Lambda_{\ell}\right)=Q\left(\mathcal{K}^{(\ell, 1)}, \Lambda_{\ell, 1}\right) \longrightarrow \cdots \longrightarrow Q\left(\mathcal{K}^{(\ell, n)}, \Lambda_{\ell, n}\right) \longrightarrow Q\left(\mathcal{K}^{(\ell, n+1)}, \Lambda_{\ell, n}\right) \longrightarrow \cdots$

which we will denote simply by $Q\left(\mathcal{K}^{(\ell)}, \Lambda_{\ell}\right)$. We leave, as an exercise for the reader, the definition of the inextensible quantum knot system $Q\left(\mathcal{K}^{(\ell, n)}, \widetilde{\Lambda}_{\ell, n}\right)$ of order $(\ell, n)$, which is defined in like manner. 
REMARK 19.2. The nested quantum knot systems $Q\left(\mathcal{K}^{(\ell)}, \Lambda_{\ell}\right)$ and $Q\left(\mathcal{K}^{(\ell)}, \widetilde{\Lambda}_{\ell}\right)$ are probably not physically realizable systems. However, each quantum knot system $Q\left(\mathcal{K}^{(\ell, n)}, \Lambda_{\ell, n}\right)$ of order $(\ell, n)$ (as well as each inextensible quantum knot system $Q\left(\mathcal{K}^{(\ell, n)}, \widetilde{\Lambda}_{\ell, n}\right)$ of order $\left.(\ell, n)\right)$ is physically realizable. By this we mean that such quantum knot systems are physically realizable in the same sense as a quantum system implementing Shor's quantum factoring algorithm is physically realizable. $^{8}$

\section{Quantum knot type}

When are two quantum knots the same?

DEFinition 20.1. Let $\left|\psi_{1}\right\rangle$ and $\left|\psi_{2}\right\rangle$ be two quantum knots of a quantum knot system $Q\left(\mathcal{K}^{(\ell, n)}, \Lambda_{\ell, n}\right)$ (of an inextensible quantum knot system $Q\left(\mathcal{K}^{(\ell, n)}, \widetilde{\Lambda}_{\ell, n}\right)$ ). Then $\left|\psi_{1}\right\rangle$ and $\left|\psi_{2}\right\rangle$ are said to be of the same quantum knot $(\ell, n)$-type (of the same inextensible quantum knot $(\ell, n)$-type), written

$$
\left|\psi_{1}\right\rangle \underset{\ell}{\stackrel{n}{\sim}}\left|\psi_{2}\right\rangle,\left(\left|\psi_{1}\right\rangle \underset{\ell}{\stackrel{n}{\approx}}\left|\psi_{2}\right\rangle,\right)
$$

provided there exists a unitary transformation $g$ in the ambient group $\Lambda_{\ell, n}$ (in the inextensible ambient group $\left.\widetilde{\Lambda}_{\ell, n}\right)$ which transforms $\left|\psi_{1}\right\rangle$ into $\left|\psi_{2}\right\rangle$, i.e., such that

$$
g\left|\psi_{1}\right\rangle=\left|\psi_{2}\right\rangle .
$$

They are said to be of the same quantum knot type (of the same inextensible quantum knot type), written

$$
\left|\psi_{1}\right\rangle \sim\left|\psi_{2}\right\rangle,\left(\left|\psi_{1}\right\rangle \approx\left|\psi_{2}\right\rangle,\right)
$$

provided that for some non-negative integer $\ell^{\prime}$,

$$
\boldsymbol{Я}^{\ell^{\prime}}\left|\psi_{1}\right\rangle \underset{\ell+\ell^{\prime}}{\stackrel{n}{\sim}} \boldsymbol{Я}^{\ell^{\prime}}\left|\psi_{2}\right\rangle,\left(\boldsymbol{Я}^{\ell^{\prime}}\left|\psi_{1}\right\rangle \underset{\ell+\ell^{\prime}}{\stackrel{n}{\approx}} \boldsymbol{Я}^{\ell^{\prime}}\left|\psi_{2}\right\rangle,\right)
$$

where $\boldsymbol{Я}: \mathcal{K}^{(\ell, m)} \longrightarrow \mathcal{K}^{(\ell+1, m)}$ is the Hilbert space monomorphism induced by the previously defined refinement injection $\mathscr{Я}: \mathbb{K}^{(\ell, m)} \longrightarrow \mathbb{K}^{(\ell+1, m)}$.

Proposition 6. Let $\left|\psi_{1}\right\rangle$ and $\left|\psi_{2}\right\rangle$ be two quantum knots of a quantum system $Q\left(\mathcal{K}^{(\ell, n)}, \Lambda_{\ell, n}\right)$. Then $\left|\psi_{1}\right\rangle$ and $\left|\psi_{2}\right\rangle$ are of the same quantum knot type

$$
\left|\psi_{1}\right\rangle \sim\left|\psi_{2}\right\rangle
$$

if and only if there exists a non-negative integer $n^{\prime}$ such that

$$
\iota^{n^{\prime}}\left|\psi_{1}\right\rangle \underset{\ell}{\stackrel{n+n^{\prime}}{\sim}} \iota^{n^{\prime}}\left|\psi_{2}\right\rangle
$$

\footnotetext{
${ }^{8}$ It should be mentioned that, although the quantum knot systems $Q\left(\mathcal{K}^{(\ell, n)}, \Lambda_{\ell, n}\right)$ and $Q\left(\mathcal{K}^{(\ell, n)}, \widetilde{\Lambda}_{\ell, n}\right)$ are physically realizable, they may not be implementable with today's existing technology.
} 
where $\iota: \mathcal{K}^{(\ell, m)} \longrightarrow \mathcal{K}^{(\ell, m+1)}$ is the monomorphism induced by the previously defined injection $\iota: \mathbb{K}^{(\ell, m)} \longrightarrow \mathbb{K}^{(\ell, m+1)}$. The analogous statement for inextensible quantum knot type is false.

Thus, the two quantum knots found in the last example of the previous section are of the same quantum knot type. Surprisingly, the following two quantum knots $\left|\psi_{1}\right\rangle$ and $\left|\psi_{2}\right\rangle$ are neither of the same quantum knot $(1,1)$-type nor quantum knot type:

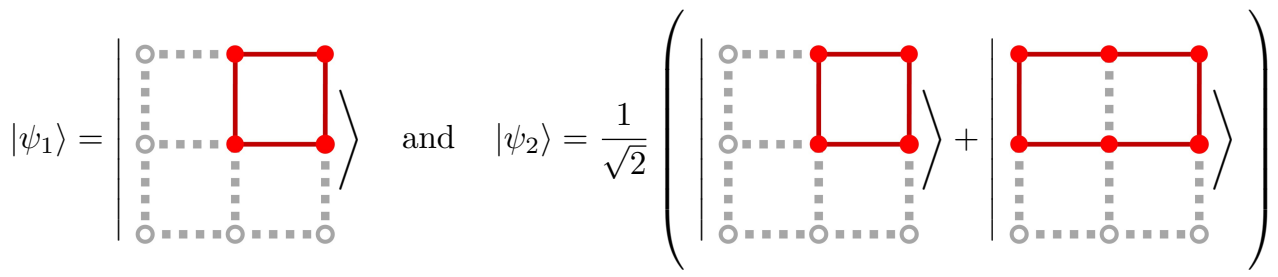

This follows from the fact that the ambient group $\Lambda_{\ell, n}$ is generated by a finite set of involutions.

\section{Hamiltonians of the generators of the ambient group $\Lambda$.}

In this section, we show how to find a Hamiltonians associated with the generators of the ambient group $\Lambda_{\ell, n}$, i.e., Hamiltonians associated with the wiggle, wag, and tug moves ${ }^{9}$.

Let $g$ be an arbitrary wiggle, wag, or tug move in the ambient group $\Lambda_{\ell, n}$. From proposition 1, we know that $g$, as a permutation, is the product of disjoint transpositions of knot $(\ell, n)$-th order lattice knots, i.e., of the form

$$
g=\left(K_{\alpha_{1}}, K_{\beta_{1}}\right)\left(K_{\alpha_{2}}, K_{\beta_{2}}\right) \cdots\left(K_{\alpha_{s}}, K_{\beta_{r}}\right)
$$

Without loss of generality, we can assume that $\alpha_{j}<\beta_{j}$ for $1 \leq j \leq r$, and that $\alpha_{j}<\alpha_{j+1}$ for $1 \leq j<r$, where ' $<$ ' is the lexicographic (lex) ordering on the set of $(\ell, n)$-lattice graphs $\mathcal{G}^{(\ell, n)}$ induced by the previously defined linear ordering ' $<$ ' of the edges in the lattice $\mathcal{L}_{\ell, n}$. For each permutation $\eta$ of $\mathcal{K}^{(\ell, n)}$, let ' $<_{\eta}$ ' denote the new linear ordering created by the application of the permutation $\eta$.

Choose a permutation $\eta$ such that

$$
K_{\alpha_{1}}<_{\eta} K_{\beta_{1}}<_{\eta} K_{\alpha_{2}}<_{\eta} K_{\beta_{2}}<_{\eta} \cdots<_{\eta} K_{\alpha_{r}}<_{\eta} K_{\beta_{r}}
$$

with $K_{\beta_{r}}<_{\eta}$ (i.e., $\eta$-less than) all other lattice knots of order $(\ell, n)$, and let $\sigma_{0}$ and $\sigma_{1}$ denote respectively the identity matrix and the first Pauli spin matrix given below

$$
\sigma_{0}=\left(\begin{array}{cc}
1 & 0 \\
0 & 1
\end{array}\right) \quad \text { and } \quad \sigma_{1}=\left(\begin{array}{cc}
0 & 1 \\
1 & 0
\end{array}\right)
$$

\footnotetext{
${ }^{9}$ Please keep in mind that the Hamiltonian construction found below is far from unique. There are many other ways of constructing a Hamiltonian corresponding to the generators of the ambient group $\Lambda_{\ell, n}$.
} 
Let $d(\ell, n)$ denote the dimension of the Hilbert space $\mathcal{K}^{(\ell, n)}$. Then in the $\eta$ reordered basis of the Hilbert space $\mathcal{K}^{(\ell, n)}$, the element $g$, as a unitary transformation, is of the form

$$
\eta^{-1} g \eta=\left(\begin{array}{ccccc}
\sigma_{1} & O & \ldots & O & O \\
O & \sigma_{1} & \ldots & O & O \\
\vdots & \vdots & \ddots & \vdots & \vdots \\
O & O & \ldots & \sigma_{1} & O \\
O & O & \ldots & O & I_{d(\ell, n)-2 r}
\end{array}\right)=\left(I_{r} \otimes \sigma_{1}\right) \oplus I_{d(\ell, n)-2 r},
$$

where ' $O$ ' denotes an all zero matrix of appropriate size, where $I_{d(\ell, n)-2 r}$ denotes the $(d(\ell, n)-2 r) \times(d(\ell, n)-2 r)$ identity matrix, and where ' $\oplus$ ' denotes the direct sum of matrices, i.e., $A \oplus B=\left(\begin{array}{cc}A & O \\ O & B\end{array}\right)$.

The natural $\log$ of $\sigma_{1}$ is

$$
\ln \sigma_{1}=\frac{i \pi}{2}(2 s+1)\left(\sigma_{0}-\sigma_{1}\right)
$$

where $s$ denotes an arbitrary integer. Hence, the natural $\log , \ln \left(\eta^{-1} g \eta\right)$, of the unitary transformation $\eta^{-1} g \eta$ is

$$
\frac{i \pi}{2}\left(\begin{array}{cccc}
\left(2 s_{1}+1\right)\left(\sigma_{0}-\sigma_{1}\right) & O & \cdots & O \\
O & \left(2 s_{2}+1\right)\left(\sigma_{0}-\sigma_{1}\right) & \cdots & O \\
\vdots & \vdots & \ddots & \vdots \\
O & O & \cdots & \left(2 s_{r}+1\right)\left(\sigma_{0}-\sigma_{1}\right)
\end{array}\right)
$$

where $D(\ell, n)=d(\ell, n)-2 r$, and where $s_{1}, s_{2}, \ldots, s_{r}$ are arbitrary integers. ${ }^{10}$

Since we are interested only in the simplest Hamiltonian, we choose the principal branch $\ln _{P}$ of the natural log, i.e., the branch for which $s_{1}=s_{2}=\cdots s_{r}=0$, and obtain for our Hamiltonian

$$
H_{g}=-i \eta\left[\ln _{P}\left(\eta^{-1} g \eta\right)\right] \eta^{-1}=\frac{\pi}{2} \eta\left(\begin{array}{cc}
I_{r} \otimes\left(\sigma_{0}-\sigma_{1}\right) & O \\
O & O_{(d(\ell, n)-2 r) \times(d(\ell, n)-2 r)}
\end{array}\right) \eta^{-1}
$$

Thus, if the initial quantum knot is

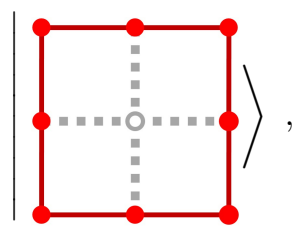

\footnotetext{
${ }^{10}$ Let $U$ be an arbitrary finite $r \times r$ unitary matrix, and let $W$ be a unitary matrix that diagonalizes $U$, i.e., a unitary matrix $W$ such that $W U W^{-1}=\Delta(\lambda(1), \lambda(2) \ldots, \lambda(r))$. Then the natural $\log$ of $A$ is $\ln A=W^{-1} \Delta(\ln \lambda(1), \ln \lambda(2) \ldots, \ln \lambda(r)) W$.
} 
and if we use the Hamiltonian $H_{g}$ for the wiggle move $\neg^{(0)}\left(a^{: 1}, 3\right)$, then the solution to Schroedinger's equation is

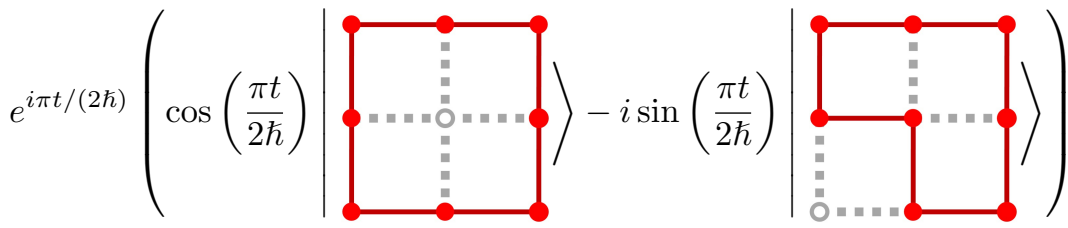

where $t$ denotes time, and where $\hbar$ denotes Planck's constant divided by $2 \pi$.

\section{Quantum observables as invariants of quantum knots}

We now consider the following question:

Question. What is a quantum knot invariant? How do we define it?

The objective of the first half of this section is to give a discursive argument that justifies a definition which will be found to be equivalent to the following:

$A$ quantum knot $(\ell, n)$-invariant for a quantum system $Q\left(\mathcal{K}^{(\ell, n)}, \Lambda_{\ell, n}\right)$ is an observable $\Omega$ on the Hilbert space $\mathcal{K}^{(\ell, n)}$ of quantum knots which is invariant under the action of the ambient group $\Lambda_{\ell, n}$, i.e., such that $U \Omega U^{-1}=\Omega$ for all $U$ in $\Lambda_{\ell, n}$.

Caveat: We emphasize to the reader that the above definition of quantum knot invariants is not the one currently used in quantum topology. Quantum topology uses analogies with quantum mechanics to create significant mathematical structures that do not necessarily correspond directly to quantum mechanical observables. The invariants of quantum topology have been investigated for their relevance to quantum computing and they can be regarded, in our context, as possible secondary calculations made on the basis of an observable. Here we are concerned with observables that are themselves topological invariants.

To justify our new use of the term 'quantum knot invariant,' we will use the following YARDSTICK:

YARDSTICK: Quantum knot invariants are to be physically meaningful invariants of quantum knot type. By "physically meaningful," we mean that the quantum knot invariants can be directly obtained from experimental data produced by an implementable physical experiment. ${ }^{11}$

Let $Q\left(\mathcal{K}^{(\ell, n)}, \Lambda_{\ell, n}\right)$ be a quantum knot system, where $\mathcal{K}^{(\ell, n)}$ is the Hilbert space of quantum knots, and where $\Lambda_{\ell, n}$ is the underlying ambient group on $\mathcal{K}^{(\ell, n)}$. Moreover, let $\mathcal{P}^{(\ell, n)}$ denote some yet-to-be-chosen mathematical domain. By an $(\ell, n)$-invariant $I^{(\ell, n)}$ of quantum knots, we mean a map

$$
I^{(\ell, n)}: \mathcal{K}^{(\ell, n)} \longrightarrow \mathcal{P}^{(\ell, n)},
$$

\footnotetext{
${ }^{11}$ Once again we remind the reader that, although the quantum knot system $Q\left(\mathcal{K}^{(\ell, n)}, \Lambda_{\ell, n}\right)$ is physically implementable, it may or may not be implentable within today's existing technology.
} 
such that, when two quantum knots $\left|\psi_{1}\right\rangle$ and $\left|\psi_{2}\right\rangle$ are of the same knot $(\ell, n)$-type, i.e., when

$$
\left|\psi_{1}\right\rangle \underset{\ell}{\stackrel{n}{\sim}}\left|\psi_{2}\right\rangle
$$

then their respective invariants must be equal, i.e.,

$$
I^{(\ell, n)}\left(\left|\psi_{1}\right\rangle\right)=I^{(\ell, n)}\left(\left|\psi_{2}\right\rangle\right)
$$

In other words, $I^{(\ell, n)}: \mathcal{K}^{(\ell, n)} \longrightarrow \mathcal{P}^{(\ell, n)}$ is a map which is invariant under the action of the ambient group $\Lambda_{\ell, n}$, i.e.,

$$
I^{(\ell, n)}(|\psi\rangle)=I^{(\ell, n)}(g|\psi\rangle)
$$

for all elements $g$ in $\Lambda_{\ell, n}$.

Question: But which such invariants are physically meaningful?

We begin to try to answer this question by noting that the only way to extract information from a quantum system is through quantum measurement. Thus, if we wish to extract information about quantum knot type from a quantum knot system $Q\left(\mathcal{K}^{(\ell, n)}, \Lambda_{\ell, n}\right)$, we of necessity must make a measurement with respect to some observable. But what kind of observable?

With this in mind, we will now describe quantum measurement from a different, but nonetheless equivalent perspective, than that which is usually given in standard texts on quantum mechanics. ${ }^{12}$ For knot theorists who might not be familiar with standard quantum measurement, we have included in the figure below a brief summary of quantum measurement. ${ }^{13}$

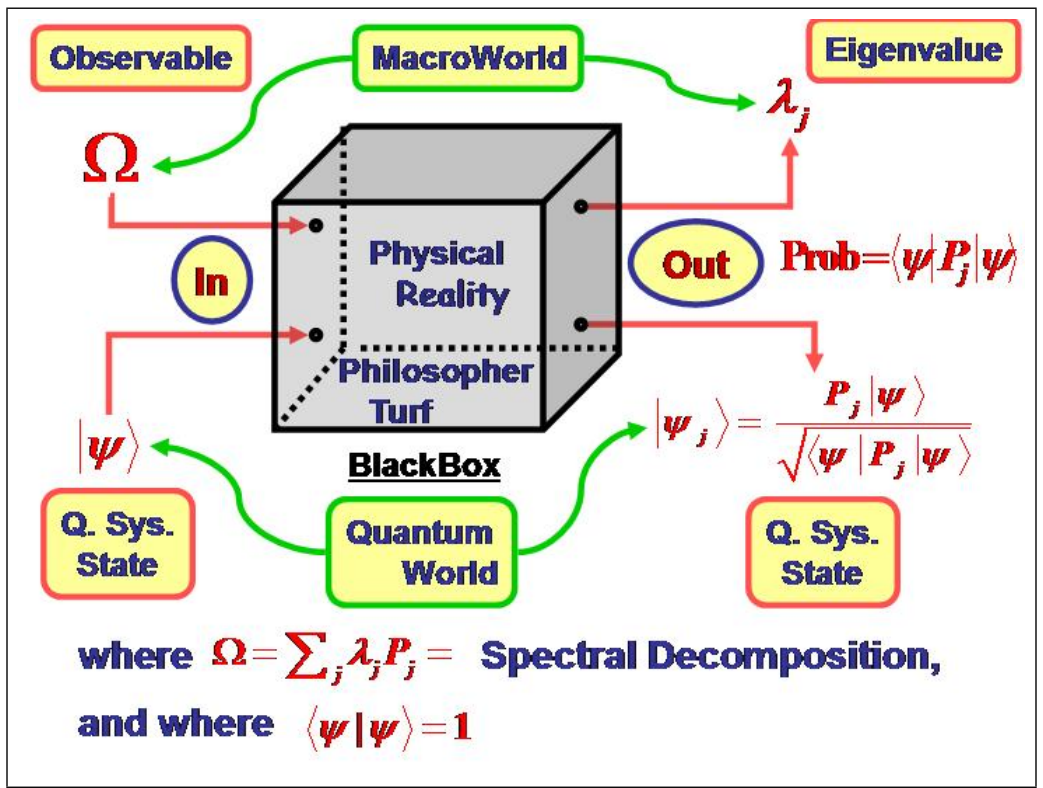

\footnotetext{
${ }^{12}$ In this paper, we will focus only on von Neumann quantum measurement. We will discuss more general POVM approach to quantum knot invariants in a later paper.

${ }^{13}$ For readers unfamiliar with quantum measurement, there are many references, for example, $[\mathbf{1 5}],[\mathbf{2 7}][\mathbf{3 7}],[39],[45]$, and $[47]$.
} 


\section{Von Neumann measurement.}

Let $\Omega$ be an observable for a quantum system $Q\left(\mathcal{K}^{(\ell, n)}, \Lambda_{\ell, n}\right)$, i.e., a Hermitian (self-adjoint) linear operator on the Hilbert space $\mathcal{K}^{(\ell, n)}$. Moreover, let

$$
\Omega=\sum_{j=1}^{m} \lambda_{j} P_{j}
$$

be the spectral decomposition of the observable $\Omega$, where $\lambda_{j}$ is the $j$-th eigenvalue of $\Omega$, and where $P_{j}$ is the corresponding projection operator for the associated eigenspace $V_{j}$.

Let $\mathcal{P}_{\Omega}^{(\ell, n)}$ denote the set of all probability distributions on the spectrum $\left\{\lambda_{1}, \lambda_{2}, \ldots, \lambda_{m}\right\}$ of $\Omega$, i.e.,

$$
\mathcal{P}_{\Omega}^{(\ell, n)}=\left\{p:\left\{\lambda_{1}, \lambda_{2}, \ldots, \lambda_{m}\right\} \longrightarrow[0,1]: \sum_{j=1}^{m} p\left(\lambda_{j}\right)=1\right\}
$$

We will call the probability distributions $p$ of $\mathcal{P}_{\Omega}^{(\ell, n)}$ stochastic sources.

Then each observable $\Omega$ uniquely determines a map

$$
\begin{aligned}
\widehat{\Omega}: \mathcal{K}^{(\ell, n)} & \longrightarrow \mathcal{P}_{\Omega}^{(\ell, n)} \\
|\psi\rangle & \longmapsto
\end{aligned}
$$

from quantum knots to stochastic sources on the spectrum of $\Omega$ given by

$$
p_{j}(|\psi\rangle)=\frac{\left\langle\psi\left|P_{j}\right| \psi\right\rangle}{\sqrt{\langle\psi \mid \psi\rangle}} .
$$

Thus, what is seen, when a quantum system $Q$ in state $|\psi\rangle$ is measured with respect to an observable $\Omega$, is a random sample from the stochastic source $\widehat{\Omega}(|\psi\rangle)$. But under what circumstances is such a random sample a quantum knot invariant?

Our answer to this question is that quantum knots $\left|\psi_{1}\right\rangle$ and $\left|\psi_{2}\right\rangle$ of the same knot $(\ell, n)$-type must produce random samples from the same stochastic source when measured with respect to the observable $\Omega$. This answer is captured by the following definition:

Definition 22.1. Let $Q\left(\mathcal{K}^{(\ell, n)}, \Lambda_{\ell, n}\right)$ be a quantum knot system, and let $\Omega$ be an observable on $\mathcal{K}^{(\ell, n)}$ with spectral decomposition

$$
\Omega=\sum_{j=1}^{m} \lambda_{j} P_{j}
$$

Then the observable $\Omega$ is said to be a quantum knot $(\ell, n)$-invariant provided

$$
\left\langle\psi\left|U P_{j} U^{-1}\right| \psi\right\rangle=\left\langle\psi\left|P_{j}\right| \psi\right\rangle
$$

for all quantum knots $|\psi\rangle \in \mathcal{K}^{(\ell, n)}$, for all $U \in \Lambda_{\ell, n}$, and for all projectors $P_{j}$. 
THEOREM 22.2. Let $Q\left(\mathcal{K}^{(\ell, n)}, \Lambda_{\ell, n}\right)$ and $\Omega$ be as given in the above definition. Then the following statements are equivalent:

1) The observable $\Omega$ is a quantum knot $(\ell, n)$-invariant

2) $\left[U, P_{j}\right]=0$ for all $U \in \Lambda_{\ell, n}$ and for all $P_{j}$.

3) $[U, \Omega]=0$ for all $U \in \Lambda_{\ell, n}$,

where $[A, B]$ denotes the commutator $A B-B A$ of operators $A$ and $B$.

The remaining half of this section is devoted to finding an answer to the following question:

Question: How do we find observables which are quantum knot invariants?

One answer to this question is the following theorem, which is an almost immediate consequence of the definition of a minimum invariant subspace of $\mathcal{K}^{(\ell, n)}$ :

THEOREM 22.3. Let $Q\left(\mathcal{K}^{(\ell, n)}, \Lambda_{\ell, n}\right)$ be a quantum knot system, and let

$$
\mathcal{K}^{(\ell, n)}=\bigoplus_{s} W_{s}
$$

be a decomposition of the representation

$$
\Lambda_{\ell, n} \times \mathcal{K}^{(\ell, n)} \longrightarrow \mathcal{K}^{(\ell, n)}
$$

into irreducible representations of the ambient group $\Lambda_{\ell, n}$. Then, for each $s$, the projection operator $P_{s}$ for the subspace $W_{s}$ is an observable which is a quantum knot $(\ell, n)$-invariant.

Yet another way of finding quantum knot invariants is given by the following theorem:

TheOREM 22.4. Let $Q\left(\mathcal{K}^{(\ell, n)}, \Lambda_{\ell, n}\right)$ be a quantum knot system, and let $\Omega$ be an observable on the Hilbert space $\mathcal{K}^{(\ell, n)}$. Let $S t(\Omega)$ be the stabilizer subgroup for $\Omega$, i.e.,

$$
S t(\Omega)=\left\{U \in \Lambda_{\ell, n}: U \Omega U^{-1}=\Omega\right\}
$$

Then the observable

$$
\sum_{U \in \Lambda_{\ell, n} / S t(\Omega)} U \Omega U^{-1}
$$

is a quantum knot $n$-invariant, where $\sum_{U \in \Lambda_{\ell, n} / S t(\Omega)} U \Omega U^{-1}$ denotes a sum over a complete set of coset representatives for the stabilizer subgroup $S t(\Omega)$ of the ambient group $\Lambda_{\ell, n}$.

ProOF. The observable $\sum_{g \in \Lambda_{\ell, n}} g \Omega g^{-1}$ is obviously an quantum knot $n$-invariant, since $g^{\prime}\left(\sum_{\left.g \in \Lambda_{\ell, n}\right)} g \Omega g^{-1}\right) g^{\prime-1}=\sum_{g \in \Lambda_{\ell, n}} g \Omega g^{-1}$ for all $g^{\prime} \in \Lambda_{\ell, n}$. If we let $|S t(\Omega)|$ denote the order of $|S t(\Omega)|$, and if we let $c_{1}, c_{2}, \ldots, c_{p}$ denote a complete set of coset representatives of the stabilizer subgroup $S t(\Omega)$, then $\sum_{j=1}^{p} c_{j} \Omega c_{j}^{-1}=$ $\frac{1}{|S t(\Omega)|} \sum_{g \in \Lambda_{\ell, n}} g \Omega g^{-1}$ is also a quantum knot invariant. 
Here are two other ways of creating observables which are quantum $(\ell, n)$-knot invariants:

- For each lattice knot $K \in \mathbb{K}^{(\ell, n)}$, we define the observable $P_{\Lambda_{\ell, n} K}$, called the orbit projector of $K$ as

$$
P_{\Lambda_{\ell, n} K}=\sum_{K^{\prime} \in \Lambda_{\ell, n} K}\left|K^{\prime}\right\rangle\left\langle K^{\prime}\right|
$$

- For each knot invariant $I: \mathbb{K} \longrightarrow \mathbb{C}$, we define the observable

$$
I^{(\ell, n)}=\sum_{K \in \mathbb{K}^{(\ell, n)}} I(K)|K\rangle\langle K|
$$

REMARK 22.5. We leave, as an exercise for the reader, the task of verifying that the subspace of $\mathcal{K}^{(\ell, n)}$ associated with the projection operator $P_{\Lambda_{\ell, n} K}$ is not an irreducible representation of the lattice ambient group $\Lambda_{\ell, n}$.

We end this section with an example of a quantum knot invariant:

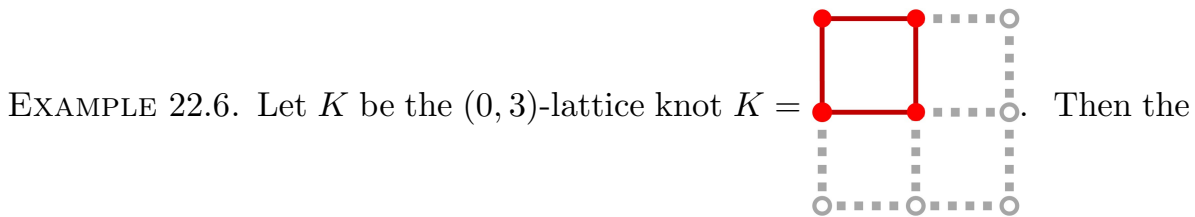
following observable $\Omega=P_{\Lambda_{0,3} K}$ is an example of a quantum knot (0,3)-invariant for the quantum knot system $Q\left(K^{(0,3)}, \Lambda_{0,3}\right)$ :

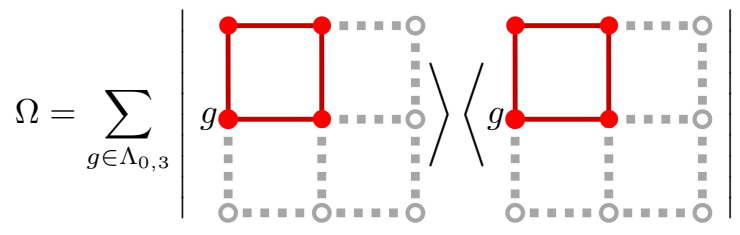

REMARK 22.7. For yet another approach to quantum knot measurement, we refer the reader to the brief discussion on quantum knot tomography found in item 11) in the conclusion of this paper.

\section{Conclusion: Open questions and future directions}

There are many possible open questions and future directions for research. We mention only a few. 
1) What is the exact structure of each of the lattice ambient groups $\Lambda_{\ell}$, $\widetilde{\Lambda}_{\ell}, \Lambda_{\ell, n}, \widetilde{\Lambda}_{\ell, n}$, and their direct limits? Can one write down an explicit presentation for for each of theses groups? for their direct limits? The fact that each of the lattice ambient groups is generated by involutions suggests that each may be a Coxeter group. Are the lattice ambient groups Coxeter groups? How are the lattice ambient groups related to the mosaic ambient groups $\mathbb{A}(n)$ found in $[\mathbf{2 5}]$. Are they in some since the same?

2) Unlike classical knots, quantum knots can exhibit the non-classical behavior of quantum superposition and quantum entanglement. Are topological entanglement and quantum entanglement related to one another? If so, how?

3) What other ways are there to distinguish quantum knots from classical knots?

4) Let $V_{K}(t)$ denote the Jones polynomial of lattice $(\ell, n)$-knots $K \in \mathbb{K}^{(\ell, n)}$. Then from Section 22 of this paper, we know that

$$
V^{(\ell, n)}(t)=\sum_{K \in \mathbb{K}^{(\ell, n)}} V_{K}(t)|K\rangle\langle K|
$$

is a family of quantum $(\ell, n)$-knot invariant observables parameterized by the parameter $t$. We will refer to this parameterized family $V^{(\ell, n)}(t)$ of observables simply as the Jones observable. Can we use the Jones observable to create an algorithmic improvement to the quantum algorithm given by Aharonov, Jones, and Landau in $[\mathbf{2}]$ ? (See also $[\mathbf{2 7}],[\mathbf{4 8}]$.)

5) How does one create quantum knot observables that represent other knot invariants such as, for example, the Vassiliev invariants?

6) What is gained by extending the definition of quantum knot observables to POVMs?

7) What is gained by extending the definition of quantum knots to mixed ensembles?

8) Define the lattice number of a knot $k$ as the smallest integer $n$ for which $k$ is representable as a lattice knot in the $n$-bounded lattice $\mathcal{L}_{0, n}$. In general, how does one compute the lattice number? Is the lattice number related to the crossing number of a knot? How does one find an observable for the lattice number?

9) Let $d(\ell, n)$ denote the dimension of the Hilbert space $\mathcal{K}^{(\ell, n)}$ of $(\ell, n)$-th order quantum knot. Find $d(\ell, n)$ for various values of $\ell$ an $n$. 
10) Consider the following alternate stronger definitions of order $(\ell, n)$ quantum knot type and quantum knot type:

Let $Q\left(\mathcal{K}^{(n)}, \Lambda_{\ell, n}\right)$ be a quantum knot system, and let $\mathcal{U}\left(\mathcal{K}^{(\ell, n)}\right)$ denote the Lie group of all unitary transformations on the Hilbert space $\mathcal{K}^{(\ell, n)}$. Define the continuous ambient group $\Lambda_{\ell, n}^{\text {cont }}$ as the smallest connected Lie subgroup of $\mathcal{U}\left(\mathcal{K}^{(\ell, n)}\right)$ containing the discrete ambient group $\Lambda_{\ell, n}$.

Proposition 7. Let $\mathbb{S}$ denote the set of wiggle, wag, and tug generators of the discrete ambient group $\Lambda_{\ell, n}$, and let $\lambda_{\ell, n}$ be the Lie algebra generated by the elements of the set

$$
\left\{\ln _{P}(g): g \in \mathbb{S}\right\},
$$

where $\ln _{P}$ denotes the principal branch of the natural log on $\mathcal{U}\left(\mathcal{K}^{(\ell, n)}\right)$. Then the continuous ambient group is given by

$$
\Lambda_{\ell, n}^{\text {cont }}=\exp \left(\lambda_{\ell, n}\right) .
$$

We define two quantum $(\ell, n)$-order quantum knots $\left|\psi_{1}\right\rangle$ and $\left|\psi_{2}\right\rangle$ to be of the same continuous knot $(\ell, n)$-type, written

$$
\left|\psi_{2}\right\rangle \underset{\ell}{\overparen{n}}\left|\psi_{2}\right\rangle
$$

provided there exists an element $g$ of the continuous ambient group $\Lambda_{\ell, n}^{\text {cont }}$ which transforms $\left|\psi_{1}\right\rangle$ into $\left|\psi_{2}\right\rangle$, i.e., such that $g\left|\psi_{1}\right\rangle=\left|\psi_{2}\right\rangle$. They are of the same continuous knot type, written $\left|\psi_{1}\right\rangle \asymp\left|\psi_{2}\right\rangle$, if there exist non-negative integers $\ell^{\prime}$ and $n^{\prime}$ such that $\iota^{\ell^{\prime}} \boldsymbol{Я}^{n^{\prime}}\left|\psi_{1}\right\rangle \underset{\ell+\ell^{\prime}}{\stackrel{n+n^{\prime}}{\asymp} \iota^{\ell^{\prime}}} \boldsymbol{Я}^{n^{\prime}}\left|\psi_{2}\right\rangle$.

Conjecture 2. Let $K_{1}$ and $K_{2}$ denote two $(\ell, n)$-order lattice knots, and let $\left|K_{1}\right\rangle$ and $\left|K_{2}\right\rangle$ denote the corresponding $(\ell, n)$-order lattice quantum knots. Then

$$
\left|K_{1}\right\rangle \underset{\ell}{\stackrel{n}{\ell}}\left|K_{2}\right\rangle \Longleftrightarrow K_{1} \underset{\ell}{\stackrel{n}{\sim}} K_{2} \quad \text { and } \quad\left|K_{1}\right\rangle \asymp\left|K_{2}\right\rangle \Longleftrightarrow K_{1} \sim K_{2}
$$

Thus, if this conjecture is true, these two stronger definitions of quantum knot $(\ell, n)$-type and quantum knot type fully capture all of classical tame knot theory. Moreover, these two stronger definitions have a number of advantages over the weaker definitions, two of which are the following:

- Under the Hamiltonians associated with the generators $\mathbb{S}$, the Schroedinger equation determines a connected continuous path in $\mathcal{K}^{(\ell, n)}$ consisting of $(\ell, n)$-th order quantum knots, all of the same quantum continuous knot $(\ell, n)$-type. 
- Although the following two quantum knots are not of the same discrete knot $(\ell, n)$-type, they are however of the same continuous knot $(\ell, n)$-type

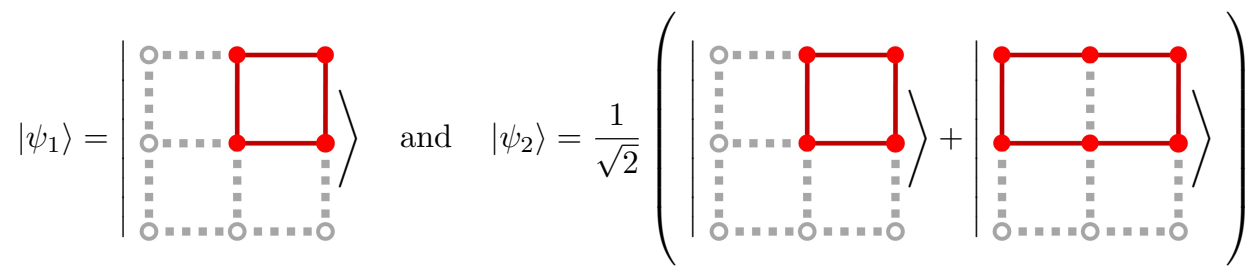

Is the above conjecture true? If so, what is the structure of the continuous

ambient group $\Lambda_{\ell, n}^{\text {cont }}$ ? What are its irreducible representations?

11) Can one create a more continuous definition of a quantum knot by quantizing the classical electromagnetic knots found in [26]? This question was the original motivation for this paper and for the paper [25].

12) Quantum knot tomography: Given repeated copies of an $(\ell, n)$-th order quantum knot $|\psi\rangle$, how does one employ the method of quantum state tomography[23] to determine $|\psi\rangle$ ? Most importantly, how can this be done with the greatest efficiency? For example, given repeated copies of the unknown quantum knot basis state

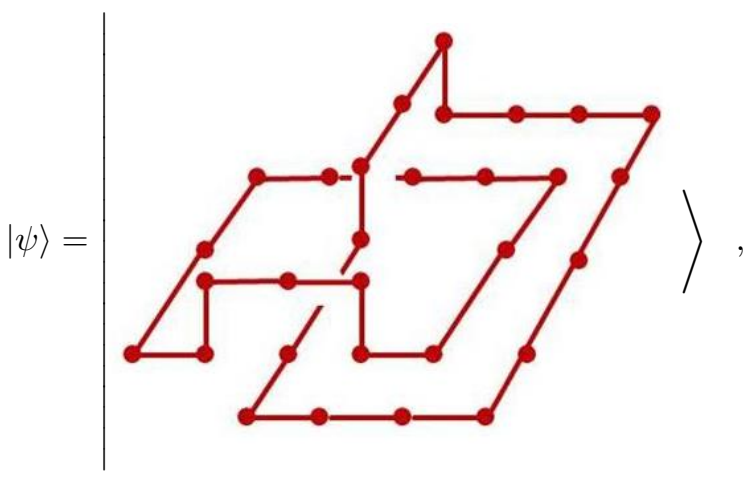

how does find a universal set of observables that is best for determining the quantum knot state in the sense of greatest efficiency for a given threshold $\epsilon ?$

13) Quantum Braids: One can also use lattices to define quantum braids. How is this related to the work found in $[\mathbf{1 7}],[\mathbf{2 2}],[\mathbf{4 6}]$ ?

14) Can quantum knot systems be used to model and to predict the behavior of

i) Quantum vortices in liquid Helium II? (See [41].)

ii) Quantum vortices in the Bose-Einstein condensate?

iii) Fractional charge quantification that is manifest in the fractional quantum Hall effect? (See [17] and [50].) 
In closing this section, we should finally also say that, in the open literature, the phrase "quantum knot" has many different meanings, and is sometimes a phrase that is used loosely. We mention only two examples. In [18], a quantum knot is essentially defined as an element of the Hilbert space with orthonormal basis in one-one-correspondence with knot types, rather than knot representatives. Within the context of the mosaic construction, a quantum knot in [18] corresponds to an element of the orbit Hilbert space $\mathcal{K}^{(n)} / \mathbb{A}(n)$. In $[\mathbf{6}]$ and in $[\mathbf{4 6}]$ the phrase "quantum knot" refers not to knots, but to the use of representations of the braid group to model the dynamic behavior of certain quantum systems. In this context, braids are used as a tool to model topological obstructions to quantum decoherence that are conjectured to exist within certain quantum systems.

\section{Appendix A: A quick review of knot theory}

In its most general form, knot theory is the study of the fundamental problem of placement:

The Placement Problem. When are two placements of a space $X$ in a space $Y$ the same or different?

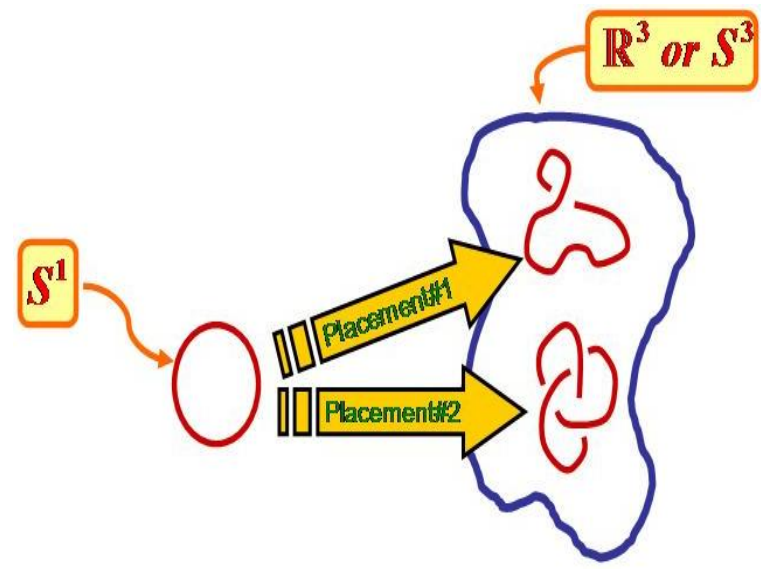

The placement problem.

In its most renowned form, knot theory is the study of the placement of a 1 -sphere ${ }^{14} S^{1}$ (or a disjoint union of 1 -spheres) in 3 -space $\mathbb{R}^{3}$ (or the 3 -sphere $S^{3}$ ), called the ambient space. In this case, "placement" usually means a smooth (or piecewise linear) embedding, i.e., a smooth homeomorphism into the ambient space. Such a placement is called a knot if a single 1-sphere is embedded ( or a link, if a disjoint union of many 1-spheres is embedded.)

Two knots (or links) are said to be the same, i.e., of the same knot type, if there exists an orientation preserving autohomeomorphism ${ }^{15}$ of the ambient space carrying one knot into the other. Otherwise, they are said to be different, i.e., of different knot type. Such knots are frequently represented by a knot diagram,

\footnotetext{
${ }^{14}$ By 1-sphere we mean a circle.

${ }^{15} \mathrm{~A}$ provenly equivalent definition is that two knots are of the same knot type if and only if there exists an isotopy of the ambient space that carries one knot onto the other.
} 
i.e., a planar 4-valent graph with vertices appropriately labelled as undercrossings/overcrossings, as shown in figure 2 .

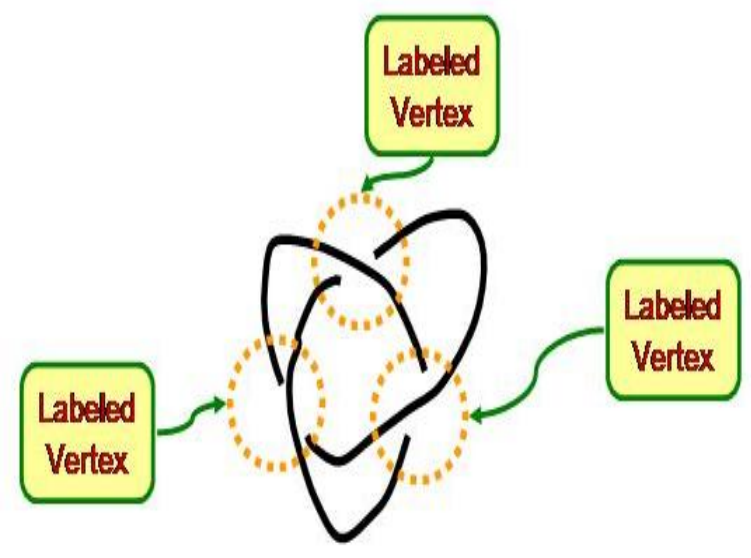

A knot diagram.

The fundamental problem of knot theory can now be stated as:

The Fundamental Problem of Knot Theory. When are two knots of the same or of different knot type?

A useful knot theoretic research tool is Reidemeister's theorem, which makes use of the Reidemeister moves as defined in figure 3 :

ThEOREM 24.1 (Reidemeister). Two knot diagrams represent the same knot type if and only if it is possible to transform one into the other by applying a finite sequence of Reidemeister moves.

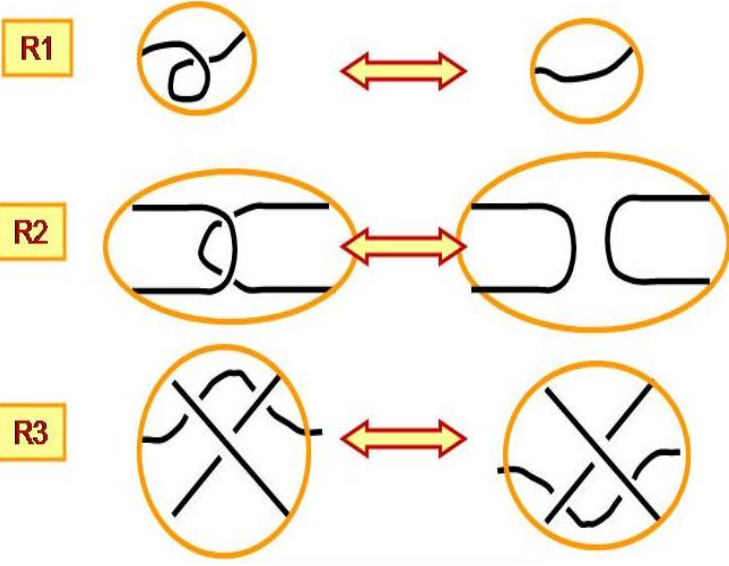

The Reidemeister moves. Th reader should note that these are local moves, as indicated by the local enclosure. 
The standard approach to attacking the fundamental problem of knot theory is to create knot invariants for distinquishing knots. By a knot invariant $I$ we mean a map from knots to a specified mathematical domain which maps knots (or links) of the same type to the same mathematical object. Thus, if an invariant is found to be different on two knots (or links), then the two knots (or links) cannot be of the same knot type!

For further information on knot theory, we refer the reader to, for example, $[1,4,7,12,21,24,35,40,42,43,44]$.

\section{Appendix B: A Rosetta Stone for notation}

This appendix gives a telegraphic summary of most of the mathematical symbols used in this paper.

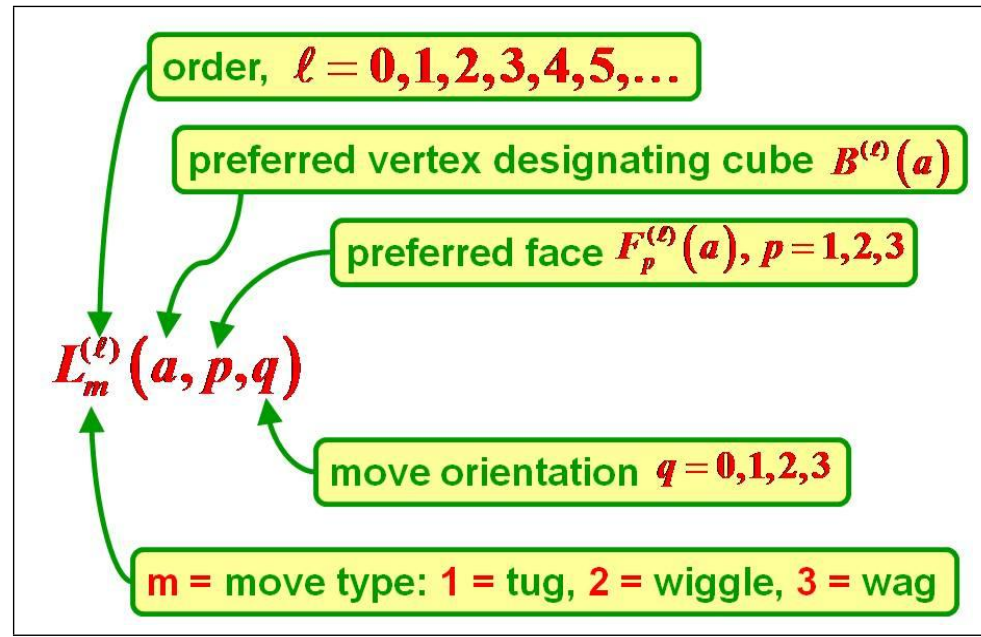

Summary of notation used to designate moves.

\begin{tabular}{|c|c||c||c|c|c|c|}
\hline & \multicolumn{1}{|c||}{$L_{m}(-,-, q)$} & & $L_{m}(-,-, 0)$ & $L_{m}(-,-, 1)$ & $L_{m}(-,-, 2)$ & $L_{m}(-,-, 3)$ \\
\hline \hline & & $m \backslash q$ & 0 & 1 & 2 & 3 \\
\hline \hline Tug & $L_{1}(-,-, q)$ & 1 & \lceil & $\square$ & $\sqsubset \mid$ & $\square$ \\
\hline Wiggle & $L_{2}(-,-, q)$ & 2 & \ulcorner & $\square$ & $\sqcap$ & $\square$ \\
\hline Wag & $L_{3}(-,-, q)$ & 3 & $\square$ & $\square$ & $\square$ & $\square$ \\
\hline
\end{tabular}

Summary of notation used to designate moves.

Please note that $L_{2}(-,-, 0)=L_{2}(-,-, 2)$ and $L_{2}(-,-, 1)=L_{2}(-,-, 3)$. 


$$
L_{m}(x, q)^{d x_{\lfloor p} d x_{p\rceil}}=\lim _{\ell \rightarrow \infty} L_{m}^{(\ell)}\left(\lfloor x\rfloor_{\ell}, p, q\right)
$$

where $d x_{\lfloor p} d x_{p\rceil}$ is the area 2 -form in 3 -space $\mathbb{R}^{3}$.

$a^{: 1^{2} \overline{2}^{3} 3}$ denotes the translate of the vertex $a$ given by

$$
a^{: 1^{2} \overline{2}^{3} 3}=a+2 \cdot 2^{\ell} e_{1}-3 \cdot 2^{-\ell} e_{2}+2^{-\ell} e^{3}
$$

where $e_{1}, e_{2}, e_{3}$ is the preferred orthonormal frame.

\section{Appendix C: The refinement morphism conjecture}

In this appendix, we give a constructive rationale for conjectures $1 \mathrm{~A}, 1 \mathrm{~B}$, and $1 \mathrm{C}$ of section 12 .

We begin by attempting to define $\boldsymbol{Я}(g)$ for each generator of the ambient group $\Lambda_{\ell}$, i.e., for each tug, wiggle, and wag in $\Lambda_{\ell}$. A possible definition is suggested by the figures given below:
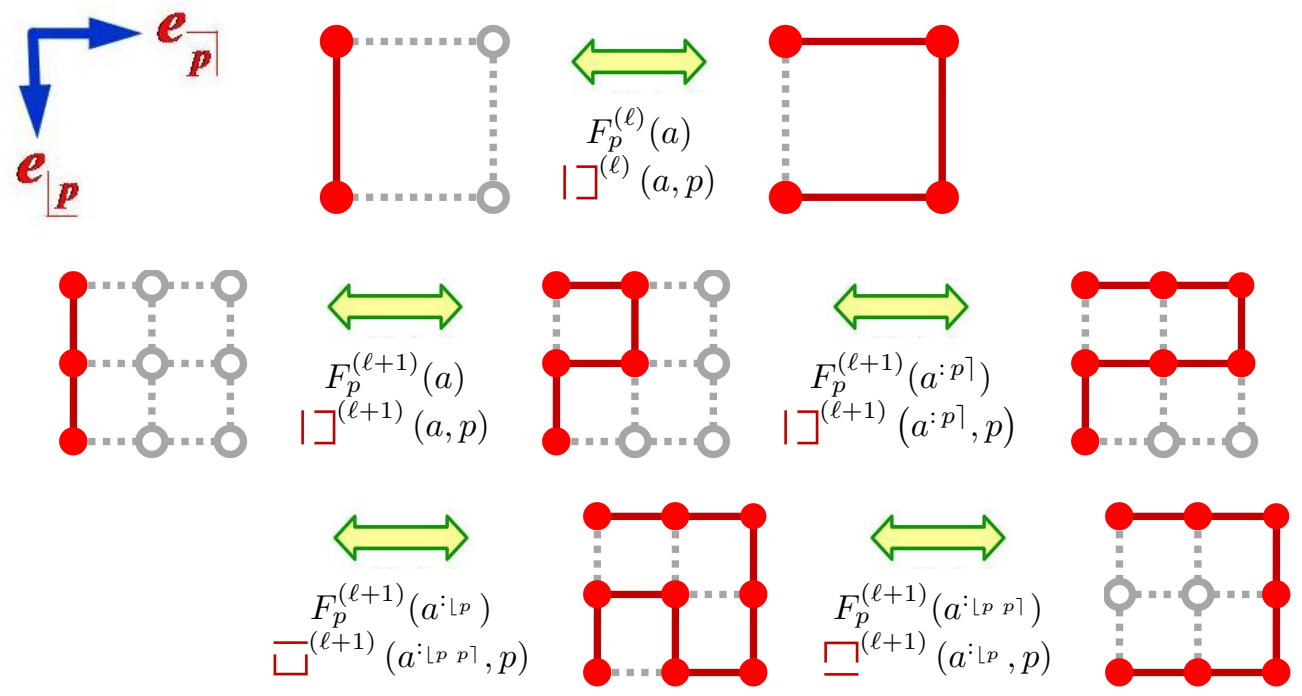

This is a free offprint provided to the author by the publisher. Copyright restrictions may apply. 

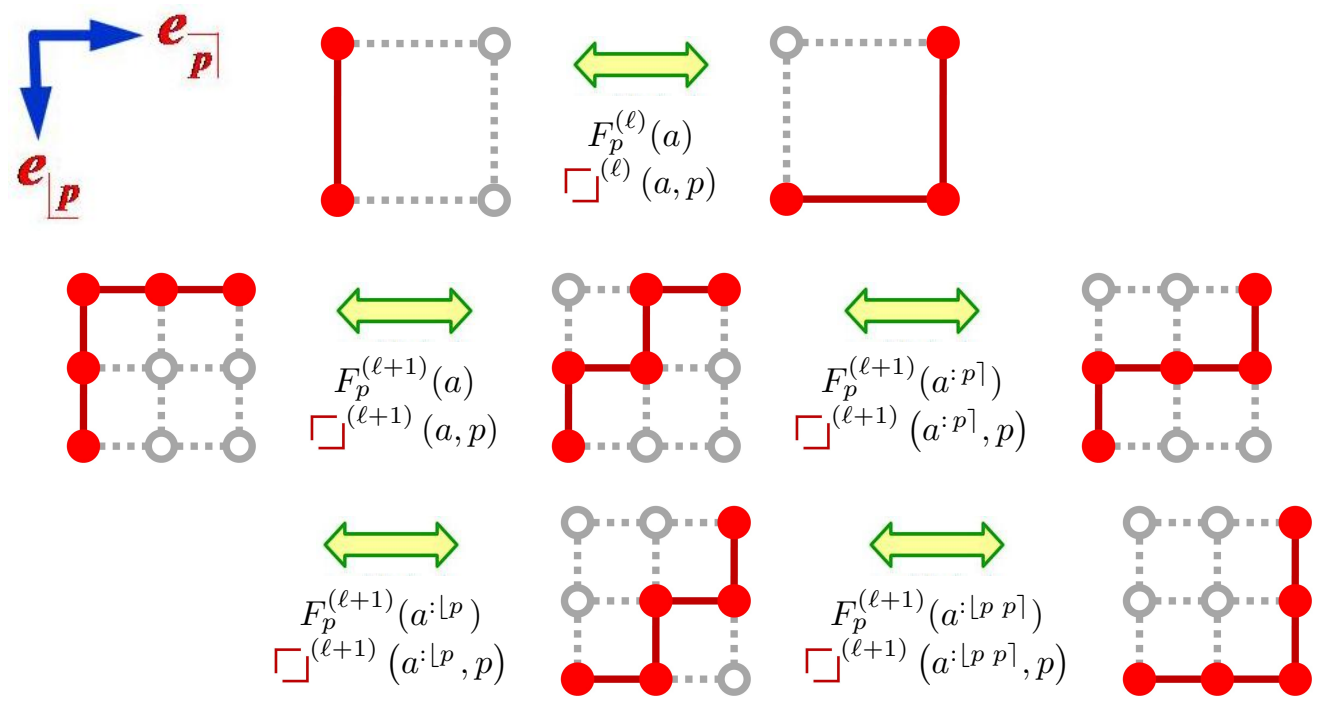

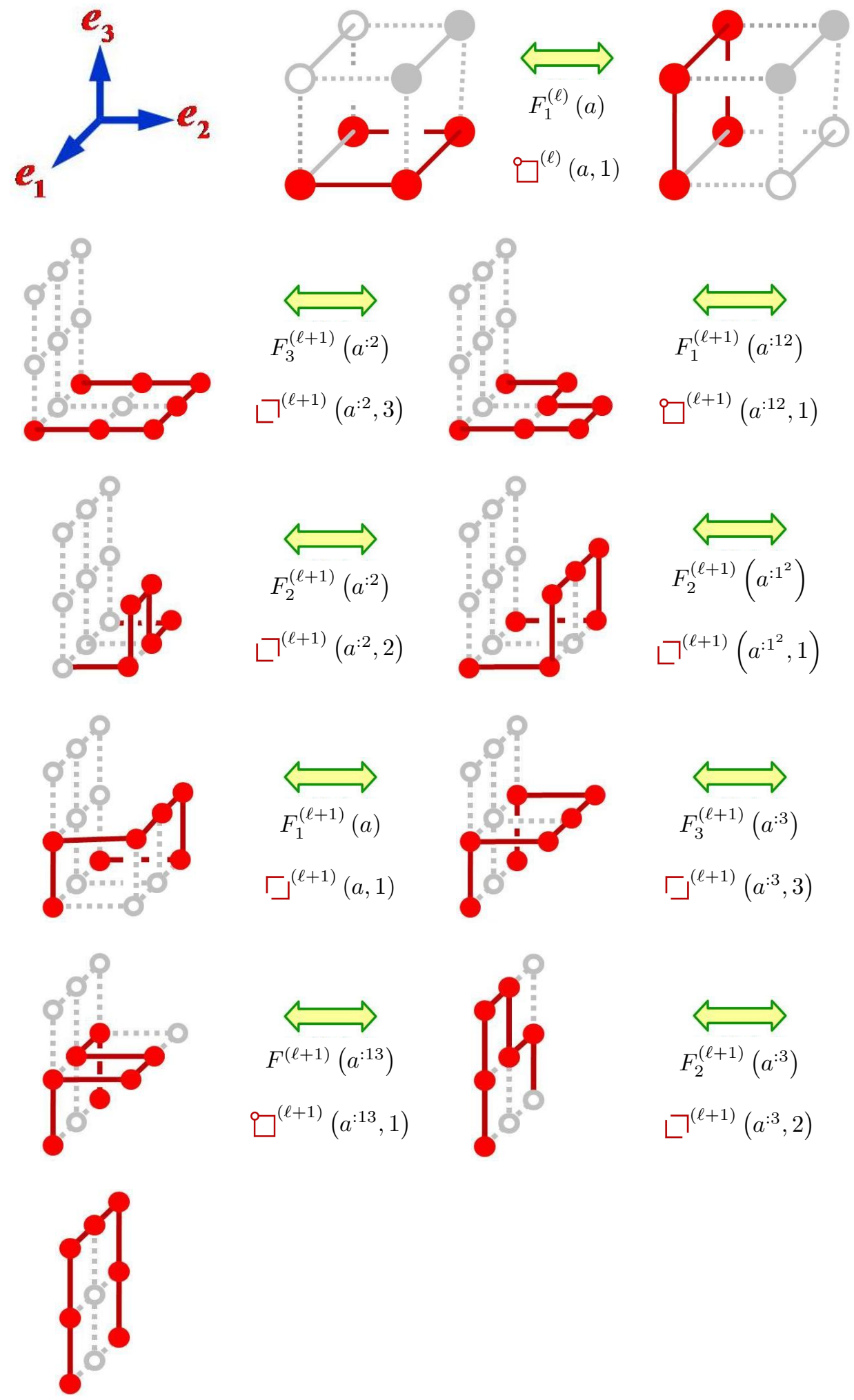

This is a free offprint provided to the author by the publisher. Copyright restrictions may apply. 
The definition suggested by the above figures is:

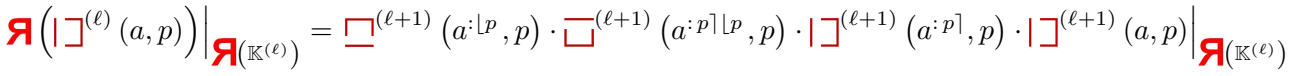

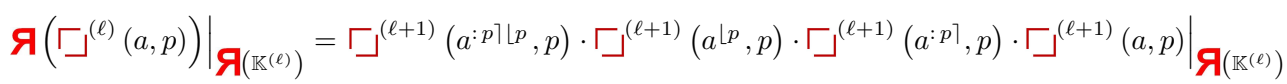

$$
\begin{aligned}
& \text { Я }\left.\left(\square^{(\ell)}(a, 1)\right)\right|_{\boldsymbol{Я}\left(\mathbb{K}^{(\ell)}\right)}=\bigsqcup^{(\ell+1)}\left(a^{: 3}, 2\right) \cdot \square^{(\ell+1)}\left(a^{: 13}, 1\right) \cdot \Gamma^{(\ell+1)}\left(a^{: 3}, 3\right) \cdot \Gamma^{(\ell+1)}(a, 1) \\
& \left.\cdot \square^{(\ell+1)}\left(a^{: 1^{2}}, 1\right) \cdot \square^{(\ell+1)}\left(a^{: 2}, 2\right) \cdot \square^{(\ell+1)}\left(a^{: 12}, 1\right) \cdot \square^{(\ell+1)}\left(a^{: 2}, 3\right)\right|_{\boldsymbol{Я}\left(\mathbb{K}^{(\ell)}\right)}
\end{aligned}
$$

where $\left.\boldsymbol{Я}\left(L_{*}^{(\ell)}(*, *, *)\right)\right|_{\boldsymbol{Я}\left(\mathbb{K}^{(\ell)}\right)}$ denotes a map $\boldsymbol{Я}\left(\mathbb{K}^{(\ell)}\right) \longrightarrow \boldsymbol{Я}\left(\mathbb{K}^{(\ell)}\right)$ from $\boldsymbol{Я}\left(\mathbb{K}^{(\ell)}\right)$ into itself.

We seek to construct a morphism $9: \mathbb{K}^{(\ell+1)} \longrightarrow \mathbb{K}^{(\ell+1)}$. Unfortunately, if for example we make the most straight forward definition by extending

$$
\left.\boldsymbol{Я}(\mid]^{(\ell)}(a, p)\right)\left.\right|_{\boldsymbol{Я}\left(\mathbb{K}^{(\ell)}\right)}: \boldsymbol{Я}\left(\mathbb{K}^{(\ell)}\right) \longrightarrow \boldsymbol{Я}\left(\mathbb{K}^{(\ell)}\right)
$$

to

$$
\boldsymbol{Я}\left(\left(\mid コ^{(\ell)}(a, p)\right)\right): \mathbb{K}^{(\ell+1)} \longrightarrow \mathbb{K}^{(\ell+1)}
$$

by defining

Я $\left(\mid \Xi^{(\ell)}(a, p)\right)=\underline{\square}^{(\ell+1)}\left(a^{:\lfloor p}, p\right) \cdot \square^{(\ell+1)}\left(a^{: p\rceil\lfloor p}, p\right) \cdot\left|\beth^{(\ell+1)}\left(a^{: p\urcorner}, p\right) \cdot\right| \beth^{(\ell+1)}(a, p)$, we produce an element of the ambient group $\Lambda_{\ell+1}$ which is not of order 2 . But $\mid]^{(\ell)}(a, p)$ is an element of $\Lambda_{\ell}$ of order 2 . Hence, the morphism $9: \Lambda_{\ell} \longrightarrow \Lambda_{\ell+1}$ we seek cannot be defined in this way! of

Fortunately, there are other possible elements of $\Lambda_{\ell+1}$ which are also extensions

$$
\left.\boldsymbol{Я}\left(\mid \beth^{(\ell)}(a, p)\right)\right|_{\boldsymbol{Я}\left(\mathbb{K}^{(\ell)}\right)}: \boldsymbol{Я}\left(\mathbb{K}^{(\ell)}\right) \longrightarrow \boldsymbol{Я}\left(\mathbb{K}^{(\ell)}\right) .
$$

We know from a previous theorem that each generator of $\Lambda_{\ell}$ is a product of disjoint transpositions. So a possible clue as to which extension to select comes from the following well known formula for transpositions:

$$
(1 n)=(((n-1) n) \wedge \ldots(\wedge(34) \wedge((23) \wedge(12)))),
$$

where $g \wedge h$ denotes $g \wedge h=g^{-1} h g$. This suggests the following approach to defining a morphism Я : $\Lambda_{\ell} \longrightarrow \Lambda_{\ell+1}$ : 
DEFINITION 26.1. We define the quandle product $\wedge$ as

$$
\begin{array}{ccc}
\Lambda_{\ell} \times \Lambda_{\ell} & \stackrel{\Lambda_{\ell}}{ } & \Lambda_{\ell} \\
\left(g_{1}, g_{2}\right) & \longmapsto & g_{1} g_{2}=g_{2}^{-1} g_{1} g_{2}
\end{array}
$$

Unfortunately, the quandle product " ${ }^{\wedge}$ ' is not an associative binary operation. To reduce the number and clutter of parentheses, we adopt a right to left precedence rule for parentheses. For example,

$g_{1} \wedge g_{2} \wedge g_{3}$ means $g_{1} \wedge\left(g_{2} \wedge g_{3}\right) \quad$ and $\quad g_{1} \wedge g_{2} \wedge g_{3} \wedge g_{4} \quad$ means $\quad g_{1} \wedge\left(g_{2} \wedge\left(g_{3} \wedge g_{4}\right)\right)$.

Equipped with the quandle product, we are now prepared to define the refinement map $9: \Lambda_{\ell} \longrightarrow \Lambda_{\ell+1}$ which we hope will be a morphism. We define the image of the generators as:

$$
\begin{aligned}
& \text { Я }\left(\mid \Xi^{(\ell)}(a, p)\right)=\underline{\square}^{(\ell+1)}\left(a^{:\lfloor p}, p\right) \wedge \varpi^{(\ell+1)}\left(a^{: p\rceil\lfloor p}, p\right) \wedge\left|\Xi^{(\ell+1)}\left(a^{: p\rceil}, p\right) \wedge\right| \Xi^{(\ell+1)}(a, p) \\
& \text { Я } \left.\left(\Gamma^{(\ell)}(a, p)\right)=\Gamma\right\lrcorner^{(\ell+1)}\left(a^{: p\urcorner\lfloor p}, p\right) \wedge \Gamma^{(\ell+1)}\left(a^{\lfloor p}, p\right) \wedge \Gamma^{(\ell+1)}\left(a^{: p\rceil}, p\right) \wedge \amalg^{(\ell+1)}(a, p) \\
& \text { Я }\left(\square^{(\ell)}(a, 1)\right)=\bigsqcup^{(\ell+1)}\left(a^{: 3}, 2\right) \wedge \square^{(\ell+1)}\left(a^{: 13}, 1\right) \wedge \Gamma \bigsqcup^{(\ell+1)}\left(a^{: 3}, 3\right) \wedge \coprod^{(\ell+1)}(a, 1) \\
& \wedge \square^{(\ell+1)}\left(a^{: 1^{2}}, 1\right) \wedge \square^{(\ell+1)}\left(a^{: 2}, 2\right) \wedge \square^{(\ell+1)}\left(a^{: 12}, 1\right) \wedge \square^{(\ell+1)}\left(a^{: 2}, 3\right)
\end{aligned}
$$

The key question is whether or not this extends to a group morphism 9 : $\Lambda_{\ell} \longrightarrow \Lambda_{\ell+1}$. For this to be true, each relation among the generators of $\Lambda_{\ell}$ must map to a relation among the generators of $\Lambda_{\ell+1}$.

Conjectures $1 \mathrm{~A}, 1 \mathrm{~B}$, and $1 \mathrm{C}$ of section 12 are essentially based on the above construction.

If the above conjecture is true, then we have created the following directed (lattice knot) ambient group system

$(\mathbb{K}, \Lambda)=\left(\mathbb{K}^{(0)}, \Lambda_{0}\right) \stackrel{\text { Я }}{\longrightarrow}\left(\mathbb{K}^{(1)}, \Lambda_{1}\right) \stackrel{\text { Я }}{\longrightarrow}\left(\mathbb{K}^{(2)}, \Lambda_{2}\right) \stackrel{\text { Я }}{\longrightarrow} \ldots \stackrel{\text { Я }}{\longrightarrow}\left(\mathbb{K}^{(\ell)}, \Lambda_{\ell}\right) \stackrel{\text { Я }}{\longrightarrow}\left(\mathbb{K}^{(\ell+1)}, \Lambda_{\ell+1}\right) \stackrel{\text { Я }}{\longrightarrow} \ldots$

Modulo this conjecture, the direct limit

$$
(\underset{\lim }{\longrightarrow} \mathbb{K}, \underset{\lim }{\longrightarrow} \Lambda)
$$

of this system exists. Thus, we can think of each element of $\lim \Lambda$, when restricted to a lattice knot $K \in \mathbb{K}$, as an ambient isotopy of $\mathbb{R}^{3}$ which respects the lattice knot $K$.

If we omit the tug moves in the above definition, we can, in like manner, define the inextensible (lattice knot ambient) group system 
$(\mathbb{K}, \widetilde{\Lambda})=\left(\mathbb{K}^{(0)}, \widetilde{\Lambda}_{0}\right) \stackrel{\text { Я }}{\longrightarrow}\left(\mathbb{K}^{(1)}, \widetilde{\Lambda}_{1}\right) \stackrel{\text { Я }}{\longrightarrow}\left(\mathbb{K}^{(2)}, \widetilde{\Lambda}_{2}\right) \stackrel{\text { Я }}{\longrightarrow} . \stackrel{\text { Я }}{\longrightarrow}\left(\mathbb{K}^{(\ell)}, \widetilde{\Lambda}_{\ell}\right) \stackrel{\text { Я }}{\longrightarrow}\left(\mathbb{K}^{(\ell+1)}, \widetilde{\Lambda}_{\ell+1}\right) \stackrel{\text { Я }}{\longrightarrow} \cdots$

In this case, each element of $\lim \widetilde{\Lambda}$, when restricted to a lattice $\operatorname{knot} K \in \mathbb{K}$, becomes an inextensible ambient isotopy.

\section{Appendix D: Oriented quantum knots}

We will now briefly outline how to define oriented quantum knots and oriented quantum knot systems. This will be accomplished by associating a qutrit (instead of a qubit) with each edge of the bounded cell complex $\mathcal{C}_{\ell, n}$.

Let ' $<$ ' denote the lex ordering of the set of edges $\mathcal{E}_{\ell, n}$ of the cell complex $\mathcal{C}_{\ell, n}$, as previously defined in Section 19 of this paper. Moreover, let $\mathcal{H}^{o}$ be the three dimensional Hilbert space with orthonormal basis

$$
\{|0\rangle=|\cdots \cdots\rangle,|1\rangle=|\longrightarrow\rangle=|\longrightarrow\rangle\} .
$$

Then the Hilbert space $\mathcal{G}_{\ell, n}^{o}$ of oriented lattice graphs of order $(\ell, n)$ is defined as the tensor product

$$
\mathcal{G}_{\ell, n}^{o}=\bigotimes_{E \in \mathcal{E}_{\ell, n}} \mathcal{H}^{o}
$$

where the tensor product is taken with respect to the above defined linear ordering ' $<$ '. Thus, as orthonormal basis for the Hilbert space $\mathcal{G}_{\ell, n}^{o}$, we have

$$
\left\{\underset{E \in \mathcal{E}_{\ell, n}}{\otimes}|c(E)\rangle: c \in \operatorname{Map}\left(\mathcal{E}_{\ell, n},\{0,1,2\}\right)\right\},
$$

where $\operatorname{Map}\left(\mathcal{E}_{\ell, n},\{0,1,2\}\right)$ is the set of all maps $c: \mathcal{E}_{\ell, n} \longrightarrow\{0,1,2\}$ from the set $\mathcal{E}_{\ell, n}$ of edges to the set $\{0,1,2\}$.

We identify in the obvious way each basis element

$$
\underset{E \in \mathcal{E}_{\ell, n}}{\otimes}|c(E)\rangle
$$

with a corresponding oriented lattice graph $G$. Under this identification, the space $\mathcal{G}_{\ell, n}^{o}$ becomes the Hilbert space with the orthonormal basis

$$
\left\{|G\rangle: G \text { an oriented lattice graph in } \mathcal{L}_{\ell, n}\right\} .
$$

We define the Hilbert space $\mathcal{K}_{o}^{(\ell, n)}$ of oriented lattice knots of order $(\ell, n)$ as the sub-Hilbert space of $\mathcal{G}_{\ell, n}^{o}$ with orthonormal basis

$$
\left\{|K\rangle: K \in \mathbb{K}_{o}^{(\ell, n)}\right\}
$$

where $\mathbb{K}_{o}^{(\ell, n)}$ denotes the finite set of oriented lattice knots of order $(\ell, n)$. We call each element of the Hilbert space $\mathcal{K}_{o}^{(\ell, n)}$ an oriented quantum knot.

For oriented lattice knots, oriented wiggles, wags, and tugs, and the corresponding oriented lattice graph ambient groups $\Lambda_{\ell, n}^{o}$ and $\widetilde{\Lambda}_{\ell, n}^{o}$ can be defined in the 
obvious manner. The remaining definitions and constructions are straight forward, and left to the reader.

REMARK 27.1. We should mention that, as abstract groups, the oriented lattice knot ambient groups $\Lambda_{\ell, n}^{o}$ and $\widetilde{\Lambda}_{\ell, n}^{o}$ are respectively isomorphic to the unoriented lattice knot ambient groups $\Lambda_{\ell, n}$ and $\widetilde{\Lambda}_{\ell, n}$.

\section{Appendix E: Quantum graphs}

We very briefly outline how to define quantum graphs and quantum graph systems.

Let $\mathcal{G}_{\ell, n}$ be the Hilbert space of lattice graphs with orthonormal basis

$$
\left\{|G\rangle: G \text { a lattice graph in } \mathcal{L}_{\ell, n}\right\},
$$

as defined in Section 19 of this paper.

We call each element of the Hilbert space $\mathcal{G}_{\ell, n}$ a quantum graph.

For lattice graphs, wiggles, wags, and tugs, and the corresponding lattice graph ambient groups $\Lambda_{\ell, n}^{\text {graph }}$ and $\widetilde{\Lambda}_{\ell, n}^{\text {graph }}$ can be defined in the obvious manner. The remaining definitions and constructions are straight forward, and left to the reader.

Question. Are the lattice graph lattice groups $\Lambda_{\ell, n}^{\text {graph }}$ and $\widetilde{\Lambda}_{\ell, n}^{\text {graph }}$ as abstract groups respectively isomorphic to the lattice knot ambient groups $\Lambda_{\ell, n}$ and $\widetilde{\Lambda}_{\ell, n}$ ?

\section{References}

[1] Adams, Colin C., "The Knot Book," W.H. Freeman, ((1994).

[2] Aharonov, D., Jones, V., Landau, Z., On the quantum algorithm for approximating the jones polynomial, http://arxiv.org/abs/quant-ph/0511096

[3] Bottema, O., and B. Roth, "Theoretical Knematics," Dover, (1990).

[4] Burde, Gerhard, and Heiner Zieschang, "Knots," Walter de Gruyter, (1985).

[5] Chen, G., Kauffman, L., Lomonaco, S.J. (eds.), "The Mathematics of Quantum Computation and Quantum Topology," Chapman \& Hall/CRC (2007)

[6] Collins, G.P., Computing with quantum knots, Sci. Am. April, 56-63 (2006)

[7] Crowell, R.H., Fox, R.H.: "Introduction to Knot Theory," Springer-Verlag, (1977)

[8] Dehn, M., P. Heegaard, Analysis Situs, in Encykl. Math. Wiss., vol. III, AB3, Leipzig, (1907), 153-220.

[9] Do Carmo, Manfredo P., "Differential Geometry of Curves and Surfaces," PrenticeHall, (1976).

[10] Flanders, Harley, "Differential Forms with Applications to the Physical Sciences," Academic Press, (1963).

[11] Fort, Hugo, Rodolfo Gambini, and George Pullin, Lattice knot theory and quantum gravity in loop representation, Vienna, Preprint, ESI 368 (1996). (Republished by Dover in 2008.)

[12] Fox, Ralph H., "A quick trip through knot theory," in "Topology of 3-Manifolds," (ed. by M.K. Fort, Jr.), Prentice-Hall, (1962), 120-167.

[13] Gauss, Karl Friedrich, Gauss 1833 Werke, Königlichen Gesellschaft der Wissenschaften zu Göttingen, (1877), 605- .

[14] Gelfand, I.M., and S.V. Fomin, "Calculus of Variations," Prentice-Hall, ((1963). 
[15] Helstrom, C.W., Quantum Detection and Estimation Theory, Academic Press, (1976)

[16] Hunt, K.H., "Kinematic Geometry of Mechanisms," Oxford Press, (1990).

[17] Jacak, L., Sitko, P.,Wieczorek, K.,Wójs, A., "Quantum Hall Systems: Braid groups, Composite Fermions, and Fractional Charge," Oxford University Press, (2003)

[18] Kauffman, L.H., Lomonaco, S.J., Quantum knots, Proc. SPIE, (2004). http://arxiv.org/abs/quant-ph/0403228

[19] Kauffman, L.H., Lomonaco, S.J., Jr., q-Deformed spin networks, knot polynomials, and anyonic topological quantum computation, J. Knot Theory 16(3), (2007), 267-332. http://xxx.lanl.gov/abs/quant-ph/0606114

[20] Kauffman, L.H., Lomonaco, S.J., Jr., Spin networks and anyonic topological computing, Proc. SPIE, 6244 (2006). http,//xxx.lanl.gov/abs/quant-ph/0603131

[21] Kauffman, L.H., "Knots and Physics," 3rd edn., World Scientific, (2001)

[22] Kitaev, A.Y., Fault-tolerant quantum computation by anyons. http://arxiv.org/abs/quant-ph/9707021

[23] Leonhardt, U., Measuring the Quantum State of Light, Cambridge University Press. (1997)

[24] Lickorish, W.B.R., "An Introduction to Knot Theory," Springer, (1997)

[25] Lomonaco, Samuel J., and Louis H. Kauffman, Quantum knots and mosaics, Journal of Quantum Information Processing, vol. 7, nos. 2-3, (2008), 85-115. http://arxiv.org/abs/0805.0339

[26] Lomonaco, S.J., "The modern legacies of Thomson's atomic vortex theory in classical electrodynamics," AMS PSAPM/51, Providence, RI, (1996), 145-166

[27] Lomonaco, S.J., Jr., and L.H. Kauffman, Topological quantum computing and the Jones polynomial, Proc. SPIE 6244 (2006). http://xxx.lanl.gov/abs/quant-ph/0605004

[28] Lomonaco, S.J., Jr., A Rosetta stone for quantum mechanics with an introduction to quantum computation, PSAPM, 58, AMS, Providence, RI, pp. 3-65 (2002)

[29] Lomonaco, S.J., Jr. (ed.), "Quantum computation," PSAPM, 58, American Mathematical Society, Providence, Rhode Island (2002)

[30] Lomonaco, S.J., Jr., Five dimensional knot theory, AMS CONM 20, (1984), 249-270. http://www.cs.umbc.edu/ ${ }^{\sim}$ lomonaco/ 5 knots $/ 5$ knots.pdf

[31] Lomonaco, S.J., Jr., The homotopy groups of knots I. How to compute the algebraic 3-type, Pacific J. Math. 95(2), (1981), 349-390

[32] Lomonaco, S.J., Jr., Inextensible knot theory, (in preparation).

[33] Madison, K.W., Chevy, F., Wohlleben, W., Dalibard, J., Vortex formation in a stirred Bose-Einstein condensate, Phys. Rev. Lett. 84, (2000), 806-809

[34] McCarthy, J.M., "An Introduction to Theoretical Kinematics," MIT Press, (1990).

[35] Murasugi, K., "Knot Theory and Its Applications," Birkhauser, (1996)

[36] O'Neill, Barrett, "Elementary Differential Geometry," (2nd edition), Academic Press, (1997).

[37] Nielsen, M.A., Chuang, I.L., "Quantum Computation and Quantum Information," Cambridge University Press, (2000)

[38] Paul, Richard P., "Robot Manipulators: Mathematics Programming ,and Control," M.I.T. Press, (1992).

[39] Peres, A., "Quantum Theory: Concepts and Methods," Kluwer (1993)

[40] Przytycki, Józef H., "Knots: From Combinatorics of Knot Diagrams to Combinatorial Topology Based on Knots," Cambridge University Press, (2010).

[41] Rasetti, M., and T. Regge, Vortices in He II, current algebras and quantum knots, Physica 80 A, North-Holland, (1975), 217-233

[42] Reidemeister, K., "Knotentheorie," Chelsea, (1948).

[43] Reidemeister, K., "Knot Theory," BCS Associates, Moscow, Idaho, ((1983). (Translation)

[44] Rolfsen, Dale, "Knots and Links," Publish or Perish, (1976).

[45] Sakuri J.J., "Modern Quantum Mechanics," revised edn., Addison-Wesley, (1994)

[46] Sarma, S.D., Freedman, M., Nayak, C., Topologically protected qubits from a possible non-Abelian fractional quantum hall state, Phys. Rev. Lett. 94, 166802-1-168802-4 (2005)

[47] Shankar, R., "Principles of Quantum Mechanics," 2nd edn., Plenum, (1994)

[48] Shor, P.W., Jordan, S.P., Estimating Jones polynomials is a complete problem for one clean qubit. http://arxiv.org/abs/0707.2831 
[49] Spivak, Michael, "A Comprehensive Introduction to Differential Geometry," vols. 1-5, (2nd edition), Publish or Perish, (1979).

[50] Wen, X.-G., "Quantum Field Theory of Many-Body Systems," Oxford Press, (2004)

[51] Zawitz, Richard E., United States Patent Number 4,509,929, April 4, 1985.

[52] Zee, A., "Quantum Field Theory in a Nutshell," Princeton University Press, (2003)

University of Maryland Baltimore County (UMBC), Baltimore, MD 21250 USA

E-mail address: lomonaco@umbc.edu

$U R L:$ http://www.csee.umbc.edu/ ${ }^{\sim}$ lomonaco

University of Illinois at Chicago, Chicago, IL 60607-7045 USA

E-mail address: kauffman@uic.edu

URL: http://www.math.uic.edu/ ${ }^{k}$ auffman 